
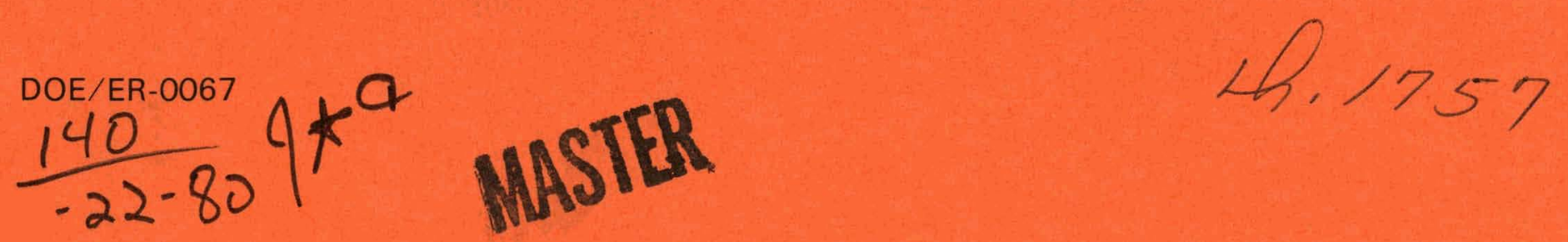

\title{
Report of the Subpanel on Accelerator Research and Development of the High Energy Physics Advisory Panel
}

June 1980

U.S. Department of Energy

Office of Energy Research

Division of High Energy Physics

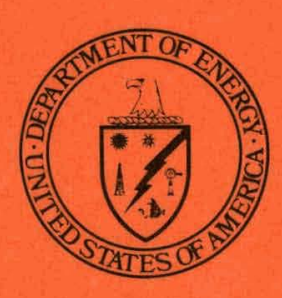




\section{DISCLAIMER}

This report was prepared as an account of work sponsored by an agency of the United States Government. Neither the United States Government nor any agency Thereof, nor any of their employees, makes any warranty, express or implied, or assumes any legal liability or responsibility for the accuracy, completeness, or usefulness of any information, apparatus, product, or process disclosed, or represents that its use would not infringe privately owned rights. Reference herein to any specific commercial product, process, or service by trade name, trademark, manufacturer, or otherwise does not necessarily constitute or imply its endorsement, recommendation, or favoring by the United States Government or any agency thereof. The views and opinions of authors expressed herein do not necessarily state or reflect those of the United States Government or any agency thereof. 


\section{DISCLAIMER}

Portions of this document may be illegible in electronic image products. Images are produced from the best available original document. 
Printed in the United States of America

$$
\text { Available from }
$$

National Technical Information Service U.S. Department of Commerce

5285 Port Royal Road

Springfie1d, VA 22161

NTIS price codes

Printed Copy:

9.00

Microfiche Copy: $\$ 3.50$ 


\section{Report of the Subpanel on Accelerator Research and Development of the High Energy Physics Advisory Panel}

June 1980

\section{U.S. Department of Energy}

\section{Office of Energy Research}

Division of High Energy Physics

Washington, D.C. 20545
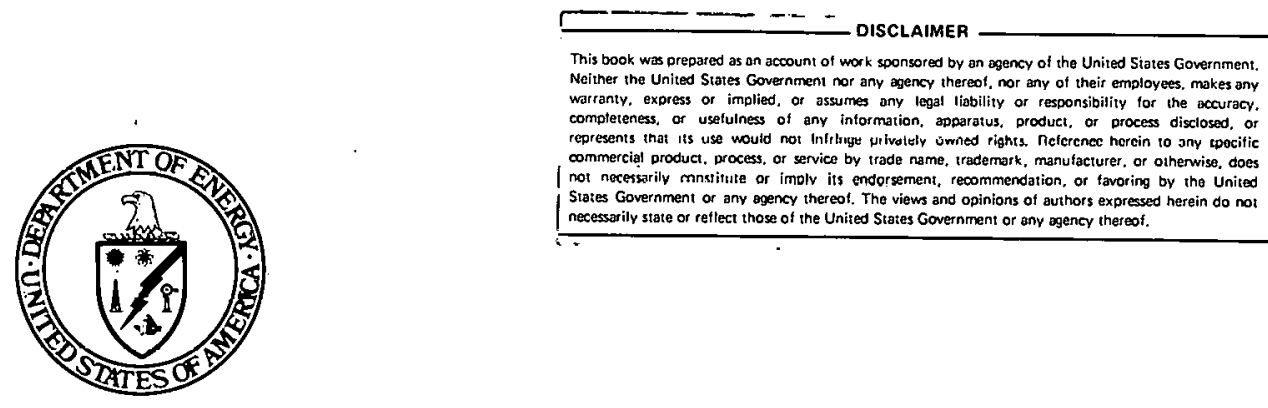


\section{Mail Address}

SLAC, P. O. Box 4349

Stanford, California 94305

June 4,1980

Dr. Edward A. Frieman, Director

Office of Energy Research

Department of Energy

Washington, D. C. 20545

Dear Ed:

I am pleased to transmit to you with unanimous endorsement by the High Energy Physics Advisory Panel this report on "Accelerator Research and Development in the United States High Energy Physics Program." This report, prepared by the HEPAP Subpanel on Accelerator R \& D under the Chairmanship of Professor M. Tigner, is a strong and persuasive call for the U.S. to put considerably more emphasis on long range accelerator R\&D.

We are currently devoting little more than $1 \%$ of the high energy physics budget to advanced accelerator $R \& D$ which focuses on technologies looking five or more years into the future. The central recommendation of this report is that this is inadequate, and that we should be prepared to increase this level substantially as the opportunities arise. A level of effort constituting up to $4 \%$ of the U.S. program, as advocated in this report, has our full endorsement.

This report is both an encyclopedia of very great value, of the ongoing efforts and of novel acceleration schemes under consideration, and a "call to arms" encouraging more attention on the part of the high energy community to advanced accelerator R\&D. As we see the end of the line for today' $s$ technology in advancing the high energy frontiers, it is of great importance that new techniques be explored and developed for the future. New accelerator techniques and the associated $R \& D$ programs will very likely also be of value to other fields of interest to the Department of Energy and the country.

I hope this excellent report will receive study and will be broadly distributed. I urge that its recommendations be used to guide policy for the accelerator $\mathrm{R} \& \mathrm{D}$ effort in our national high energy program.

Sincerely yours,

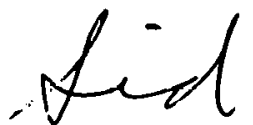

Sidney D. Drell

Chairman, HEPAP

dp

enclosure 
THIS PAGE

WAS INTENTIONALLY

LEFT BLANK 


\section{Cornell University}

Wilson Lab. Cornell Univ.

Ithaca, New York 14853

$607-256-4882$

August $26^{\circ}, 1980$

Prof. S. Drell

Chairman, HEPAP

Dear Sid:

Herewith I am transmitting the report of the HEPAP Subpanel on Accelerator R/D.

You will note that in the 50 odd years of American Accelerator science associated with particle physics research, enormous strides in increasing particle beam energies and in decreasing unit costs have been and are being made. nur report gives a catalog of high energy accelerator $R / D$ now in progress.

Our primary conclusion is that, despite the spectacular past and present accomplishments of the field, we must redouble our efforts to improve the cost effectiveness of our accelerators if the needs of US particle physics are to be met in the resource limited situation in which we find ourselves. This needed improvement will entail continual and vigorous improvement of existing technologies as well as the discovery and reduction to practice of new acceleration methods and technologies. If we are to meet this challenge we will need to devote more of our intellectual and monetary resources to high energy accelerator $R / D$ than is now our practice.

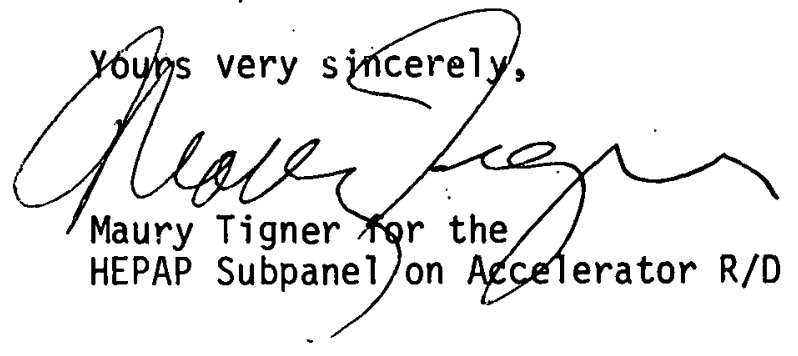

$\mathrm{MT} / \mathrm{mn}$

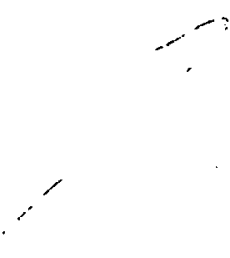


THIS PAGE

WAS INTENTIONALLY

LEFT BLANK 


\author{
Members of the Subpanel \\ on \\ Accelerator Research and Development \\ of the \\ High Energy Physics Advisory Panel
}

M. Tigner, Chairman

R. Diebold

K. Johnson

D. Keefe

A. McInturff

F. Mills

W. Panofsky

C. Pellegrini

J. Sandweiss

R. Schwitters

L. Teng

A. Tollestrup

W. Willis

M. Month

D. Sutter
Cornell University

ANL

BNL/CERN

LBL

BNL

FNAL

SLAC

BNL

Yale University

Harvard University

FNAL

FNAL

BNL/CERN

and

DOE

DOE 
THIS PAGE

WAS INTENTIONALLY

LEFT BLANK 
Accelerator Research and Development has always been an integral and important part of elementary particle physics research. The physics output has been paced, to a large extent, by the improvement in performance of our accelerators. In the past 50 years, equivalent beam energies available to experimenters have increased by a factor of ten million as a result of accelerator R\&D efforts. Not only has performance increased exponentially but unit costs have also been reduced significantly. Even in times of decreased real spending power for the field, significant energy increases were achieved.

Despite our remarkable achievements in unit cost reductions, these reductions have not kept pace with the energy increases achieved. This has resulted in a limitation in the number and diversity of high energy accelerator facilities available to workers in the field. Further decreases in diversity would be necessary to continue frontier research were the pace of cost reduction produced by new accelerator technologies not to increase. This would have a serious negative impact on the scientific productivity of U.S. elementary particle physics. The challenge is clear: we must redouble our efforts to reduce unit costs sharply while maintaining performance improvements through full exploitation of the potential of our current accelerator technologies and through invention of entirely new technologies and methods.

Historically, we have spent approximately 10 percent of our operating resources on accelerator R\&D activities. This includes both R\&D applied to projects (RDAP) and long-range R\&D (AARD) which is not associated with a specific project. Today this fraction for all accelerator R\&D is $14 \%$, reflecting our heavy commitment to superconducting magnet devel opment. The great majority of this is RDAP, as it needs to be. The Subpanel has determined that between $1 \%$ and $11 / 2 \%$ of operating resources are now devoted to AARU. If we are to meet the challenge of the future, more of our resources need to be devoted to this activity. We belleve that an appropriate level for long range R\&D would be about $4 \%$ of HEP operating resources.

It is obviuus that no level of expenditure for AARD can guarantee that the historical trend in unit cost reduction can continue. Nevertheless, the Subpanel is convinced that without increased effort on AARD significant unit cost reduction is most unlikely. Thus, we conclude that increased effort on AARD is an essential investment for the community at this time.

\section{Recommendation}

1. The high energy physics community should significantly increase its support for AARD. The Subpanel estimates, from an examination of significant areas of this research, that the level for AARD in the HEP accelerator field should be increased from about $1 \%$ up to about $4 \%$ of current operating resources. This increase should be achieved within the existing administrative framework. 
2. In reviewing accelerator R\&D, the Subpanel has identified certain specific technical areas that should be emphasized in AARD. This list is not presumed to be complete, exclusive, or to indicate relative priorities. Rather, it is intended to be an indication of the challenges of AARD and to underscore the importance of AARD to the field of high energy physics. This list of specific topics is:

(a) Development of very high field accelerator magnets and the evaluation of the practical limits of this technology. In view of the largescale of this enterprise and its uniqueness, the Subpanel recommends that this AARD effort be carried out as a collaborative effort among the laboratories having capability in this area. The Subpanel feels that this development of high-field magnets should be focused toward a specific accelerator goal.

(b) Development of liquid helium refrigerator systems with goals of improving efficiency and reliability and providing operation at reduced temperatures.

(c) Theoretical and experimental exploration of the limits to microwave linac gradients and to the peak powers that canl be delivered in the $S$ - to $X$-band regions.

(d) Basic physics and device development in superconducting RF accelerators.

(e) Theoretical and experimental studies of basic accelerator phenomena, particularly the beam-beam interaction and other performance-limiting phenomena.

(f) Search for and prel iminary development of new dccelerator schemes with high performance potential such as laser accelerators or other devices using ultra-high peak power with or without collective efforts.

(g) New techniques and devices for manipulating very high power and/or very high energy beams.

(h) The general problem of increasing the brightness of particle bcams with emphasis nn cooling high enteryy beams.

(i) Development of new beam diagnostic terchniqucs and Jevices.

3. Laboratory and University managements, aided by the agencies, should take specific measures to make participation in arseleraton R\&D practical and more desirable for high energy physics theorists and experimentalists not now so engaged. This could include payments of salary for sabbatical leaves and leaves of absence; and on-the-job training through seminars, summer schools and the use of specially assembled tutorial and review materials. Also included could be expansion of university-laburatory collaborations. 
4. Laboratory and University managements, aided by the agencies, should take specific measures to facilitate cross-fertilization from other fields such as Plasma Physics, Lasers and Materials Science, and other accelerator-related activities. This could include long-term visits by outstanding individuals in these fields for the purpose of working. directly with labortory staffs, giving tutorial seminars or lectures series, or participating in extended topical workshops. 
THIS PAGE

WAS INTENTIONALLY

LEFT BLANK 


\section{Contents}

\section{Page}

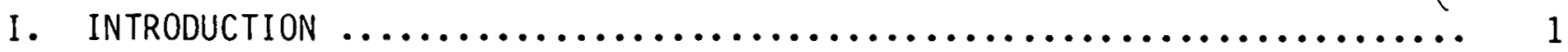

A. Historical Trends as a Guide to Future Needs $\ldots \ldots \ldots \ldots \ldots \ldots \ldots . . . .1$

B. Physics Expectations at Higher Energies $\ldots \ldots \ldots \ldots \ldots \ldots \ldots \ldots \ldots \ldots \ldots, 3$

1. Expected thresholds .................................. 3

2. Conjectured phenomena $\ldots \ldots \ldots \ldots \ldots \ldots \ldots \ldots \ldots \ldots \ldots \ldots \ldots, 9$

3. The unknown $\ldots \ldots \ldots \ldots \ldots \ldots \ldots \ldots \ldots \ldots \ldots \ldots \ldots \ldots, 10$

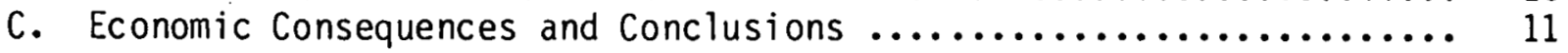

II. CHARGE TO THE hEPAP SUBPANEL ON ACCELERATOR R\&D $\ldots \ldots \ldots \ldots \ldots \ldots \ldots \ldots$

A. Charge to the HEPAP Subpanel on Accelerator R\&D and

Scope of Report ......................................... 13

B. Comments on the Charge ................................ 14

C. The Relationship of Accelerator R\&D to Detector R\&D ........... 15

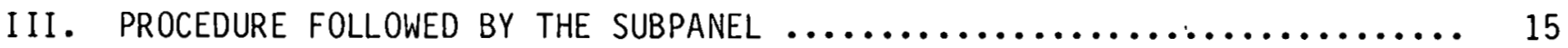

IV. STATUS OF HEP ACCELERATOR R\&D IN THE U.S. $\ldots \ldots \ldots \ldots \ldots \ldots \ldots \ldots \ldots, 17$

A. What is Accelerator R\&D? ............................... 17

B. Current Activities in Accelerator R\&D $\ldots \ldots \ldots \ldots \ldots \ldots \ldots \ldots \ldots, 18$

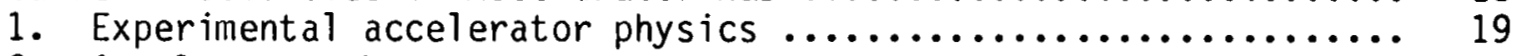

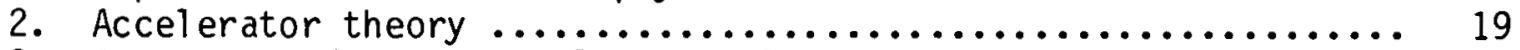

3. Systems studies of complete accelerators $\ldots \ldots \ldots \ldots \ldots \ldots \ldots \ldots, 20$

4. Superconducting magnets and cryogenics $\ldots \ldots \ldots \ldots \ldots \ldots \ldots \ldots, 21$

5. Superconducting RF cavities $\ldots \ldots \ldots \ldots \ldots \ldots \ldots \ldots \ldots \ldots \ldots \ldots, 21$

6. Normal conducting linac structures and cavities $\ldots \ldots \ldots \ldots \ldots .22$

7. High power RF and microwave sources $\ldots \ldots \ldots \ldots \ldots \ldots \ldots \ldots, 22$

8. Stochastic and electron cooling of beams $\ldots \ldots \ldots \ldots \ldots \ldots \ldots . .23$

9. Particle sources $\ldots \ldots \ldots \ldots \ldots \ldots \ldots \ldots \ldots \ldots \ldots \ldots \ldots \ldots, 24$

10. Beam transport $\ldots \ldots \ldots \ldots \ldots \ldots \ldots \ldots \ldots \ldots \ldots \ldots \ldots \ldots \ldots \ldots \ldots \ldots \ldots \ldots \ldots \ldots, 24$

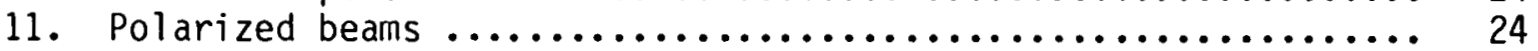

12. Control systems $\ldots \ldots \ldots \ldots \ldots \ldots \ldots \ldots \ldots \ldots \ldots \ldots \ldots \ldots \ldots \ldots, 25$

13. Non-traditional accelerators $\ldots \ldots \ldots \ldots \ldots \ldots \ldots \ldots \ldots \ldots, 25$

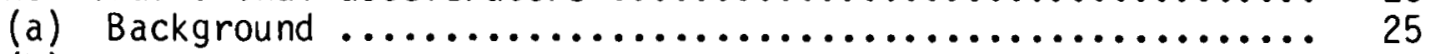

(b) Non-collective-effort accelerators for high currents..... 26

(c) Collective-effect ąccelerators $\ldots \ldots \ldots \ldots \ldots \ldots \ldots \ldots \ldots, 26$

(d) Power sources $\ldots \ldots \ldots \ldots \ldots \ldots \ldots \ldots \ldots \ldots \ldots \ldots \ldots \ldots \ldots \ldots, 27$

C. Resources Devoted to Accelerator R\&D $\ldots \ldots \ldots \ldots \ldots \ldots \ldots \ldots \ldots \ldots, 28$

D. Manpower in Accel erator R\&D ................................ 28

E. Resource Allocation for Accelerator R\&D $\ldots, \ldots, \ldots, \ldots, \ldots, \ldots, 35$ 


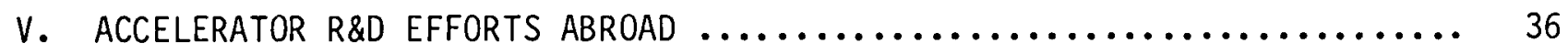

A. HEP Accelerator R\&D in Europe $\ldots \ldots \ldots \ldots \ldots \ldots \ldots \ldots \ldots \ldots \ldots \ldots, 36$

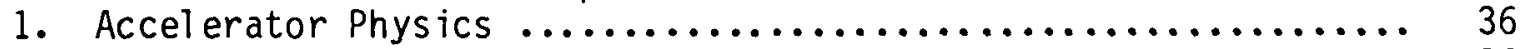

2. Advanced technology $\ldots \ldots \ldots \ldots \ldots \ldots \ldots \ldots \ldots \ldots \ldots \ldots \ldots \ldots \ldots,{ }_{36}$

(a) Superconducting magnets $\ldots \ldots \ldots \ldots \ldots \ldots \ldots \ldots \ldots \ldots \ldots, \quad 36$

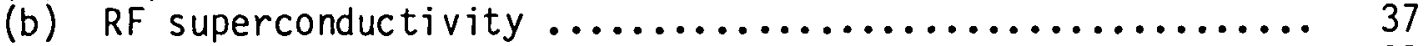

3. Project-oriented R\&D $\ldots \ldots \ldots \ldots \ldots \ldots \ldots \ldots \ldots \ldots \ldots \ldots \ldots \ldots \ldots \ldots \ldots, 37$

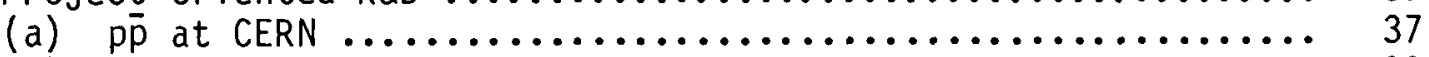

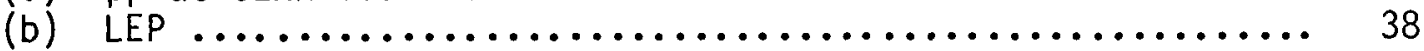

(c) HERA $\ldots \ldots \ldots \ldots \ldots \ldots \ldots \ldots \ldots \ldots \ldots \ldots \ldots \ldots \ldots,{ }_{3}$

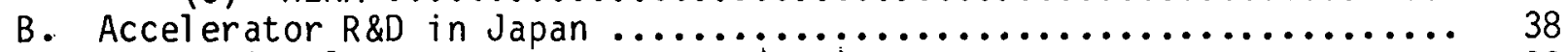

1. National Laboratory for HEP (KEK) ....................... 39

2. Institute for Nuclear Study, University or Tokyo ,.,......... 39

3. Superrennduct ivity work in industry ....................... 10

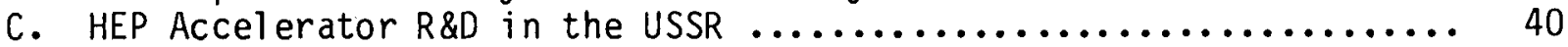

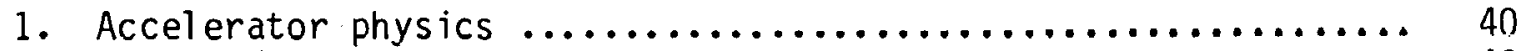

2. Advanced technology $\ldots \ldots \ldots \ldots \ldots \ldots \ldots \ldots \ldots \ldots \ldots \ldots \ldots, 40$

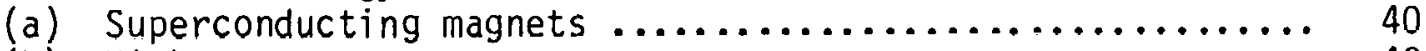

(b) High power sources $\ldots \ldots \ldots \ldots \ldots \ldots \ldots \ldots \ldots \ldots \ldots \ldots, 40$

(c) Ion sources, heavy ion accelerators, collective

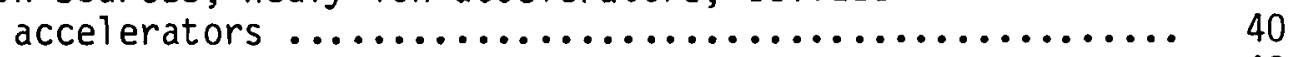

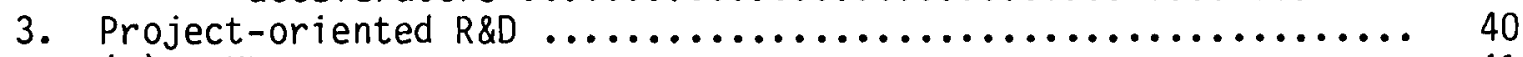

(a) UNK $\ldots \ldots \ldots \ldots \ldots \ldots \ldots \ldots \ldots \ldots \ldots \ldots \ldots \ldots \ldots \ldots \ldots \ldots \ldots \ldots \ldots, 41$

(b) Linear colliders $\ldots \ldots \ldots \ldots \ldots \ldots \ldots \ldots \ldots \ldots \ldots \ldots \ldots \ldots \ldots \ldots \ldots \ldots, 41$

VI. RELATIONSHIP OF U.S. ACCELERATOR R\&D TO EFFORTS ABROAD $\ldots \ldots \ldots \ldots \ldots, 42$

VII. CRITICAL REVIEW OF U.S. ACCELERATOR R\&D EFFORTS $\ldots \ldots \ldots \ldots \ldots \ldots \ldots \ldots . \ldots$

A. Challenges and Prospects $\ldots \ldots \ldots \ldots \ldots \ldots \ldots \ldots \ldots \ldots \ldots \ldots \ldots \ldots \ldots \ldots, 44$

B. Balance of Short-range, Mid-range and Long-range R\&D .......... 51

C. Technical Areas in Long-range R\&D Now Receiving

Insufficient Attention .............................. 51

1. Further refinements or extensions of established devices or technologies ............................ 51

2. Promising methods, devices and technologies $\ldots \ldots \ldots \ldots \ldots \ldots \ldots, 51$

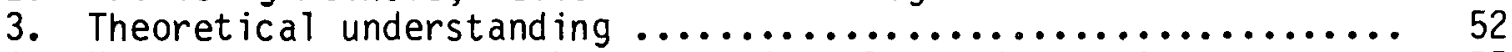

4. New or unexplored accelerator principles and techniques ...... 52

D. Communications Within the Field and With Other Fields $\ldots . . \ldots \ldots \ldots .53$

E. Manpower Resources and Training ........................... 54

F. Decision-making Processes and Resource Allocation .............. 54

VIII. CONCLUSIONS AND RECOMMENDATIONS $\ldots \ldots \ldots \ldots \ldots \ldots \ldots \ldots \ldots \ldots \ldots \ldots \ldots \ldots \ldots \ldots \ldots$

\section{APPENDICES}

I. R\&D on Accelerators to Produce Very High Beam Current ........... 61

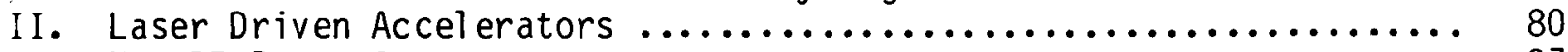

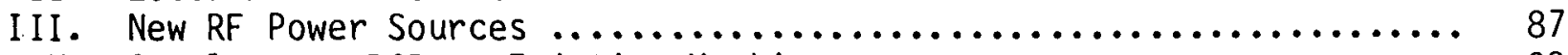

IV. Accel erator R\&D on Existing Machines $\ldots \ldots \ldots \ldots \ldots \ldots \ldots \ldots \ldots . .6 \%$ 


\section{INTRODUCTION}

In this report we review accelerator R\&D in the U.S. High Energy Physics (HEP) program. As a result of this study, we conclude that some shift in priority, particularly as regards long-range accelerator R\&D, is required to best serve the future needs of the U.S. HEP program. We also suggest some specific new directions for the U.S. R\&D effort.

Throughout this report, we shall use the term "accelerators" to denote not only devices which accelerate particles but also storage rings, colliders, etc.

Our assessments and recommendations are strongly influenced by the fifty year history of the field. Moreover, the particle energies needed to test the validity of current theoretical ideas reinforce our conviction that the historical thrust to higher energies must be maintained. Finally, we are persuaded.-that, as in the past, new and unsuspected phenomena will be uncovered at higher energies. These considerations lead us to the view that for the foreseeable future this thrust to higher energy must be maintained via a diversity of approaches: diversity of beam particles, accelerators, and colliders, as well as unorthodox approaches.

These needs have important economic consequences which present a direct and difficult challenge to the national accelerator R\&D program. Great ingenuity and resourcefulness will be required to meet this challenge.

\section{A. Historical Trends as a Guide to Future Needs}

The remarkable evolution of accelerator beam energies over the past five decades is partially displayed in the "Livingston Chart" of Figure 1. Beam energies (or fixed-target equivalent beam energies in the case of colliding beam accelerators) have risen by seven orders of magnitude in the past fifty years. It is noteworthy that this dramatic increase in collision energy has been achieved by a succession of diverse technologies: as any one technology reached saturation, it was superseded by a new, more powerful method based on novel ideas. This trend emphasizes the crucial importance of advanced accelerator $R \& D$, which is the source of such new approaches.

Much, but by no means all, progress in the understanding of elementary particle physics can be ascribed to increasing accelerator energies. In the 1930 's, elementary particle science had already established that the atom was a composite structure involving pointlike electrons bound electromagnetically to a heavy nucleus. The nucleus is itself a bound composite structure of particles, now recognized as protons and neutrons, or collectively as nucleons. The binding together of the nucleons in the nucleus is much strong than that of electrons to the nucleus. Disintegration of nuclei to study their structure therefore required projectile energies of several millions of electron volts (MeV) rather than the several thousands of electron volts (KeV) required to elucidate the electronic structure of the heavier atoms. As our ability to accelerate particles improved, we were able to see that the nucleons in turn are very 


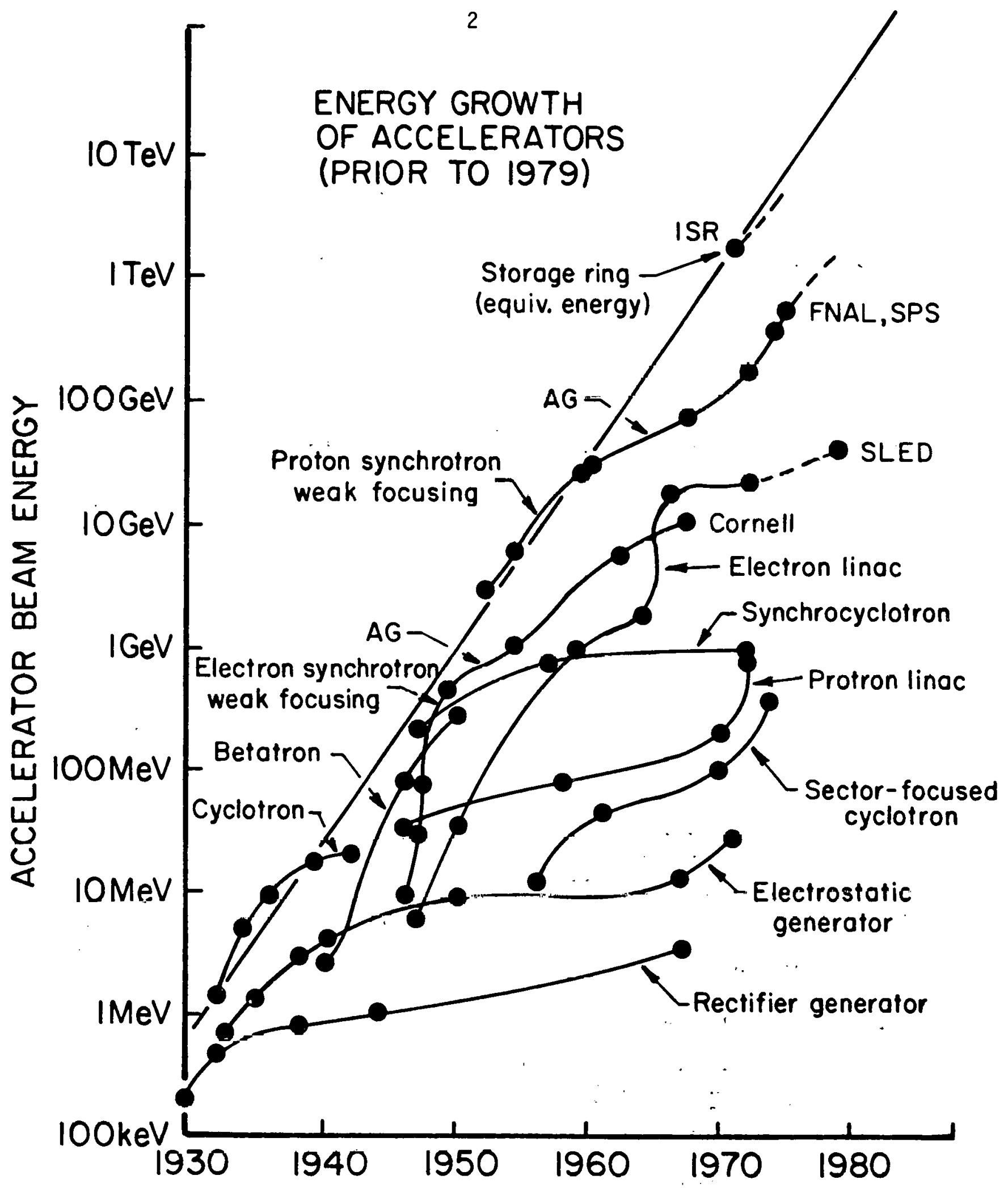

Fig. 1 Livingston Chart 
tightly bound composite particles with a rich substructure, the investigation of which has required projectiles of much higher energy. At the present time, available beam energies have reached into the hundreds ${ }^{\circ}$ of billions of electron volts (GeV). From experiments performed at these energies, a reasonably self-consistent picture of matter has emerged. Fundamental constituents of matter now appear to be pointlike leptons, of which the electron is one, and quarks which are new types of particles apparently confined in the nucleons.

Historically, as each new layer of matter was uncovered, a new "spectroscopy" was found which revealed the existence of smaller, more fundamental particles. Increasing volumes of data then led to a better understanding of the basic forces in nature. Yet as the basic richness of nature, on an ever-decreasing scale of distances, became apparent, so did its underlying simplicity: the laws of electricity and quantum mechanics were found to remain valid over an amazingly large range of distances. Moreover, during the past decade, experiments have shown the road to a basic unification of the diversity of forces in nature. It seems likely that the history of the past will repeat, and that higher energies will reveal further richness and new perspective in this fundamental picture. Indeed, the current theoretical understanding in the field provides a basis for specific expectations concerning new phenomena awaiting exploration with future machines at higher energies.

While these machines must clearly have sufficient energy to be above the threshold required for new effects, energy alone is not sufficient. We must also produce an intensity or "luminosity" which will allow an ohservahle rate for those fundamental processes which occur only very rarely. The event rate is given by the luminosity multipl led by the cross section (a quantity which measures the probability) for the reaction of interest. Figure 2 plots the collision energies and luminosities of several existing and planned accelerators and colliding beam devices. Also in the future are lines showing the luminosities required to yield sufficient intensity to permit investigation of all the various forces in nature. Such considerations provide an additional challenge to accelerator $R \& D$; the high energy machines of the future will only be useful if they yield adequate luminosity.

\section{B. Physics Expectations at Higher Energy}

In predicting the future utility of accelerators and colliding beam devices for research one has to be mindful of several basic factors:

(1) expected thresholds, (2) conjectured phenomena at ultra-high energies, and (3) "the unknown."

1. Expected Thresholds

of all the forces in nature, electromagnetism is the best understood. The unifying theory describing it, Quantum Electrodynamics (QED), has withstood experimental tests at the highest energies available. The electromagnetic force couples electric charges through the exchange 


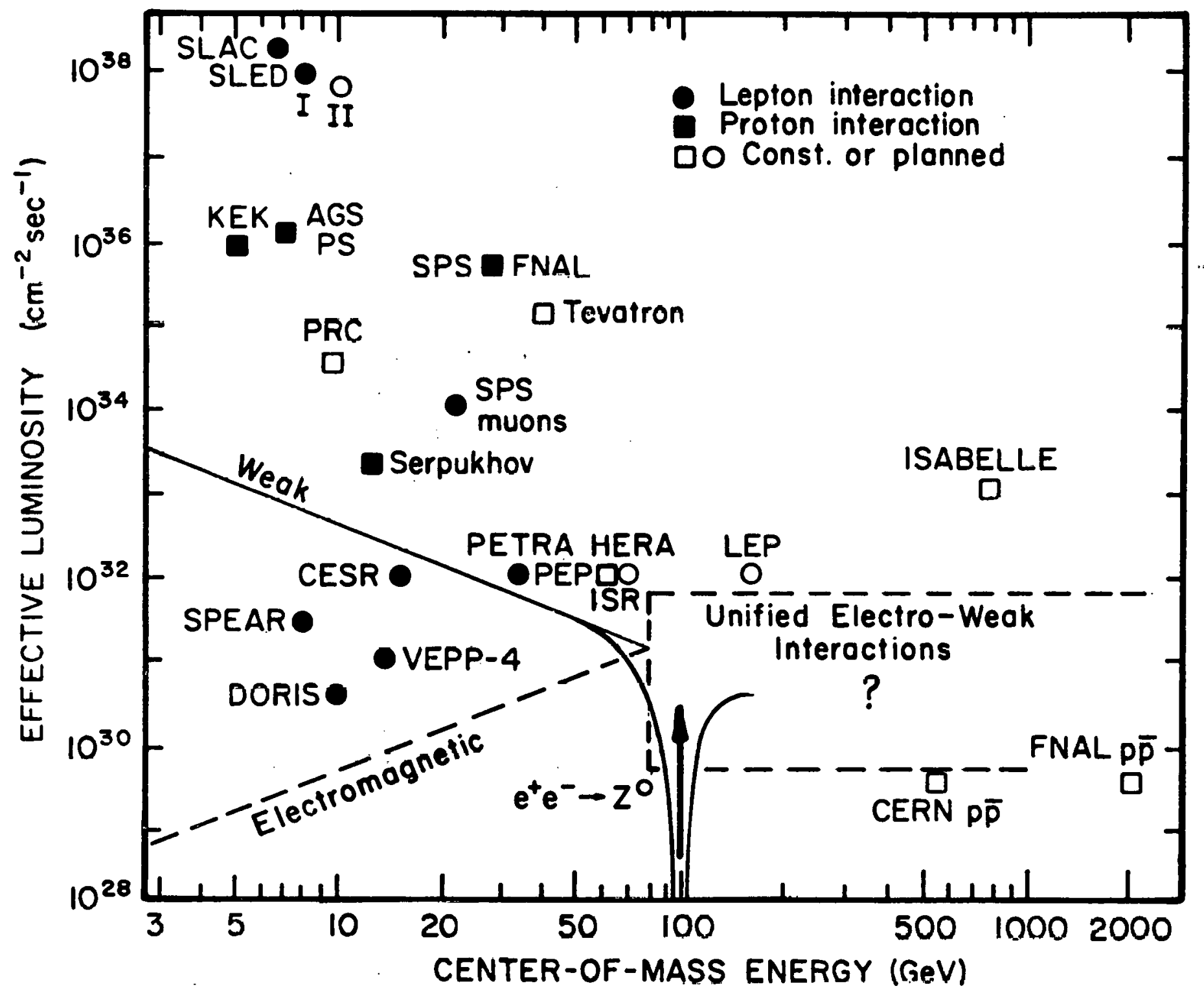

Effective luminosity vs. center-of-mass energy for the largest accelerators and storage rings now operating, in construction or planned. For accelerators, the target is assumed to be one meter of liquid hydrogen, except in the case of "SPS muons," where. 50 meters of $\mathrm{LH}_{2}$ is assumed.

The curves indicate the luminosities required to achieve a counting rate of one event per hour (assuming 100\% detection efficiency) for the electromagnetic and weak interactions. The resonant dip in the weak-interaction curve applies if the neutral-current weak interaction is mediated by a $Z^{\circ}$ boson of mass $100 \mathrm{GeV}$.

Fig. 2 
of massless vector particles called photons. Somewhat less understood is the weak interaction, which is bel ieved to couple the combinations of flavors of both ?eptons and quarks through exchange of massive internediate vector bosons. Finally, on a less detailed level of understanding, we believe the strongly interacting particles to be composed of quarks, which are thought to be confined within such particles (hadrons) by massless vector particles called gluons. These gluons couple to the quark property called color. The theory describing these interactions of quarks and gluons is called Quantum Chromodynamics (QCD).

This current picture, which is summarized in Table I, is expected to remain correct in most essential aspects but at present retains many unknowns.

$$
\because x
$$

The families of leptons and quarks which are known today are shown in Table II. Note that there is a missing quark (the $t$ ) whose mass, if it exists, may be above the reach of current machines. Note also that the discovery of these fermions originates from a large variety of sources. There never has been; $a$ and we surmise there never will be, a single "best" kind of machine.for discovery. Only further experiments, most requiring higher collision energy, are apt to clarify, modify and supplement this picture.

In accordance with this current description, collisions involving either electrons and positrons or nucleons and (anti) nucleons are described in terms of elementary lepton-lepton collisions or in terms of combinations of quark-quark or gqluon-gluon collisions, respectively. The reason is that hadron beams are a mixed collection of quarks, antiquarks ànd gluons with a wide energy spread, while lepton beams are accompanied by a small number of photons and intermediate vector bosons, increasing in numpers with energy. Table III roughly illustrates the situation for $\mathrm{e}^{+} \mathrm{e}^{-}$and $\bar{p} \mathrm{p}$ collisions.

According to this general picture is "expected that to explore adequately the threshold for a new phenomenon involving the creation of a new object of mass $M$, then the combined beam energies of mutually annihilating electrōn and positron beams should be, say, $10-20 \%$ above the value of that" mass; "while for proton colliding beam devices the combined beam energies might need to be as high as, say, roughly three times the value of the new 'mass. Happily, proton machines continue to be less expensive for a given beam energy than electron machines and thus compensate for:this difference in thresholds which is caused by the larger number of degrees of freedom in nucleonnucleon collisions: It also: follows that to explore a given kind of phenomenon the required luminosity for electron-positron machines should be roughly two orders of magnitude larger than that for protonantiproton devices. The reason for this is simply the disparate magnitudes of the structure constant of electrodynamics (1/137) and the coupling constant of QCD $(1 / 6)$. 
The Present Picture of the Interactions of Elementary Particles

\begin{tabular}{|c|c|c|c|}
\hline & Electromagnetic & Weak & Strong \\
\hline $\begin{array}{l}\text { Couples } \\
\text { with }\end{array}$ & Electric charge & flavor & color \\
\hline $\begin{array}{l}\text { Carried by } \\
\text { termions }\end{array}$ & $\begin{array}{l}\text { quarks and } \\
\text { leptons }\end{array}$ & $\begin{array}{l}\text { quarks and } \\
\text { leptons }\end{array}$ & q!ark.s \\
\hline $\begin{array}{l}\text { Couples } \\
\text { through } \\
\text { exchange of }\end{array}$ & $\begin{array}{c}\text { photons } \\
\text { (neutral, massless) }\end{array}$ & $\begin{array}{l}\text { intermediate } \\
\text { vector bosons } \\
\text { (neutral \& } \\
\text { charged, } \\
\text { massive) }\end{array}$ & $\begin{array}{c}\text { gluons } \\
\text { (colored \& } \\
\text { massless) }\end{array}$ \\
\hline
\end{tabular}




\begin{tabular}{|c|c|c|c|c|c|}
\hline & \multicolumn{2}{|c|}{ LEPTONS } & \multicolumn{2}{|c|}{ QUARKS } & \\
\hline $\begin{array}{l}\text { ELECTRIC } \\
\text { CHARGE }\end{array}$ & -1 & 0 & $-\frac{1}{3}$ & $+\frac{2}{3}$ & DATES \& NOTES \\
\hline & $e^{-}$ & $v_{e}$ & $d$ & $u$ & $\begin{array}{l}\leq 1935 \text { Easic building blocks of all } \\
\text { chemical elements }\end{array}$ \\
\hline & $\mu^{-}$ & $\nu_{\mu}$ & $s$ & c & $\begin{array}{l}1936 \text { Muon }(\mu) \text { discovered in cosmic rays } \\
1947 \text { Strange particles } \\
1974-76 \mathrm{\psi} / \mathrm{J} \text {, Charm }\end{array}$ \\
\hline & $\tau^{-}$ & $v_{\tau}$ & $b$ & $t(?)$ & $\begin{array}{l}\text { 1975-77 } \tau \text { discovered in } e-\mu \text { events } \\
1977 \text { Lpsilon }(T) \text { discovered }\end{array}$ \\
\hline
\end{tabular}

Table II - Three generations of leptons and quarks 
Comparison of $e^{+} e^{-}$and $p \bar{p}$ collisions

$e^{+} e^{-}$Collisions

$p \bar{p}$ Collisions

$\mathrm{e}^{+} \mathrm{e}^{-}$makes new charged quanta through photons or intermediate vector bosons

q⿳亠 or ḡ make new colored quanta

Bhabha

scattering
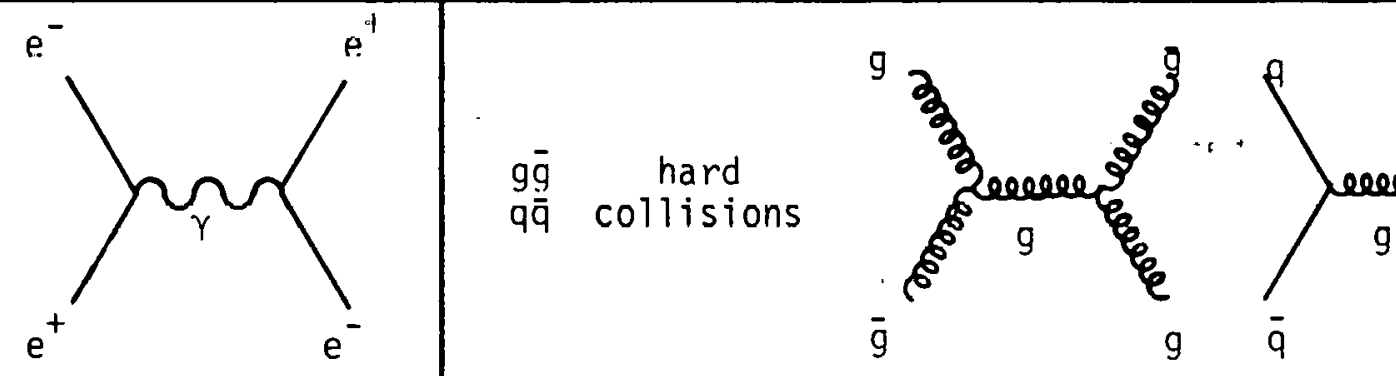

$\mathrm{g}$

Strength: $\alpha^{2}=(1 / 137)^{2}$

Strength: $\quad \alpha_{S}^{2} 2(1 / 6)^{2}$

Annihilation

into charged

fermions $F$

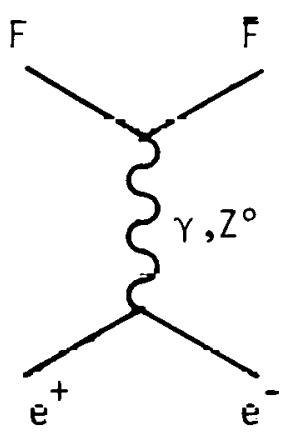

g⿳亠 hard

$q \bar{q}$ collisions

$\bar{g}$

Annihilation

into colored

ferminns $F$

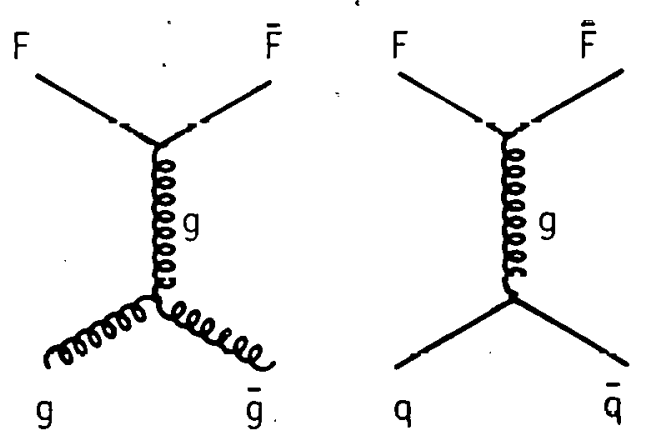

Threshold: $\mathrm{m}_{\mathrm{F}} \hat{\imath} 0.8 \mathrm{E}_{\text {beam }}$

Threshold: $\begin{aligned} \mathrm{m}_{\mathrm{F}} & \stackrel{\varsigma}{\imath} 0.8 \mathrm{Eg}, \mathrm{q} \\ & \lesssim 0.4 \mathrm{E}_{\text {proton }}\end{aligned}$

Table III. 
One must, of course, take account of the fact that in electronpositron collisions we are starting from a cleaner initial state and that therefore the interpretability of the final event will generally be enhanced. Also, electrons and positrons open the possibility of direct channel resonances leading, for instance, to copious production of such objects as the $Z^{0}$ or other new particles. Some new discoveries will therefore be made easier by the extension of electron-positron energies. However, such considerations will ultimately be dwarfed by the technological opportunities to reach very high energies to start with, and these technological opportunities look most promising for protons. In view of the above, electron-positron and proton-(anti) proton collisions offer alternative paths to particle discoveries at highest energies: electron-positron collisions can lead to discoveries less masked by background, while proton collisions will ultimately reach higher threshold energies and offer the possibility to explore channels not accessible through electron-positron collisions.

According to the above theoretical picture, the next families of fundamental objects that might be uncovered are the intermediate vector bosons in their neutral and charged varieties. This will require collision energies approaching $100 \mathrm{GeV}$ for electron-positron collisions and several times that for hadron collisions. These particles should be produced by machines to be built in the late $1980^{\prime} \mathrm{s}$. It is expected that production of the $Z^{0}$ should be sufficiently copious that electron-positron colliders will be "factories" of such objects.

\section{Conjectured Phenomena}

According to current thought, the above picture cannot be complete and may well be defective. Since the vector carriers of the weak interactions have finite mass, they cannot be purely transverse in character as is the photon and its electromagnetic field, there should also exist scalar particles related to the longitudinal components. While specific predictions of the nature and masses of these "Higgs"

particles remain uncertain, they might constitute a whole new family of objects; and these in turn might give rise to a new spectroscopy.

We also do not know whether the entries in Table II (assuming that the $t$ quark will be discovered) are complete. There could be further generations of leptons and quarks at higher energies, and their discovery would have profound implications for both basic particle theory and cosmology. If the $t$ quark does not exist, considerable re-thinking and rearrangement of the quark pattern will be needed.

Beyond the lepton and quark generations described above, there might be entire new families of fermions which do not interact through the color forces, like quarks, but which have carriers of a new type. Some of this might be visible in the $1 \mathrm{TeV}$ center-of-mass energy regions. Moreover, there is an excellent rationale for going beyond 
that. For one thing, one must recognize that the variation of basic cross sections with energy predicted by QCD is largely logarithmic, and that therefore considerable increments in collision energies are necessary in order to pin down such basic dependencies reliably.

There are indications from cosmic-ray experiments that at beam momenta against fixed targets in excess of, perhaps, $10^{14} \mathrm{eV} / \mathrm{c}$ new phenomena come into play, which seem to give rise to jets of very high multiplicity of charged particles but apparently free from neutral pions. These phenomena, to be seen at all in cosmic rays, must have cross sections of sufficient magnitude to make them accessible to future colliders even at luminosities well below those attained in the past. Well above even these energies, there is the conjecture that some extremely heavy objects exist in nature which become dominant once the grand unification of electromagnetic, weaki and strong interactions has taken place. These particles have masses well in excess of those conceivably attainable by man-made accelerators in the foreseeable future.

Thus it is indeed unlikely that the quest for higher and higher energies will become unproductive, i.e., will lead to a "flat" world of the energy dependence of elementary phenomena. Rather, the manifestation of such high masses will impact observable phenomena progressively more significantly as collision energies become higher.

3. "The Unknown"

This discussion is, of course, based on tentative reasoning. For each new energy region that becomes accessible, searches for "unknown" phenomena will be made. Some of the most fundamental questions in physics, which go beyond the question of completeness of the entries in the above tables, remain unanswered. For example, searches for magnetic monopoles and free quarks will be continued with higher energies. We do not know why the positive and negative objects in the tables appear in pairs -- this might possibly break down at higher energies. We also do not know today whether leptons and quarks will remain "pointlike" at distances smaller than $10^{-16} \mathrm{~cm}$. Nor do we know whether quarks and leptons are bound states themselves. These questions lead to a need for experiments involving larger transfers of momentum, in turn requiring higher energy collisions than those accessible today and in the eighties.

Free quarks have not been observed, though the indirect evidence for their presence inside hadrons is clear. The mechanism of the confinement is a very active subject of research. One possible mechanism suggests that these may be ne states which correspond to voids of "bubbles" in the vacuum. It is possible that electron or proton collisions are not the best way to create such states, but that collisions between very high energy nuclei would be more effective in forming these states or other unusual states containing the presently known quarks. 
Any such forecast must be approached with some humility. Historically, theoretical predictions for future phenomena in physics have been fallible; in pointing out the success of some theoretical predictions one should not lose sight of past failures. In fact, experience has shown that rarely have accelerators and colliding beam devices been built for the right reason! Although the specific expectations put forward by the proponents of new devices have generally been fulfilled, the most important impact of accelerating and colliding beam devices has generally been in areas not originally foreseen by the proponents.

\section{Economic Consequences and Conclusions}

Over the fifty year history of the field, unit costs measured in terms of \$ per equivalent beam energy have decreased by approximately a factor of a million. This impressive record has been achieved by a series of past devel opments of new techniques. Sti1l, modern acceierators have become more expensive. In an era of constant purchasing power available to the field, future advances in energy will have to be achieved with further reductions in unit cost, such that overall accelerator costs do not increase substantially. This clearly represents an enormous challenge to the accelerator community, and will require considerable R\&D over the coming years on a variety of techniques. Figure 3 shows a projection of the "Livingston Chart" beyond 1980 for both proton and electron machines. 


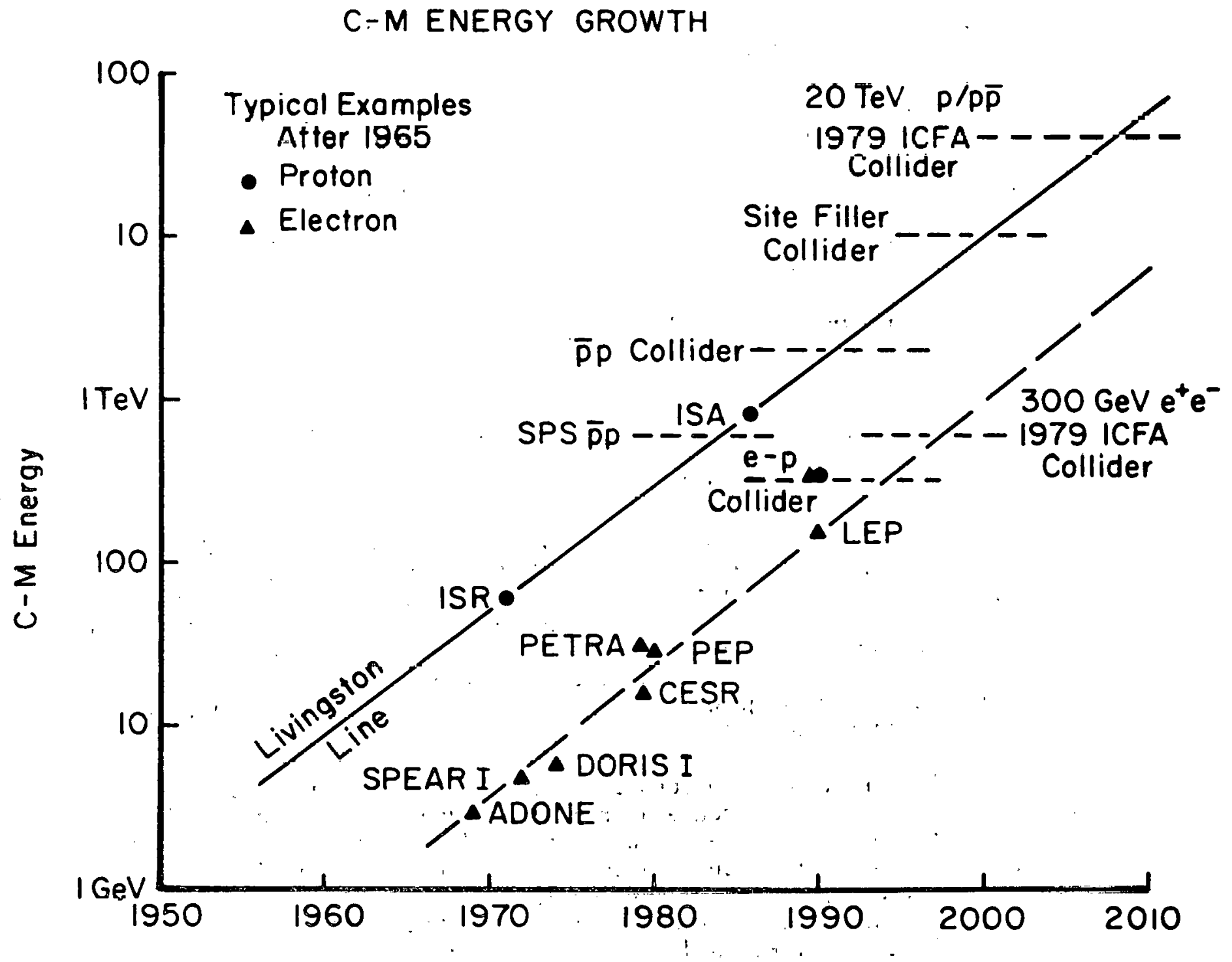

Fig. 3 "Projection of the Livingston Chart Beyond 1980 for both Proton and Electron Colliders" 
II. CHARGE TO THE HEPAP SUBPANEL

ON ACCELERATOR R\&D AND SCOPE OF REPORT

\section{A. Charge}

The HEPAP Subpanel on Accelerator R\&D is to review the overall scope and quality of the accelerator R\&D effort in the U.S. High Energy Physics program. This is to include:

1. An examination of the existing accelerator and detector R\&D effort in the U.S.

2. A comparison of the U.S. effort with efforts abroad.

3. Specific recommendations concerning the U.S. accelerator R\&D effort, with particular emphasis on:

(a) breadth and depth of the R\&D effort;

(b) balance among short-term, mid-term and long-term R\&D;

(c) priorities with in the U.S. accelerator R\&D effort,

(d) appropriate funding levels.

This is to be a broad review. In this sense a central question is whether the planned accelerator R\&D level of effort and content will provide an adequate scientific and technological base for improvement of present accelerators and design of future accelerators that may be needed by the HEP research community. It is assumed that some multiplicity of technological options must be kept open. What accelerator R\&D effort is required to do this? A general approach is needed, and not a specific cuncerll abuul pulenlial construction projects.

The Subpanel is requested to consider detector R\&D and potential new detectors in addition to accelerator R\&D, because of the strong influence that advances in detector technology can have on requisite accelerator and storage ring performance. In this respect the Subpanel is being asked to put their projections for accelerator R\&D within the context of a forecast of future detector capabilities and their implications for long-range accelerator R\&D and not to conduct a broad review of detector lechnology.

An initial report will be made to the Chairman of HEPAP prior to the initial formulation of the FY 1982 budget which will beg in about March 1980. A final report should follow soon after. 


\section{B. Comments on the Charge}

After convening, the Subpanel discussed its charge. It was felt necessary, given the short time available, to limit the scope of the review. It was unanimously felt that it was most important to focus on questions connected with the long-term future of the field. Accordingly, it was agreed to devote the bulk of the Subpanel's detailed considerations and recommendations to the long-range component of accelerator R\&D. R\&D in support of current accelerator projects and detector R\&D were covered only briefly. This decision was conveyed to HEPAP and DUE.

c. Ihe Relationship of Accelerator R\&D to Detector R\&D

Progress in high energy physics research clearly requires ongoing research and development in the accelerator, detector and data processing areas'. While it is true that these fields are interdependent and that developments in one affect developments in another, the pace of accelerator R\&D can be usefully considered independently of questions concerning detector and data processing R\&D.

The fundamental reason for this point of view is the fact that the leading edge of our field is accelerator 1 imited for the foreseeable future. This is not to say that detector R\&D is not needed..- indeed it is if we are to exploit fully our present and future accelerators. Nor is it to deny that there are some fundamental aspects of high energy research that can only be addressed with new and improved detectors. Nor do we disagree that modern digital data processing techniques are crucial to the entire field of high energy physics. However, it is our opinion that any foreseeable accelerator devel opment would be capable of significant utilization with the detector art we have, or can reasonably expect to have. On the other hand, without new accelerator developments, significant regimes of high energy physics simply cannot be reached. The energy parameter is the most obvious example. As we have discussed, beam intensities or luminosities are another.

In a sense, accelerator development comes first, and the accelerator parameters define the set of detector problems that must be solved. Needless to say, the accelerator designer must give reasonable attention to the state of the detector and data processing art in limiting his design parameters. This has certainly been the past history of the field, and we believe it will be the pattern of the future as well. 


\section{PROCEDURE FOLLOWED BY THE SUBPANEL}

In carrying out our charge, we have attempted to gather relevant information and opinion from the widest possible sources. The primary focus of our activities has been a series of six two-day meetings. The first meeting, held in September 1979, was largely organizational in purpose. Subsequent meetings were held in November 1979 and in January, February, March and April. of 1980. During the November, January and February meetings we heard presentations of the HEP accelerator R\&D work at ANL and FNAL, at $L B L$ and SLAC, and at BNL and Cornell. These three meetings were held at FNAL, SLAC and $B N L$ respectively.

All information and opinion-gathering meetings were advertised in advance in the Federal Register. Requests for submission of relevant opinions were published in "Physics Today," February 1980; in the CERN Courier, November 1979; and in a November 1979 mailing to all members of the Division of Particles and Fields of the American Physical Society. Laboratory managements from ANL, BNL; Corne11, LBL, FNAL, and SLAC were invited to address the Subpanel with regard to issues in accelerator R\&D.

At our information gathering meetings we heard, in addition to the laboratory presentations, summaries of work abroad and tutorial summaries of specific areas of'R\&D such as collective accelerators and R\&D in superconducting RF devices. Substantial time at each of the meetings was devoted to discussion of general issues in R\&D and to the organization and classification of the information we had received.

\section{Acknowledgements}

The Subpanel acknowledges with great thanks the very high level of cooperation we have received from all of the laboratories visited and specifically to the large number of individuals at ANL, BNL, Cornell, FNAL, LBL and SLAC who made detailed presentations to the Subpanel. The names and affiliations of these individuals are listed below.

We also recognize with great thanks the hospitality of BNL, FNAL and SLAC in making physical facilities available for our work and to the directors of all of the laboratories participating in our survey for supporting our efforts.

Thanks are also due to the many individuals in Government and University Laboratories and in private industry, both in the U.S. and abroad, who have responded generously to requests for opinion and information relevant to our study. 


\section{Presenters}

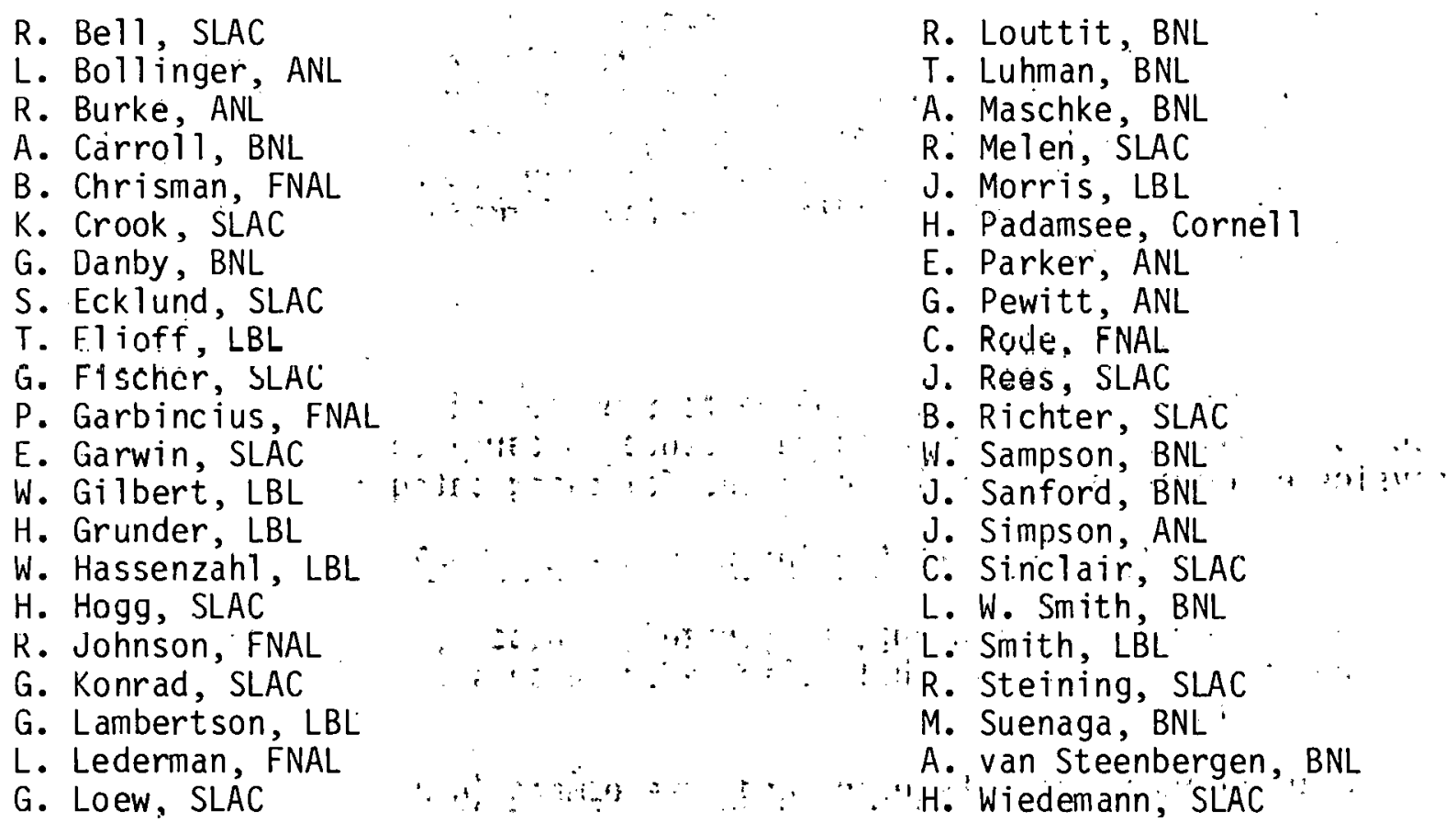

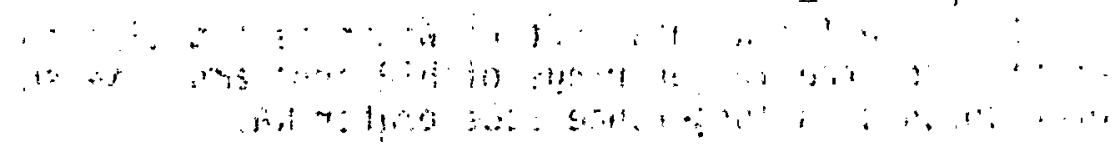

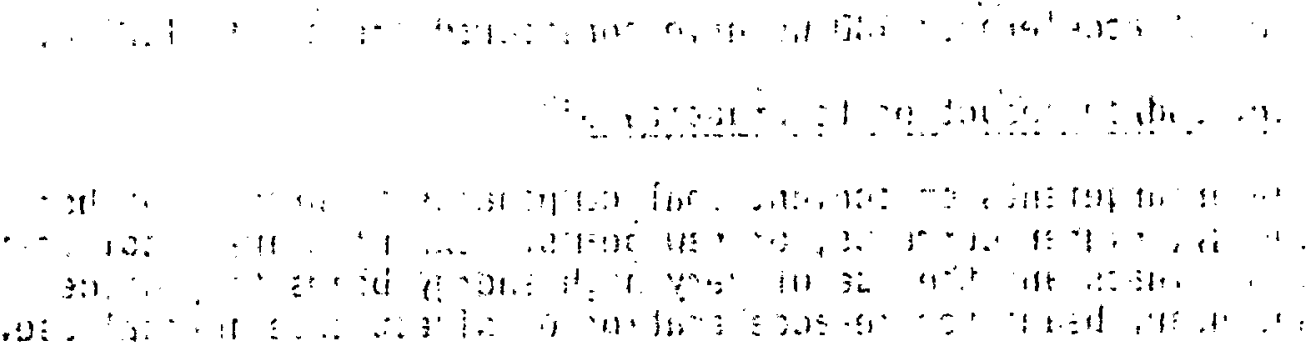

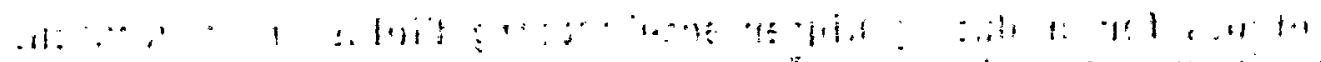

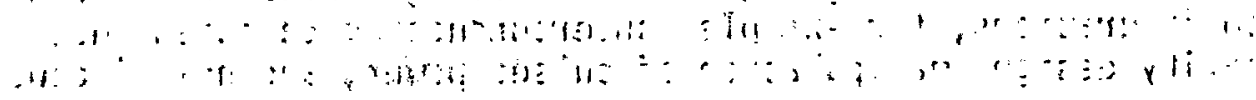

$$
\begin{aligned}
& \text {; : i : }
\end{aligned}
$$

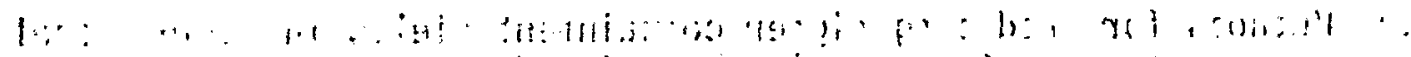

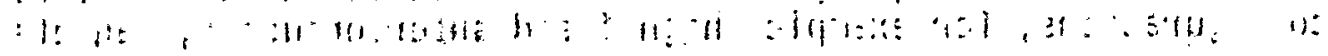

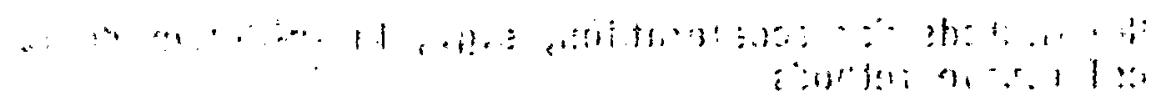


IV. STATUS OF HEP ACCELERATOR R\&D WORK IN THE U.S.

As a central part of our task, we have assembled an illustrative but not exhaustive catalog of accelerator R\&D activities which are directly relevant to the HEP program. The primary purpose of this catalog is to indicate the scope of current work, the magnitude of the overall effort, and the distribution of the effort in terms of short-, mid- and long-range goals. To limit the inevitable ambiguities of definition, we have formulated a working definition of accelerator R\&D.

\section{A. What is Accelerator R\&D?}

The mission of HEP accielerator R\&D is to provide the scientific aind technological basis for the machines needed to carry out high energy physics experiments. We will adopt the following categories:

1. Research and Development Applied to Projects (RDAP)

R\&D addressed to operating, authorized, proposed, or soon to be proposed projects. We shall also refer to this as short and mid-term R\&D.

2. Advanced Accelerator Research and Development (AARD)

R\&D of a more fundamental nature which is anticipated to have benefits for the longer range of HEP programs. We shall also refer to this as long-range accelerator R\&D.

Some examples of accelerator R\&D we have considered are the following:

1. Discovery and/or reduction to practice of:

(a) New arrangements of conventional components to produce higher energy, higher currents, or new beams. Examples are: colliding beam linacs and the use of very high energy beams to produce secondary beams for re-acceleration or direct experimental use.

(b) Methods for producing higher accelerating fields in conventional configurations, for example, superconducting cavities, new cavity design, manipulation of pulsed power, and new RF power sources.

(c) Methods for producing higher containment fields in conventional configurations, for example, high field superconducting magnets.

(d) New methods for acceleration, e.g., laser-driven devices, collective methods. 
(e) New particle sources at low energies, e.g., guns or ion sources for polarized particles, high curręnt fast modulated guns, high power targets for producing $\bar{p}$ or $\mathrm{e}^{+}$.

(f) Diagnostic instruments, e.g., polarimeters, enittance measurement devices.

(g) Methods of improving beam brightness, e.g., stochastic and electron cooling and $\mathrm{H}^{-}$injection.

(h) Beam manipulation devices, such as improved pulsed elements, inflectors, electrostatic or magnetic beam-transport elements, etc.

2. Theoretical, experimental and computer model studies of accelerator ". ".it phenomena such as high current collective effects, non-linear effects, polarization, elc.

3. Studies whose goal is to optimize design through trade-offs within the boundary conditions pertaining to complex accelerator systems.

4. Development of control systems.

5. Development in allied fields such as materials and vacuum science.

B. Current Activities in U.S. Accelerator R\&D

Our listing and description of ongoing work in the field is divided into the thirteen categories shown below. The list is representative and not all-inclusive. This report is not intended as an assessment of the R\&D program of any individual laboratory or person in this country. We have, therefore, generally avoided associating the names of particular laboratories or persons with specific endeavors.

1. Experimental accelerator physics

2. Accelerator theory

3. Systems studies of complete accelerators

4. Superconducting magnets and cryogenics

5. Superconducting RF cavities

6. Normal conducting linac structures and cavities

7. High power RF and microwave sources

8. Stochastic (electronic) and electron cooling of beams

9. Particle sources

10. Beam transport

11. Polarized beams

12. Control systems

13. Non-traditional accelerators 


\section{Experimental Accelerator Physics}

Much of the advancement in accelerator science and technology has and will come from studies which use our present complement of accelerators. Most frequently, experiments with these machines are done with the intention of immediate improvement of their capabilities. However, these studies can often produce results which are not only of special interest but can also be widely applied. Recent examples of such work are the detailed exploration of the non-linear resonances produced by focusing structures; the study of the resistive-wall instability and beam-cavity instabilities in 1 inacs and circular accelerators; the characterization of coupling between transverse and longitudinal degrees of freedom, including the head-tail instabilities and the synchrobetatron resonances; and the attempt to measure the various aspects of the beam-beam interaction in colliding beam machines.

Operating accelerators are used for the testing of new devices and accelerator concepts. Examples are the insertion of superconducting magnets and superconducting RF accelerator devices into external beam Tines or the accelerators themselves for the study of radiation or other environmental sensitivities and of the possible effects on accelerator beam dynamics. Beam-cooling is also being examined experimentally. The effects of very high frequency fields generated by short, intense bunches are actively under investigation in existing machines.

To assess the general level of activity in the use of existing HEP machines for accelerator physics studies, we sent a questionnaire to accelerator laboratories active in HEP requesting information about the topics studied and about the level of resources devoted to this activity. The responses to these questionnaires are summarized in Appendix IV. Typically, 10\% of accelerator running time is used for machine studies. The majority of this time is spent on R\&D to improve the present machine performance, and only one quarter on average is devoted to R\&D for mid- and long-range improvements. The amount of R\&D varies considerably from machine to machine and, as might be expected, tends to be larger for the newer machines.

\section{Accelerator Theory}

The work being carried out in the U.S. HEP 1 aboratories in accelerator theory can be separated into two categories: accelerator "design" and basic theoretical "research". Accelerator design includes magnetic lattice calcultions, injection and extraction system design, effects of magnet non-linearities, specification of alignment toler= ances, RF system studies, and calculations of space charge and other collective limitations. Design also includes systems studies, such as estimates and comparison of colliding linacs versus storage rings and bunched beams versus DC beams in proton storage rinys. The distinguishing characteristic of this work is parameter optimization. 
The second category has the aim of extending our knowledge of accelerators and the physics of particle beams. Examples are: the theory of microwave instabilities, the theory of long-term stability in non-linear fields, the beam-beam interaction in colliding beam systems, and the study of coherent beam oscillations that are not yet completely understood. This category includes new methods of accel eration, for example, colflective field accelerators and laser application to particle acceleration.

In terms of manpower effort, it is important to keep in mind that. those workiny in accelerator theory tend to divide their time between the "design" and "research" catcgorics. We, tlięrture, w111 llse the unils rull-lime equivalent physicists (FTF.).

From information obtained directly from the six large U.S. HEP laboratories, we estimate the total number of physicists working in accelerator theory as 32 FTE. Of these, 11 are in the "research" category, and 21 are in the "design" area. Since the U.S. is supporting a wide range of new projects (the Cornell storage ring which recently turned on, PEP to be operational this spring, ISABELLE under construction, and the Fermilab $\bar{p}$ collider to start construction in the fall of 1980), it is not surprising that almost two-thirds of the effort in accelerator theory is devoted to "design" activity. The fact that short-term goals dominate the theoretical work in accelerators is further confirmed by the paucity of published research in accelerator physics in conference proceedings and refereed journals. This point is illustrated by the following table of the number of papers on HEP accelerator thenry "research" whose origins were in the U.S. laboratories in the years indicated.

$\begin{array}{ll}\text { U.S. National Accelerator Conference } & (1977)-4 \\ \text { U.S. National Accelerator Conference } & (1979)-5 \\ \text { Nuclear Instruments and Methods } & (1979)-0 \\ \text { Particle Accelerators } & (1979)-1 \\ \text { Physical Keview Letters } & (1979)-2\end{array}$

3. Systems Studies of Complete Accelerators

Work in this area ranges from prel iminary design of accelerators to long-term work aimed at defining prohlems relevant to rulure accelerator needs. The major emphasis is on the former category. Significant efforts are in progress on a study of a linear electronpositron collider in the $100 \mathrm{GeV} \mathrm{CM} \mathrm{range.} \mathrm{Other} \mathrm{U.S.} \mathrm{system} \mathrm{studies}$ include an $\mathrm{e}^{+} \mathrm{e}^{-}$storage $r i n g$ for the $100 \mathrm{GeV}$ CM region and an electron ring to be used in conjunction with one (ur more) of the existing or future proton machines to study e-p collisions.

Little long-range systems analysis is currently being done. 
4. Superconducting Magnets and Cryogenics

By far the largest share of the R\&D effort in this field is currently being devoted to supporting the construction of 4 to 5 Tesla magnets for the two major accelerator construction projects at FNAL and BNL. At present, the work focuses on the improvement of magnet fabrication techniques, qual ity control methods for finished magnets and raw materials, and quench-protection. Systems tests and studies are to provide the knowledge necessary for efficient and safe operation of the complete accelerators. Also, tests and calculations are aimed at elucidating and controlling the techanisms responsible for degraded magnet performance, e.g., "training".

There is, in addition, a small amount of important work aimed at the development of future accelerator magnets with fields up to $10 \mathrm{~T}$. This work has several goals: (1) a detailed: understanding of the physics and engineering of superconducting magnet behavior, taking account of the larger forces and stored energy densities in the higher field magnets; (2) evaluation and development of $\mathrm{NbTi}, \mathrm{Nb}_{3} \mathrm{Sn}$, and ternary conductors and fabrication techniques for the conductors; (3) development of prototype magnets using geometries other than the Cosine-theta design; and (4) systems studies taking cooling, materials, and metallurgical problems into account.

Accompanying this work on magnets has been a considerable amount of project-oriented development of cryogenic apparatus in general and refrigeration systems in particular. The refrigerators to be used for the accelerator projects now underway will be the largest liquid helium sysleils ever built and operated. The primary goals in current development efforts are the assurance of operating reliability and the maximization of efficiency.

Attention is being devoted to development of turboexpanders and the removal of oil from the warm helium stream when wet screw compressors are used.

Systems for refrigeration below the lambda point are also in need of development for high field superconducting magnets and RF cavities.

5. Superconducting RF Cavities

RF superconductivity is a technology that holds promise for high dccelerating ficlds with low power input. Excessive power losses and multipactoring at $h$ igh accelerating fields proved discouraging to the many workers who tried to develop the technology five to ten years ago. The continuing need for economical RF acceleration with high fields, some modest advances in the understanding of previously observed limitations, and improvements in surface science instrumentation have sparked renewed interest in the field. Activities now in 
progress in the U.S. HEP laboratories include surface studies aimed at the suppression of secondary emission and at understanding mechanisms of excessive local surface losses. Other studies focus on cavity quenching. The principal material being investigated for HEP accelerator uses is niobium. Refrigeration efficiency could be improved if a superconducting material with a higher transition temperature could be found. $\mathrm{Nb}_{3} \mathrm{Sn}$ and other materials have been tried in the past but found intractable. At present a small effort to make $\mathrm{Nb}_{3} \mathrm{Sn}$ cavity surfaces by a solid-state-diffusion method is underway.

In addition to fundamental studies. cavity design is proceeding with the aim of devising shapes that suppress multi-pactoring, that are simple tu rabricate, and that al so allow almost complete extraction of power coupled from the beam in unwanted modes.

Considerable effort is also being put into the use of RF superconductivity for intermediate energy (below $1 \mathrm{GeV}$ ) accelerators which will aid in the fundamental understanding for HEP applications.

6. Normal Conducting Linac Structures and Cavities

Work on this subject may be divided conceptually into three categories: (1) studies of existing structures to determine their limiting properties; (2) attempts to improve performance of existing designs through relatively minor changes; and (3) development of completely new designs having considerably enhanced properties. In category (1), there is an active program to measure the beam-cavity interaction for very short high-intensity bunches, a subject of great importance for electron colliders. In category (2), there are commercial efforts to increase substantially the gradients of more or less standard structures. In categnry (3), activity is focused on development of the so-called disk and washer structure within the U.S. nuclear physics program. There is also a small effort to examine structures suitable for use with very powerful sources of optical, millimeter, or submillimeter waves. Finally, there is work on RF quadrupole linac structures which provide both focusing and acceleration at low particle velocities.

7. High Power RF and Microwave Sources

Currently only one of the HEP laboratories is engaged in R\&D on RF sources. While the bulk of the effort is in improving the performance of existing tubes, a small amount of work is devoted to development of a klystron in the $100 \mathrm{MW}$ range and of a high efficiency tube of the rotating beam type. The U.S. Nuclear Physics program is supporting a small amount of work on the high-efficiency "gyrocon" concept for application in the UHF band. 
Considerable tube development is being carried out by commercial firms under defense contracts or by the Naval Research Laboratory alone or in cooperation with various university groups. After some further developments, sone of these devices may have direct application in HEP accelerators. The following is a list of some of the devices which operate in frequency bands now used in accelerators:

\begin{tabular}{lcc} 
Tube Type & Band & Peak Power \\
\cline { 2 - 3 } & $\mathrm{S}$ & $500 \mathrm{MW}$ \\
Klystron & $\mathrm{S}$ & $1 \mathrm{GW}$ (built but not tested) \\
Cyclotron Maser & $\mathrm{X}$ & $0.9 \mathrm{GW}$
\end{tabular}

These tubes have beam voltages of.several MV and currents in the kiloampere range.

Another interesting approach is to charge a very high-Q cavity from a CW or long pulse source of moderate power and then to dump the energy from the cavity rapidly by use of a fast-acting switch. The switch in combination with a suitable microwave coupling circuit can effect a large and rapid change in the coupljing between the cavity and the external load. Power gains of $5 \times 10^{4}$ have been realized in the kilowatt peak-power range.

8. Stochastic and Electron Cooling of Beams

Part of the ongoing R\&D program is aimed at a fundamental understanding of stochaslic cuuling, and how to improve its utility. Therefore, it includes the theory of stochastic processes, low-noise electronic systems, and advanced beam detectors. Two systems have been built, one to damp transverse oscillations and the other to damp momentum spread. Experiments have begun in a small ring, and momentum cooling has been demonstrated. Another part. of the program is aimed at a proposed construction project for which emphasis is placed on momentum cooling. Activities in the program include theory and simulations, as well as pick-up, kicker and electronics development.

Electron cooling R\&D has been directed at the development of systems of such a scale as to be useful for real istic accumulation schemes. New diagnostics arc being developed to measure the electron beam properties. An advanced electron-collector system allows recovery of $99.9 \%$ of thc electrons from the rathode and energy recovery of $95 \%$. The conditions necessary to obtain stable operation of the electron system are being studied. This program has entailed interlaboratory collaboration in the design of the gun and collector and in the correction system for the storage ring. The system has been largely completed and is under operational tests. The goals of the experiment are to achieve accumulation of protons by multiple injection and to study high current effects at intensities interesting for a prolonantiproton colliding beam system. There has also been a small 
activity to extend these studies to the electron cooling of high energy beams.

\section{Particle Sources}

There is considerable work on ion sources for $\mathrm{H}^{-}, \mathrm{H}^{+}$, heavier ions and polarized particles within the HEP program and in the nuclear physics and fusion programs. While there are many aspects of sources that require continuing investigation, it is felt that the current level of activity will suffice to meet foreseeable needs.

Recently substantial progess has been registered in electron sources through the devclopment of the lasel eleclrun gun. In this device the beam (highly pularized) is generated by photoemission from a cesiated GaAs solid state cathode bombarded by polarized laser light. Peak currents of severa:l'tens of amperes have been achieved in short bunches.

\section{Beam Transport}

Several interesting and important ideas in the transport of bright, high energy electron beams as well as bright, high intensity but low energy electron and ion beams are under intense investigation. The high energy electron work is mainly in conjunction with the linear collider mentioned previously. The problems is to bring a very high energy beam with signficiant energy spread to a very sharp (2 micron) focus without the use of massive magnets close to the focal point. The final focus will be created by means of permanent magnet lenses using a new material (samarium-cobalt) which has the very useful property that its incremental permeability is unity. Broad-band local chromatic corrections are needed and are under design. In addition, one has to transport the high energy beam over a curved path while minimizing the effect of quantum fluctuations in the synchrotron radiation on the phase space density of the bearil.

For the focusing of intense low energy beams, an interesting system involving large parallel arrays of miniature electrostatic quadrupoles is under development. It is estimated that very bright, high current beams can be transported and accelerated with minimum space charge effects. For HEP applications such devices could aid in the design of compact injectors and might facilitate the construction of very high power RF and microwave tubes.

11. Polarized Beams

In recent years important advanced have been made in the production of polarized proton beams. Currently, there are two collaborative efforts to apply this work to still higher energy beams. One approach is based on the acceleration of highly polarized protons from a low energy source to high energy in an $A G$ synchrotron. The depolarizing 
resonances encountered during acceleration are avoided by the use of pulsed quadrupoles. The other approach will provide polarized protons or antiprotons from a secondary beam derived from the decay of polarized lambda hyperons. This beam line will have special magnets to control the direction of the proton or antiproton spins on a pulse to pulse basis.

For electron machines, a number of polarized sources have been developed, one being the photoemission gun mentioned above. Development of this source continues with the goal of improving intensity and polarization. Polarizations in excess of $80 \%$ may be achieved.

Studies of the stability of spin motion in electron storage rings with emphasis on providing longitudinally polarized beams are an important aspect of design studies for future possible electronpositron colliding beam devices.

\section{Control Systems}

The rapid and continuing progress in microelectronics has revolutionized accelerator design. Sophisticated systems for the coordinated manipulation of accelerator parameters, which ten years ago would have been impossible, are now common place. The influence of this revolution is so pervasive that even a partially inclusive catalog is beyond the scope of this report. Suffice it to say that the accelerator community generally has been very adept at capitalizing on each new electronics development. The achievement of reduced operating costs and increased "up time" by sophisticated electronic diagnostics, alarms, and controls have allowed the reduction of operating staffs.

13. Non-Traditional Accelerators

(a) Background

All operating HEP accelerators use magnets and/or accelerator cavities which are direct descendents of the machines of the $30^{\prime} \mathrm{s}$ and $40^{\prime} \mathrm{s}$. The confinement fields are produced by assemblages of solid conductor and ferromagnetic material. Recent years have seen some serious attempts to break out of this "old" pattern and by so doing to circumvent the limitations of traditional materials and methods. The bolder approaches have focused on the production of very high confinement and accelerdtion fields directly by manipulation of high-power chargedparticle beams in vacuo, or in a plasma medium, or by the manipulation of the charges in a plasma through the action of very intense, very short wavelength radiation passing through the plasma. Other approaches focus on the possible large enhancement of linear accelerator gradients by the use of very high power, very high frequency radiation as from a 
laser. Yet other activities center on the acceleration and/or confinement of beams having currents much larger than that normally utilized in HEP accelerators. At present, none of these have come to practical fruition in new HEP accelerator machines. Nevertheless, the pursuit of these ideas, and others with similar goals, are an essential part of HEP accelerator R\&D.

A substantial portion of this work, in monetary support terms, is conducted outside the HEP program. As a consequence, knowledge of its details is not widely spread in the H[P communily. Fur informaliullal purposes, more details are given in Appendices I, II and III which describe representative ongoing work.

(b) Non-collective-effect "áćcëlerators for high-currents

At this time, there is experimental work underway on a number of devices. Among them are an RF linac for high brightness ion beam acceleration, a high-current proton storage ring, several induction linacs for kiloamp beams, and the auto-accelerator in which energy is transferred between a high current charging beam and a relatively small acclerated beam.

Another interesting device, studied only through calculation so far, is the extension of the microwave linear accelerator to optical frequencies. In such a device, the klystron source is to be replaced by a laser and the cavity structure by an optical equivalent such as an assemblage of gratings. It is estimated that effective gradients in excess of $1 \mathrm{GeV} / \mathrm{m}$ might be realized with such a device if the very significant practical problems could he overcome.

\section{(c) Collective-effect accelerators}

These accelerators can be divided into two broad categories. In one category are accelerators in which the primary purpose of the collective effects is to produce transverse confinement. In the other category, the collective effects produce longitudinal acceleration as well as confinement. Among the former are the magnetically insulated electron-focused ion linac, in which trapped clouds of electrons form a transversely focusing potential well for the ions; and the toroidal electron-focused ion accelerator, which works in a similar way but has a circular equilibrium orbit.

Among the latter are several different types of linear accelerators:

1. The ion-containing electron-cluster accelerator in which an electron bunch containing relatively few ions is 
accelerated in some type of linac. In these arrangements, the energy gain of the ions will be roughly (ion mass)/ (electron mass) $x$ electron energy gain. Past work on these devices, such as the Electron Ring Accelerators, revealed great difficulties in their realization, and very little activity continues in the U.S.

2. Moving-potential-well accelerators exist in several manifestations, most of which involve high current electron beams being shot through foils into plasma volumes with various auxiliary focusing devices. These accelerators are characterized by rather low ion energies with large currents and appear to have little interest for HEP applications directly. They are described more fully in Appendix I.

3. Two devices are being developed which use travel ing waves "grown" on an intense relativistic electron beam to achieve acceleration and focusing. In one, cyclotron waves with tapered phase velocity are grown on the beam. In the other, the more familiar space-charge waves are grown on the beam as it passes through an exciting slow-wave structure. Conceivably, such accelerators might find some direct use in HEP machines or be part of very high-power RF, microwave or optical amplifiers whose radiation output is used to drive an accelerator.

4. Another interesting class of accelerators has been proposed in which the very high fields at the focus of a laser beam (or other beam of millimeter or sub-millimeter wavelength) gather up a high-density pulse of electrons from a low energy electron stream or dilute plasma and propel it forward with a controllable group velocity. Ions can then be acceleratd in the wake of this moving charge pulse. It is thought that gradients of $30 \mathrm{GeV} / \mathrm{m}$ might be achieved. So far only analytical estimates and some computer simulations have been carried out. In Appendix II, several of the suggested "laser" accelerators are reviewed briefly and a representative bibliography is given.

\section{(d) Power Sources}

The practicality of several of the very high gradient linear acceleration devices mentioned above depends in part on the existence of very high peak power sources in the millimeter to optical bands. A wide range of work is being carried out within the fusion and defense programs, and is reviewed briefly in Appendix III. 
C. Resources Devoted to R\&D

The resources devoted to work that is clearly of a long-range nature are quite small, certainly not more than $\$ 3 M$ per year.

By far the greatest portion of monetary and manpower resources in R\&D is applied to the support of operations and approved construction projects, with a much smaller amount applied to anticipated construction proposals. We would classify this as short- to mid-term R\&D (RDAP). Figure 4 is a graph of R\&D support as a fraction of the total HEP operating budget, which is abuul \$200M in this riscal year (FY UO). Currently, the total expenditure for accelerator R\&D is about $14 \%$ of operating funds, $3 / 5$ of which is devoted to superconducting magnet R\&D in support of the two current major construction projects.

D. Manpower Resources in Accelerator R\&D

We have attempted to study the utilization and the supply of Ph.D. level professional scientists working in the accelerator field. For the study of the characteristics of the field, we have used the data presented in the 1978 census of the U.S. High Energy Physicists and Graduate Students (DOE/ER-0019, November 1978). For questions relating to the future supply of accelerator scientists, we chose to concentrate on the group which entered the field during the last five years. The data presented were compiled from information supplied by the five U.S. National Laboratories - Argonne, Brookhaven, Lawrence Berkeley, Stanford Linear Accelerator and Femilab - which have had substantial high energy physics related accelerator work in progress during the last five years.

Figure 5 shows the age distribution of accelerator physicists. As can be seen, there is little difference between the age spectra of those who reported accelerator physics as their primary activity or as their secondary activity. The mean age is around 49 years and the distribution is broad. Figure 6 shows the age distribution by subfield. Of some concern is the rather old age distribution of accelerator theorists as of 1978. The figure aiso illustrates that the majority of accelerator physicists (84\%) are engaged in design and development while relatively small segments are primarily involved in theory (9\%) and operations (7\%).

In general, the average age of the accelerator physicist is about 49 years and the distribution has a broad peak at age 40 years with very few in the 25 to 35 year range. We have found that this distribution reflects the fact that many accelerator physicists have entered the field substantially after receiving their Ph.D. in other areas. Figure 7 shows the distribution in age at the time of entry into the accelerator field for entrants during the last five years.

Figure 7 shows a nearly flat distribtion between ages 25 and 45 indicating a pattern of del ayed entry into accelerator physics. In the comments made by the Laboratory managements, in response to our requests for manpower 


\section{Technology R \& D}

Acceleriotor R \& D

Experimental Facility $R \& D$

as $\%$ of total operoting funds

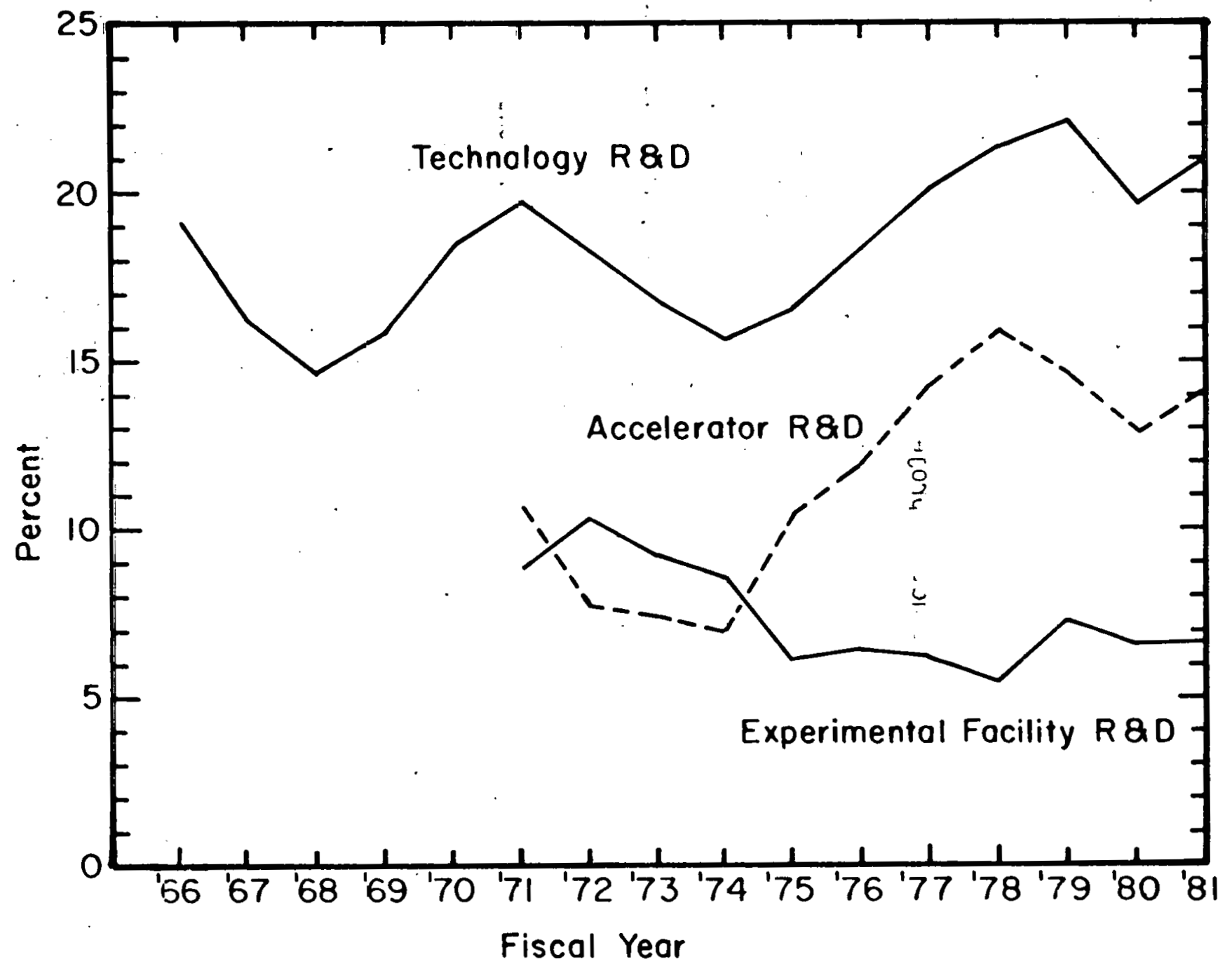




\section{Age Distribution of Accelerator Physicists}

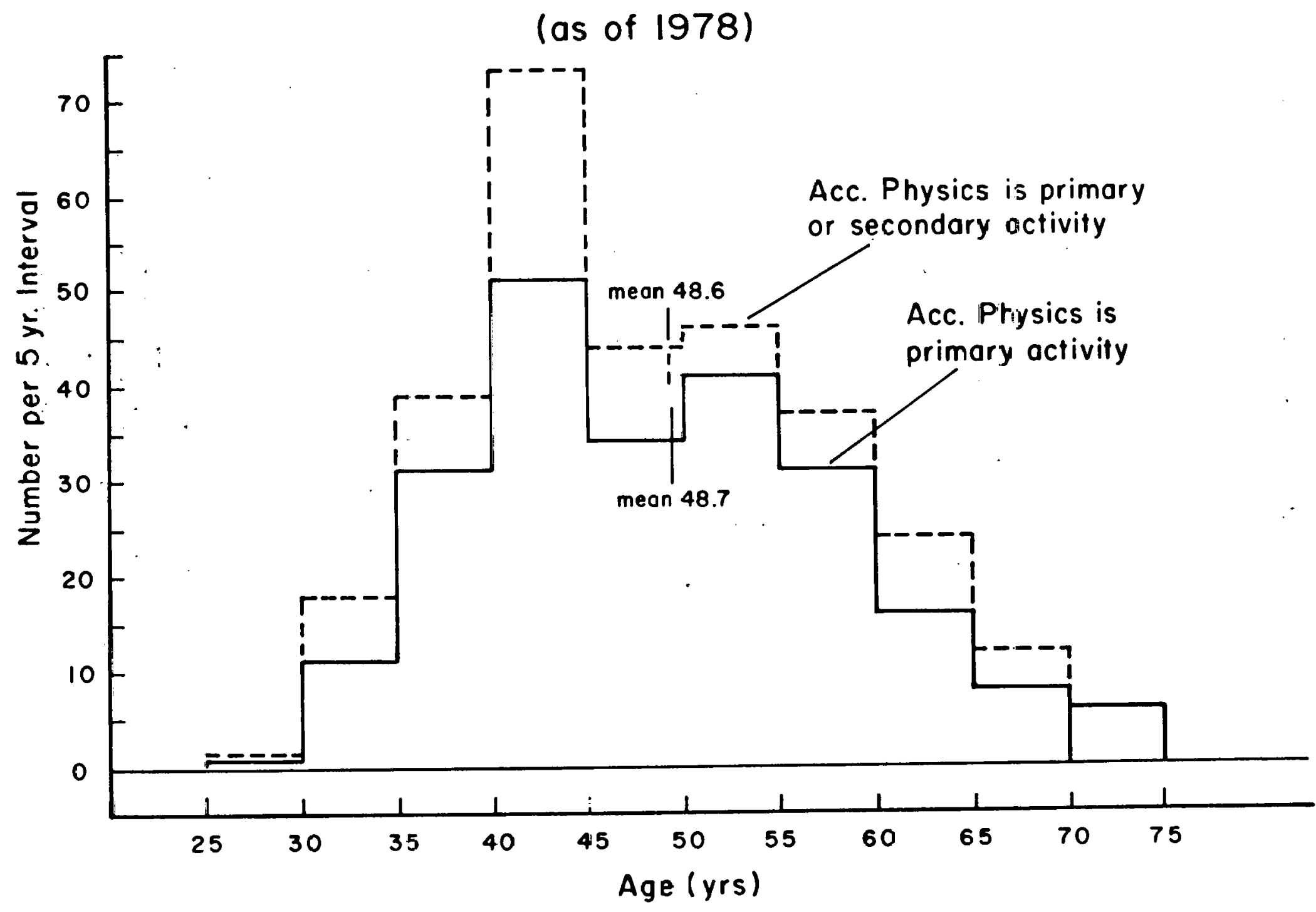

Fi. 5 


\section{Age Distribution of Accelerator Physicists by Subfield (1978 Data)}

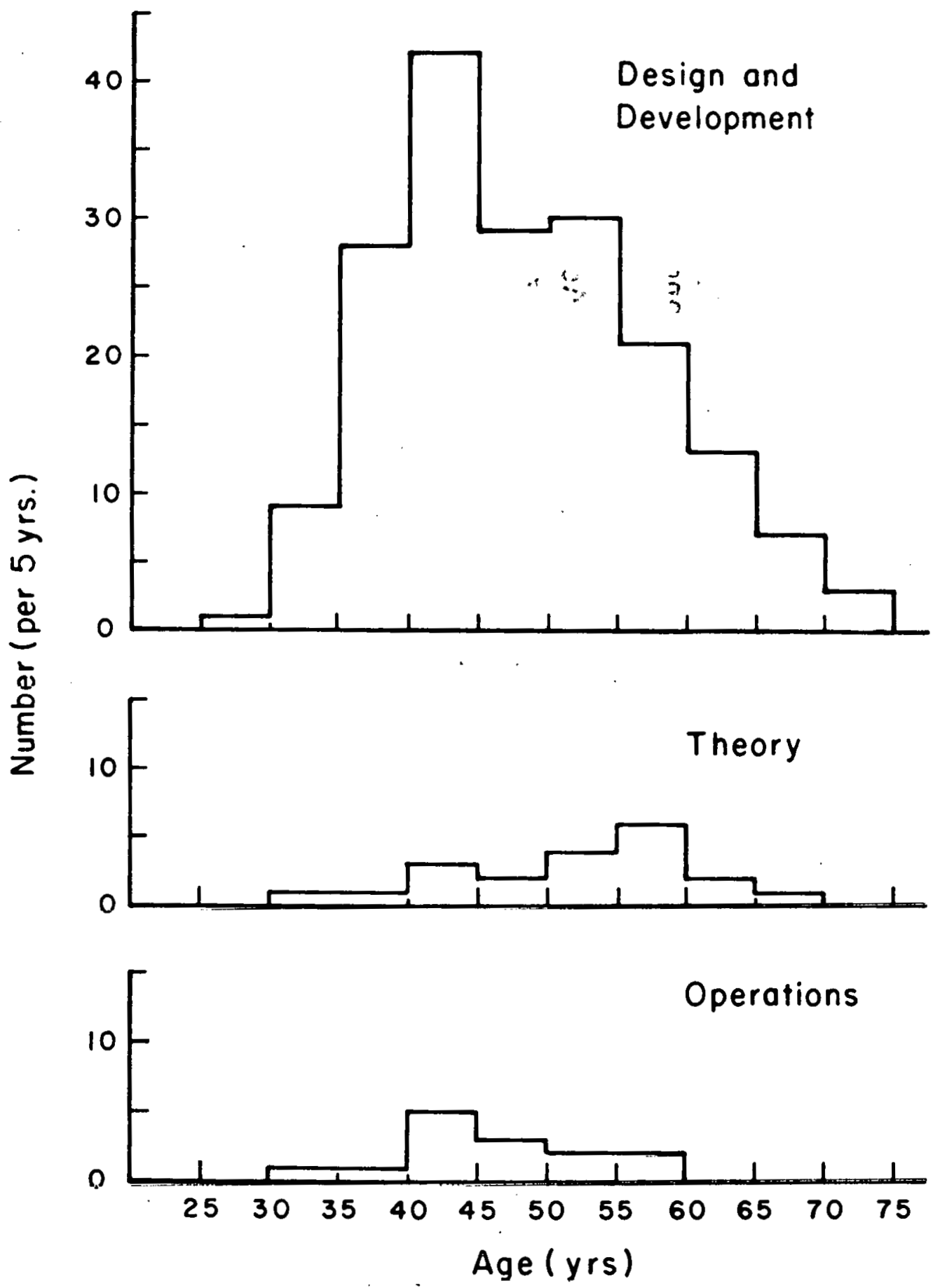

Fig. 6 
Distribution of Age at Time of Entry to Accelerator Physics (Last 5 years)

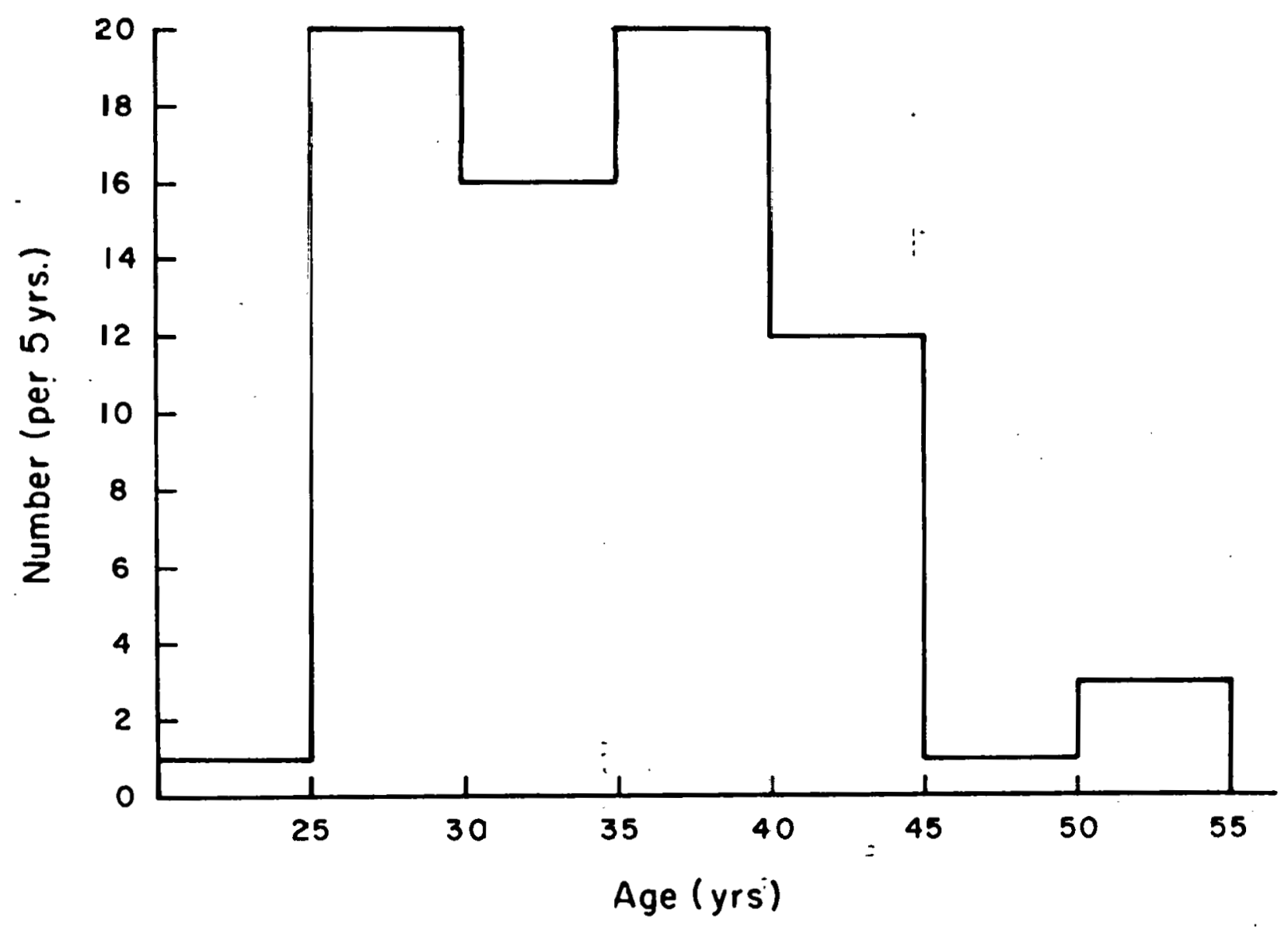

Fig. 7 
information, there was a common theme of stressing the importance to the accelerator field of these post Ph.D. entrants.

We have also determined the field of the Ph.D. dissertation for the entrants of the last five years as shown in Table 4 bel ow.

\section{Table 4}

\section{Distribution by Field of Ph.D. Thes is for Entrance to} Accelerator Field in the Last Five Years

\begin{tabular}{|c|c|c|c|c|c|c|c|}
\hline Ph.D. Thesis Field & ANL & BNL & Cornell & $\underline{L B L}$ & FNAL & $\underline{\text { SLAC }}$ & Total \\
\hline Accelerator Physics & & 1 & 3 & 2 & & 1 & 7 \\
\hline $\begin{array}{l}\text { Experimerital High } \\
\text { Energy Physics }\end{array}$ & 2 & 2 & 2 & 2 & 26 & 3 & 46 \\
\hline $\begin{array}{r}\text { Theoretical High } \\
\text { Energy Physics }\end{array}$ & 1 & 3 & & 1 & 1 & 1 & 7 \\
\hline Plasma Physics & 1 & & 1 & 3 & & & 5 \\
\hline Other & & 4 & & 3 & 2 & 1 & 10 \\
\hline & & & & & \multicolumn{2}{|c|}{ TOTAI. } & 75 \\
\hline
\end{tabular}

Table 4 shows that al though there are diverse sources of accelerator physicists, a very important, even dominant, component is high energy physics. It should be noted that there are a number of physicists who illake cuntributions to both particle and accelerator physics. In fact, it is this overlapping involvement which gives a great deal of strength to high energy physics.

Al though the numbers of working accelerator physicists trained initially in the field is small, it is in a qual itative sense very important. These people tend to be broadly trained and will play a role greater than their numbers indicate in the areas of long-range accelerator research and development. In addition, the existence of faculty and dissertation programs in accelerator physics is very important for the collection and communication of results obtained in the field. Many of the post-Ph.D. entrants to the field gain their knowledge of the subject from key books and articles as well as by their "on-the-job" training in their new profession.

We have also compiled data on the Ph.D. institutions of the entrants of the last five years. These are presented in Table 5. At the multifunction 
laboratories, a fraction of the physicists enumerated in Table 5 work in non-HEP accelerator areas. This fraction is small and its exclusion would not change the overall pattern.

Table 5

Ph.D. Granting Institutions

for Entries into Accelerator Field in Last Five Years

Institution

U. of British Columb1a

U. of Calif.-Berkeley

U. of Calif.-Irvine

U. of Calif.-Los Angeles

Cal. Tech.

Case Inst. of Technology

U. of Chicago

Columbia

Cornell

Harvard

U. of Illinois

Iowa State U.

Johns Hopkins

$U$. of Maryl and

Mass. Inst. of Tech.

U. of Michigan

Minnesota

North Texas U.

U. of Pennsylvania

Pittsburgh

Princeton

Purdue

U. of Rochester

Rutgers

Stevens Inst. of Tech.

Stanford

SUNY, Stonybrook

Tufts

$U$. of Washington

U. of Wisconsin

Yale

Foreign
BNL FNAL LBL SLAC ANL Cornell Total

$\begin{array}{lllllll}- & 1 & - & - & - & - & 1 \\ 1 & 4 & 1 & - & 2 & - & 8 \\ - & - & 1 & - & - & - & 1 \\ - & - & 1 & - & - & - & 1 \\ - & 1 & - & - & - & 1 & 2 \\ - & 1 & - & - & - & - & 1 \\ - & 3 & - & - & - & - & 3 \\ - & 2 & - & 1 & - & 4 & 6 \\ 2 & - & - & - & - & - & 2 \\ - & 1 & 1 & - & - & - & 3 \\ - & 3 & - & - & - & - & 1 \\ - & 1 & - & - & - & - & 1 \\ - & 1 & - & - & - & - & 3 \\ - & - & 3 & - & - & 1 & 6 \\ 2 & - & 1 & - & - & - & 2 \\ 1 & 1 & - & - & - & - & 1 \\ 1 & - & - & - & - & - & 1 \\ - & 1 & - & - & 1 & - & 1 \\ 1 & - & - & - & - & - & 1 \\ 1 & - & - & - & - & - & 2 \\ 1 & - & - & - & 1 & - & 1 \\ - & 1 & - & - & - & - & 1 \\ - & 1 & - & - & - & - & - \\ 1 & - & - & - & - & - & 1 \\ - & - & - & 1 & - & - & 2 \\ - & - & - & 1 & - & - & 1 \\ 1 & - & - & - & - & - & 2 \\ 1 & - & - & - & - & - & 4 \\ 1 & 1 & - & - & - & - & 2 \\ 1 & 3 & - & - & - & - & 9 \\ 2 & - & - & - & - & - & 75 \\ 2 & 3 & 3 & 1 & - & 6 & \\ 19 & 29 & 11 & 6 & 4 & 6 & \end{array}$


In summary, we find that the supply of accelerator scientists while perhaps marginal, is not in a critically low state. A key component in the supply is the migration into the field by physicists trained in other areas, the most important being experimental high energy physics. It is clear to us that this will continue to be the case for some time. Activities such as graduate courses, lecture series at the laboratories, etc., which educate high energy physicists in aspects of accelerator physics and which convey some of the intellectual content and challenge of the field are important to the continued, necessary recruitment of accelerator scientist and should be encouraged. While the number of accelerator physicists originally trained in the field is small, they and the programs which produce them are important and should be encouraged and supported.

\section{E. Resource Allocation for Accelerator R\&D}

A very small amount of R\&D is carried out by university groups operating independently of the national accelerator labs. Proposals are made directly to'the agencies and subjected to the same peer review process used for HEP: particle physics research activities. Some work is carried out by university groups in collaboration with the large accelerator labs. In these cases, the initiative may be from the university or shared with the labs; funding may be through the laboratories or by some

i. arrangement with the agencies.

At the national laboratories, the patterns are diverse. Initiatives come from directors, laboratory accelerator staffs, in-house HEP physicists and sometimes from "user" physicists. These may be reviewed by the laboratory management, alone or with the advice of various program advisory or visiting'committees. In consequence, internal laboratory funds are allocated, or formal proposals may be forwarded to the agencies for review and support.

The flexibility for reallocation of internal laboratory funds is closely related to the total amount of HEP funding at a given laboratory. At the multipurpose laboratories this flexibility may be limited by the ongoing needs of other'programs for accelerator expertise and relative priorities must be set by the laboratory management. On the other hand, the management can draw upon expertise, normally supported by other programs at these laboratories, both in the accelerator field and in other disciplines.

Accelerator R\&D is a part of the "technology" line item in DOE (but not NSF) budgets, but the accounting and reporting practices with regard to it vary among the labs, which makes meaningful comparisons difficult. Nevertheless, it is clear that the larger HEP operating budgets of the HEP accelerator laboratories allow considerable flexibility in the." continual tuning of the balance among various R\&D activities and in the rapid start-up of termination of small R\&D activities.

The above remarks do not apply to R\&D in support of approved construction projects. These funds are more formally programmed and managed as an integral part of the construction projects. 


\section{ACCELERATOR R\&D EFFORTS ABROAD}

Accelerator R\&D outside the U.S. is concentrated in three well-defined international regions: Western Europe, the USSR and Japan. These geographic subdivisions comprise those advanced nations whre the combination of cultural impetus and economic resources naturally lead to the pursuit of an advanced research enterprise such as high energy physics. One also finds in these regions the broad base of advanced technology which forms the essential foundation for the successful pursuit of such research.

\section{A. HEP Accelerator R\&D in Europe}

Accelerator R\&D in Europe is concentrated largely at CERN and DESY, with some accelerator-related R\&D taking place in a few other laboratories.

\section{Accelerator Physics}

Accelerator physics studies are carried out on the PS, ISR and SPS at CERN, and on PETRA and other accelerators at DESY. Approximately $20 \%$ of the running tme of the ISR is devoted to machine development; the corresponding figures at the SPS and the PS are 15\% and 10\%, respectively. For PETRA, which is still in development, the figure is $30 \%$. Much of this work is directed toward improving machine performance, but a significant fraction has longer term aims and implications. Examples are the original development of stochastic cooling on the ISR, studies of "microwave" instabilities on the PS and the SPS, 1 ifetime of bunched beams on the SPS, and the beamabeam interaction on the ISR. The luminosity of PETRA is smaller than planned by about an order or magnitude, and much machine study is devoted to understanding the limiting effects. This has the shortterm aim of improving the luminosity, but the understanding of this problem may have long-term implications for LEP or LEP-like projects. Studies are also devoted to the upgrading of PETRA to $22 \mathrm{GeV}$ per beall.

CERN has built a special beam cooling experiment, ICE, on which experimentation with stochastic and electron cooling was performed.

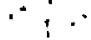

2.: : Ndvanced Technology

\section{(a): Superconducting Magnets}

Studies of superconducting accelerator magnets started long ago at the Rutherford Laboratory, Saclay, and Karlsruhe. In 1970, these laboratories joined with CERN in a collaboration, GESSS, for coordinating their activities toward equipping the SPS with superconducting magnets. Since then, the original purpose has fallen to the background, and these laboratories have turned to other applications of superconductivity, e.g., fusion. 
CERN has continued work on superconducting magnets for beam transport in the experimental areas of the SPS (in collaboration with Saclay) and for a low-beta insertion in the ISR.

of longer term interest is the work in France (Saclay and Grenoble) on high field magnets based on $\mathrm{NbTi}$, and the $\mathrm{Nb}_{3} \mathrm{Sn}$ work at the Rutherford Laboratory.

(b) RF Superconductivity

Karlsruhe has been active in the field of RF superconductivity for many years. They built a small proton linac with a hel ix structure and encountered problems (multipactoring, etc.) that limited the accelerating fields to modest values. In collabora$\therefore \because ; \cdot$, tion with CERN, they built a superconducting IRF separator, which has worked very reliably for several years.

A collaboration involving CERN, DESY and Karl sruhe is making model superconducting cavities to be tested on DORIS. RF. superconductivity work has also gone at Wuppertal (Germany), Genoa (Italy) and Orsay (France), on a smaller scale. Currently; all these laboratories are pooling their efforts with CERN to make experimental cavities aimed specifically at LEP application.

(c) Vacuum Development

$R \& D$ on vacuum technology has high priority at CERN in the. continuing effort to improve the performance of the ISR.

3. Project-oriented R\&D

Currently, there is one accelerator project under construction in Europe and several others are under study. All of these required extensive R\&D of various kinds. We list main fcatures below.

\section{(a) $\mathrm{p} \overline{\mathrm{P}}$ at CERN}

The main aim is to achieve $2 \times 270 \mathrm{GeV} p \bar{p}$ collisions ing two.
experimental areas of the SPS, with a luminosity of $10^{3 \mathrm{~cm}^{-2} \mathrm{~s}}-1$ in each. This project relies on stochastic cooling for accumulating enough antiprotons, and extensive theoretical and experimental ICE studies are near completion. Studies, both theoretical and experimental, have been undertaken on lifetime limitations, the beam-beam interaction, and other instabilities.

The ISR is also being prepared to receive antiprotons. In addition, there is a program for low. energy antiproton beams. . 
(b) LEP

The study of LEP is centered at CERN but is carried out in collaboration with ECFA and most of the HEP national laboratories in Europe. LEP's main parameters are:

$\begin{array}{ll}\text { Energy } & 2 \times(50-130) \mathrm{GeV} \\ \text { Luminosity } & 0.4-1 \times 10^{32} \mathrm{~cm}^{-2} \mathrm{~s}^{-1} \\ \text { Circumference } & 30.6 \mathrm{~km} .\end{array}$

Many pcople work full-time on the study of this prnjert. It. is difficult to distinguish between R\&D and design based on advanced technology, but a considerable fraction of the effort should be classed as R\&D. Examples are beam studies and optimization of parameters to steer clear of instabilities up to design luminosity. The RF superconductivity studies have been mentioned earlier, and the highest energy quoted above assumes superconducting cavities. "Pulsed" conventional cavities are also being studied.

(c) HERA

The study of this project is centered at DESY but assistance is drawn in from ECFA, CERN and other laboratories. It is a colliding-beam facil ity with an electron ring interlaced with a superconducting proton $r i n g$ :

Energy

Luminosity

- longitudinally molarized

$$
30 \mathrm{GeV} \text { e }-820 \mathrm{GeV} \mathrm{p}
$$

$$
10^{31}-10^{32} \operatorname{cill}^{-2} s^{-1}
$$

\section{Circumference \\ $6.5 \mathrm{~km}$}

The project study leans heavily on the experence being gained at PETRA. Superconducting magnet R\&D is being carried out in collaboration with other institutions.

B. Accelerator R\&D in Japan

The accelerator R\&D work in Japan is managed and funded as a small part of the effort of 1 aboraturies operating large accelerator facilities. Because experimental high energy physics is a relatively new endeavor in Japan, the large laboratories are fewer in number and not as large as those in the U.S. Compared to the U.S., they do receive much more assistance and support from industry, where a great deal of capability 
and depth for doing accelerator R\&D work resides. Japanese high energy laboratories operate with a relatively small accelerator R\&D staff and contract most of the R\&D to industry. The only two high energy laboratories in Japan are the National Laboratory for High Energy Physics in Tsukuba and the Institute for Nuclear Study of the University of Tokyo.

1. National Laboratory for High Energy Physics (KEK)

This laboratory built and is now operating a $12 \mathrm{GeV}$ proton synchrotron. The accelerator R\&D program is mostly related to the proposed high energy colliding beam facility TRISTAN (Transposal Ring Intersecting Storage Accelerator in Nippon). This facility would consist of two intersecting rings having an average radius of $432 \mathrm{~m}$. The electron $\therefore$ ring will be used for $2 \times 30 \mathrm{GeV} \mathrm{e}^{-}$colliding beams and will be

$:$ : composed of conventional magnets and superconducting RF cavities.

Four accelerator physicists are engaged in the development of superconducting RF accelerating structures. They have studied $\mathrm{C}$-band $\mathrm{Nb}$ cavities and obtained a maximum axial field of approximately $19 \mathrm{MV} / \mathrm{m}$. They also have succeeded in accelerating a continuous electron beam from $0.08 \mathrm{MeV}$ to $0.64 \mathrm{MeV}$ in a 9-cell structure, and are designing $500 \mathrm{MHz}$ accelerating cavities for the TRISTAN electron ring.

A group of about ten people is working on the development of superconducting magnets. A model dipole for. TRISTAN is in construction. The design and the conductor used are similar to those of the FNAL energy-saver dipole. There are other superconducting magnets in various stages of construction.

In collaboration with the Japanese National Research Institute for Metals (NRIM), KEK plans to construct a 7-8 T dipole using $\mathrm{V}_{3} \mathrm{Ga}$ multifilament superconductor developed at NRIM. They will also soon try out a $7 \mathrm{~cm}$ aperture $35 \mathrm{~cm}$ long dipole using $\mathrm{Nb}_{3} \mathrm{Sn}$ conductors.

2. Institute for Nuclear Study (INS), University of Tokyo

The major accelerator project proposed for the INS is the Numatron, a heavy ion synchrotron capable of providing heavy ions up to uranium in an energy range of $0.1-1.3 \mathrm{GeV}$ per nucleon. The R\&D effort related to this construction project is concentrated on a Test Accumulation Ring for Numatron (TARN). This is a $5.06 \mathrm{~m}$ radius ring for the study and development of the injection stacking schemes and the high vacuum system. The construcfjon of TARN was completed last year. An average pressure of $2 \times 10^{-11}$ Torr was attained after 50 hours of baking at $250^{\circ} \mathrm{C}$ and $1 J 00$ hours of pumping. Injection studies started in August 1979. Simultaneous betatron and momentum stacking was successfully performed, but it has not yet been possible to make a reliable measurement of the efficiency.

The University of Tokyo group is building a $5 \mathrm{~T}, 13 \mathrm{~cm}$ aperture, $6 \mathrm{~m}$ long superconducting solenoid for the KEK muon channel. 


\section{Superconducting Work in Industry}

Several companies are engaged in the development of high field superconducting wires. Furukawa Company is developing $\mathrm{Nb}_{3} \mathrm{Sn}$ and $\mathrm{V}_{3} \mathrm{Ga}$ wires, and Sumitomo Metal Company is developing $\mathrm{Nb}_{3} \mathrm{Sn}$ wire.

C. HEP Accelerator R\&D in the USSR

Accelerator R\&D in the Soviet Union is carried out at a number of different institutions and is broad in scope. Our information about the Soviet program may be incomplete. Some of the Soviet work is reported at international conferences and published in Western journals or translations of Soviet journals.

1. Accelerator Physics

We have no information on the relative amount of accelerator running time or operations budgets devoted to accelerator physics. There is much theoretical activity concerned with all aspects of accelerator physics, most notably in stochastic and electron cooling, non-linear beam dynamics, collective phenomena and polarized beams. Most of this activity is centered at Serpukhov and Novosibirsk.

2. Advanced Technology

(a) Superconducting magnets

Much of the activity is related to the UNK project discussed below. This work is being done at Serpukhov, Leningrad, Moscow and in collaboration with Saclay. The basic design involves the use of cold bore magnets with warm iron.

(b) High power sources

Important developments in high power RF sources have been made at Novosibirsk wi.th the gyrocon development.

(c) Ion sources, heavy ion accelerators, collective accelerators

Thcre is intcrest in these topics at several institutes, in particular, Novosibirsk, Dubna, and Moscow.

3. Project-oriented R\&D

There are two major projects under serious consideration in the USSR, UNK and a high energy $\mathrm{e}^{+} \mathrm{e}^{-}$linac collider. 
(a) UNK

The main project which appears to be moving ahead is at Serpukhov, $5 \mathrm{~km}$ north of the present facilities. This is a proton-based facility employing the existing $70 \mathrm{GeV}$ synchrotron as an injector. A large tunnel $(4.5 \mathrm{~m}$ section) of circumference $19.2 \mathrm{~km}$ will house, ul timately, one conventional accelerator ring, to reach 400-700 GeV, and two superconducting accelerator rings to reach $3 \mathrm{TeV}$ for fixed-target physics and $6 \mathrm{TeV}$ for colliding beams physics $(p p)$. There is discussion of doing ep colliding beams and also discussion with Novosibirsk to perform $p \bar{p}$ in the conventional ring. The R\&D for UNK is centered at Serpukhov. Existing groups are carrying out R\&D programs, sometimes in collaboration with other institutions. For example, the superconducting magnet program is being carried out with Saclay, Leningrad, and others. The magnets will be built at Serpukhov.

(b) Linac colliders

The Novosibirsk institute is involved in a design study of linac colliders for $\mathrm{e}^{+} \mathrm{e}^{-}$in the multihundred GeV range. There does not yet seem to be a well-defined project; however, the institute is carrying out R\&D in rel ated areas, such as RF power sources, field breakdown tests in cavities, cavity design, etc. 


\section{RELATIONSHIP OF U.S. ACCELERATOR R\&D TO EFFORTS ABROAD}

Accelerator R\&D, like research in high energy physics which it supports, has a strong international flavor. The relationship of the U.S. accelerator R\&D effort to the other international programs which have been described is one of mutual support. The good communication that exists between U.S. physicists and those of the regions, particularly in western Europe, has permitted a sharing of a common pool of knowledge and techniques.

The U.S. accelerator R\&D effort has been highly successful in the application of advanced concepts to high energy physics. The accelerator R\&D efforts within other regions, particularly western Europe and the IISSR, have been of substantial benefit to the U.S. This can be seen by some recent imports: a stochastic beam cooling, invented and first developed in Europe; the initial demonstration in the USSR of beam cooling via a cold electron stream; and the development in the USSR of negative ion sources used in the U.S. for chargeexchange injection. In a technology-dominated enterprise such as accelerator $R \& D$, the overall advances require the contributions of the widest possible spectrum of talents and specialists. It is in this sense that the interchange between U.S. and foreign experts benefits both, and it is for this reason that maintenance and further cultivation of the mechanisms for exchange of ideas and information among the various regions active in HEP will yield further dividends.

A detailed, quantitative comparison between U.S. accelerator R\&D and that in the other three international regions is difficult because of differences in definition as to what constitutes accelerator R\&D, differences in accounting methods, and variations in the means of tinancing. Any such comparison should take account of the current U.S. allocation of approximately $3 / 5$ of present accelerator R\&D funding to research on superconducting magnet technology for the Energy Saver and ISABELLE.

The Subpanel has examined the relation of U.S. accelerator R\&D to that in the other regions on a qualitative basis by looking at manpower, current projects, and styles of doing R\&D. Accelerator R\&D in Japan is a modest effort compared to that in the U.S., western Europe and the USSR. Western Europe has, in comparison to the U.S., a somewhat larger theoretical and experimental effort. The number of Ph.D. physicists available for this work is larger than in the U.S. and they are well supported.

Accelerator R\&D in the USSR is recognized to be 1 arge and well-funded. Any examination of USSR accelerator R\&D must, of course, take special note of the creativity at the Institute of Nuclear Physics at Novosibirsk. This institute, under the leadership of the late G. Budker, has become a worldrecognized and admired source of original and ingenious ideas in accelerator technology. 
In summary, the Subpanel finds that vigorous and productive accelerator R\&D efforts exist abroad, that the efforts in western Europe and the USSR are larger in aggregate than in the U.S., that the se efforts are complementary in part to the U.S. approach, and that the strong international flavor is of general benefit to the field. 


\section{CRITICAL REVIEW OF U.S. ACCELERATOR R\&D EFFORTS}

\section{A. Challenges and Prospects}

As previously observed, the history of accel erator development is a remarkable record of the continuing introduction of new technologies and inventions. These innovations have yielded an exponential rise in accelerator energies through sharp reduction in unit energy costs of accelerators. The technologies we are now just bringing into practice should permit the continuation of this trend through the next generation of accelerators. As we look beyond this noxt generation, however, to the accelerators we will begin to need roughily ten years from now, it is clear that current technologies must be developed further and that entirely new inventions and technologies will be necessary.

The role of innovation was shown clearly in the Livingston chart of Figure 1. One saw there that each type of accelerator has gone through an evolutionary cycle and was then replaced by a more advanced type of instrument. This succession can also be shown graphically in unit cost terms. In Figure 8, the cost per (center-of-mass) energy unit for various proton accelerators is displayed versus the year of first operation of that accelerator. Such costs can only be approximate since each accelerator installation was made under special circumstances which affect its cost. Moreover, individual accelerators differ substantially in terms nf ancillary facilities. Cust comparisons between U.S. and foreign installations are difficult to interpret quantitatively due to the vagaries of exchange rates, combined with variations of true purchasing power of foreign currency. For all these reasons, the costs indicated in Figure 8 should be viewed only as qualitative indicators.

The dramatic decrease in unit costs has beell brought about by many factors, the most important being the introduction of new inventions and technologies by accelerator builders themselves. Crucial to these innovations has also been the general advance of modern technology in such areas as electronics, materials and manufacturing technique, vacuum, cryogenics and the like. To make the role of new accelerator technologies clear, each point in Figure 8, which corresponds to a particular acceleraton, is plotted with one or mure labels which indicate an important innovation used in that accelerat.nr. While these are by no mearis lhe only important ideas used in each of these machines, we have chosen to simplify by restricting the list to the weak-focusing principle, the strong-focusing principle, superconducting magnets, the colliding-beam technique, and beam cooling. Also of note are the ingenious uses of existing accelerators as injectors for the new accelerdtors or of adaptation of existing facilities to produce significantly higher energies. All of the above have been central to maintaining the downward cost trend. The "saturation" of a given technology is seen in the upper part of Figure 8. The unit cost for fixed technology remains about the same. The downward trend in unit costs shown by the graph is associated with the introduction of new technologies. 
INCREMENTAL COST PER BEAM ENERGY UNIT VS YEAR $M(1979) \not / \mathrm{GeV}-\mathrm{CM}$

PROTON MACHINES

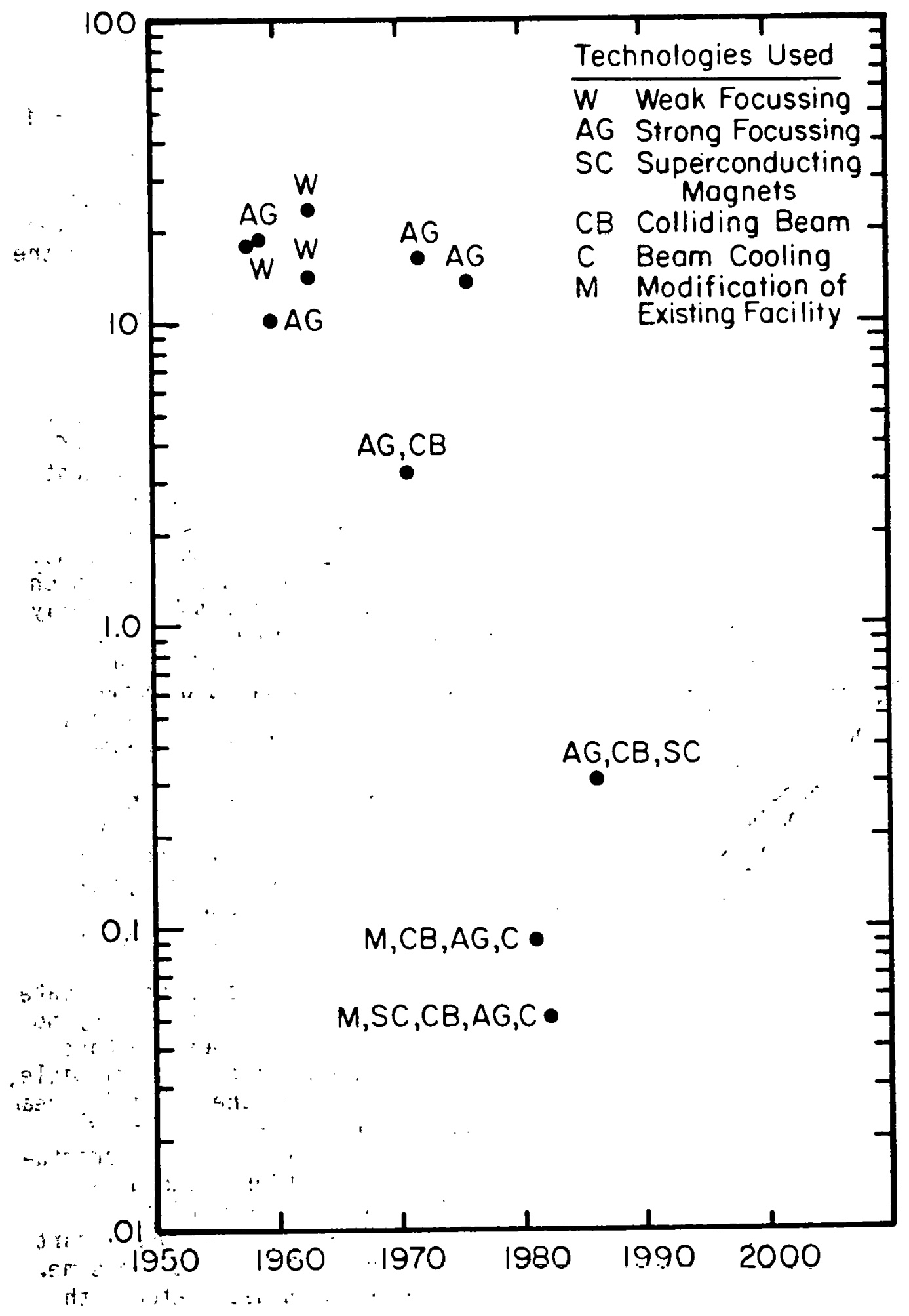

Fig. 8 
One may combine the information of Figure 8 with the Livingston plot to make projections about what one may expect for future facility costs under various circumstances. We have made a crude "fit" to the points in Figure 8. From this curve, one can obtain the unit cost of an idealized "Livingston Accelerator" for any given time. This is then multiplied by the Livingston plot CM energy at that time to yield an idealized cost for a facility of that energy. The result is shown together with the Livingston line in Figure 9. The total cost (in 1979\$) for the idealized machines, prior to 1980, is shown by the top curve. For purposes of illustration, three different cost curves are shown for the period after 1980. The middle curve is a continuation of the pre-1980 line. The top curve shows what would happen if innovation were to coase. The bullum curve shows an ideal case in which our K\&U is so successful that costs decline at the same rate that energies rise. If one makes the reasonable assumption of constant purchasing power available to the field, then the circumstanccs described by the top, "no new technology," cost curve are clearly unacceptable. In this situation, we would be unable in the future to build any new accelerators, and our elementary particle physics would soon wither away. Even if we mainlain our historical, very high, rate of progress, the middle cost line shows that, with constant purchasing power available, the number of facilities must continue to shrink if we are to provide individual facilities that, in time average, keep us on the "Livingston Line." If we are able to innovate at a rate sufficient to maintain the lower cost line, then we can meet the energy goal indicated by the "Livingston Line," while maintaining the current level of diversity of facilities.

We have tried to compile an equivalent picture for electron machines, as shown in Figures 10 and 11 . Owing to possible ambiguities in the interpretation of $C M$ enerqy for fixed-target electron machines, we have Illade our plot of unit cost, Figure 10, in terms of beam energies. All the new HEP electron machines after 1967 are colliding beam machines so that unit CM energy costs can be obtained by halving the plotted numbers for these newer machines. The electron picture is made complex by the fact that under conditions of constant technolngy, unit costs rise as the square of the CM energy due to synchrotron radiation. This is shown in the top cost curve of Figure 11, which rises at twice the slope of the "Livingston Line." The "current trend" line is based on two points only. At the 1980 point we have used established, current costs. At the 1989 point, we have used the I.EP estimates, translated into dollars by the factor $0.5 \times \mathrm{SF}=\$$. This factor, of course, cannot be very precise because of well-known uncertainties in accounting methods and currencyexchange rates. Even if the factor given is off by 50 percent, Figure 11 shows that the need for innovation in electron machines is equally, if not more, urgent than for proton machines.

While we have dwelt on unit capital costs for providing particle energy, one must not lose sight of the needs for maintaining or improving luminosities, providing extracted beams, polarized beams, etc., while 


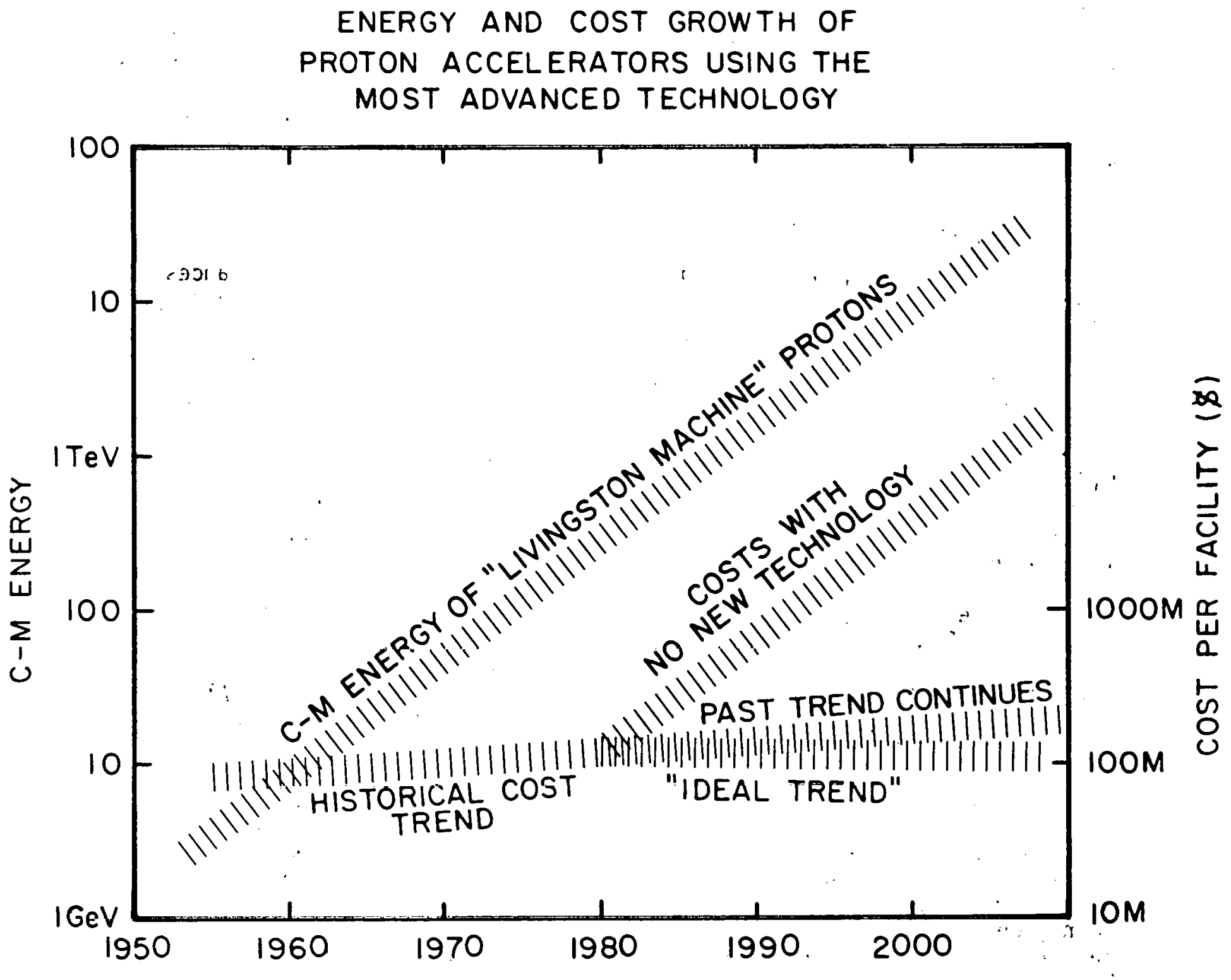

Fig. 9 
INCREMENTAL COST PER BEAM ENERGY UNIT VS YEAR $M(1979) \$ / G e V$

ELECTRON MACHINES

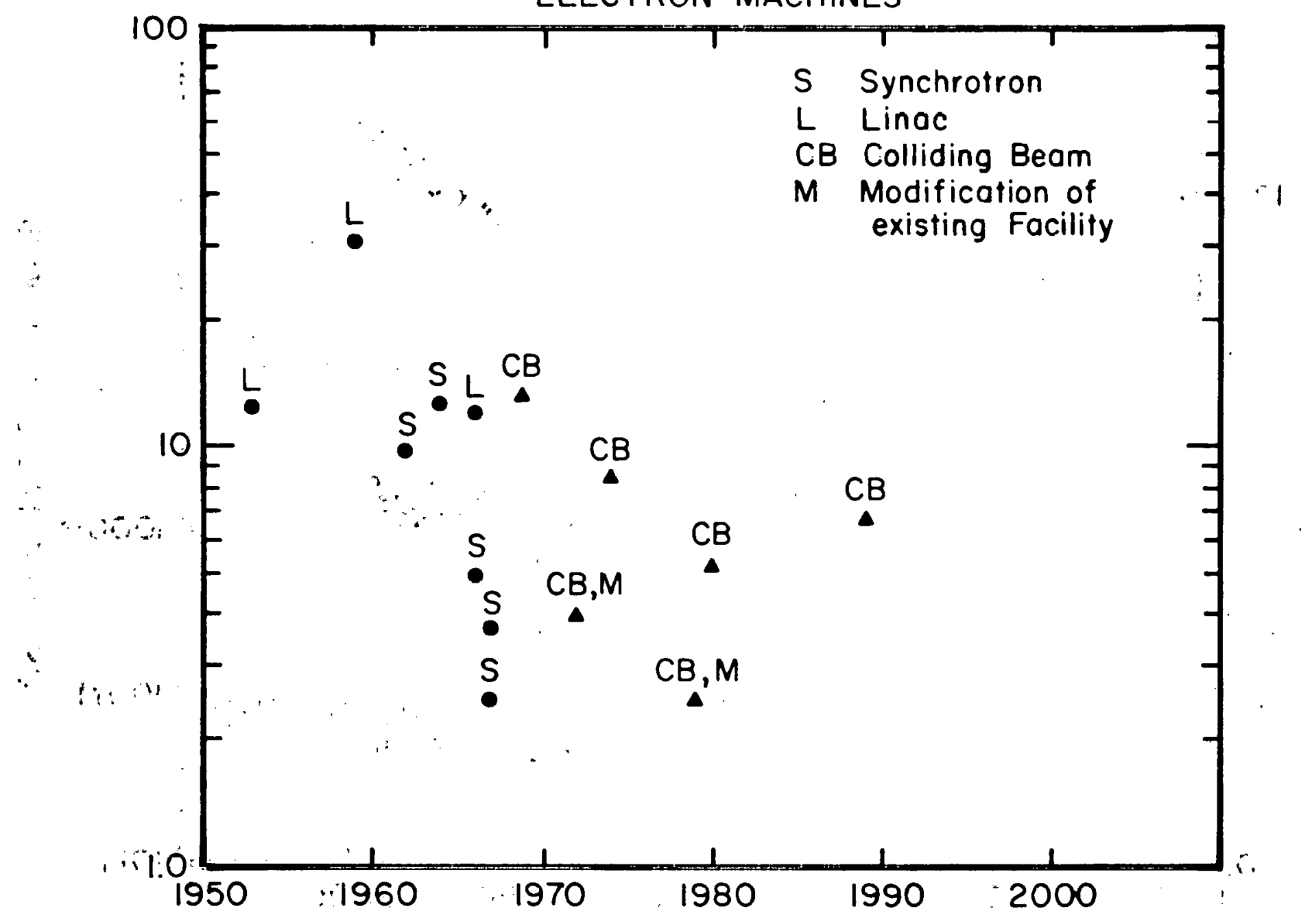

Fig. 10 
ENERGY AND COST GROWTH OF

ELECTRON ACCELERATORS USING THE

MOST ADVANCED TECHNOLOGY

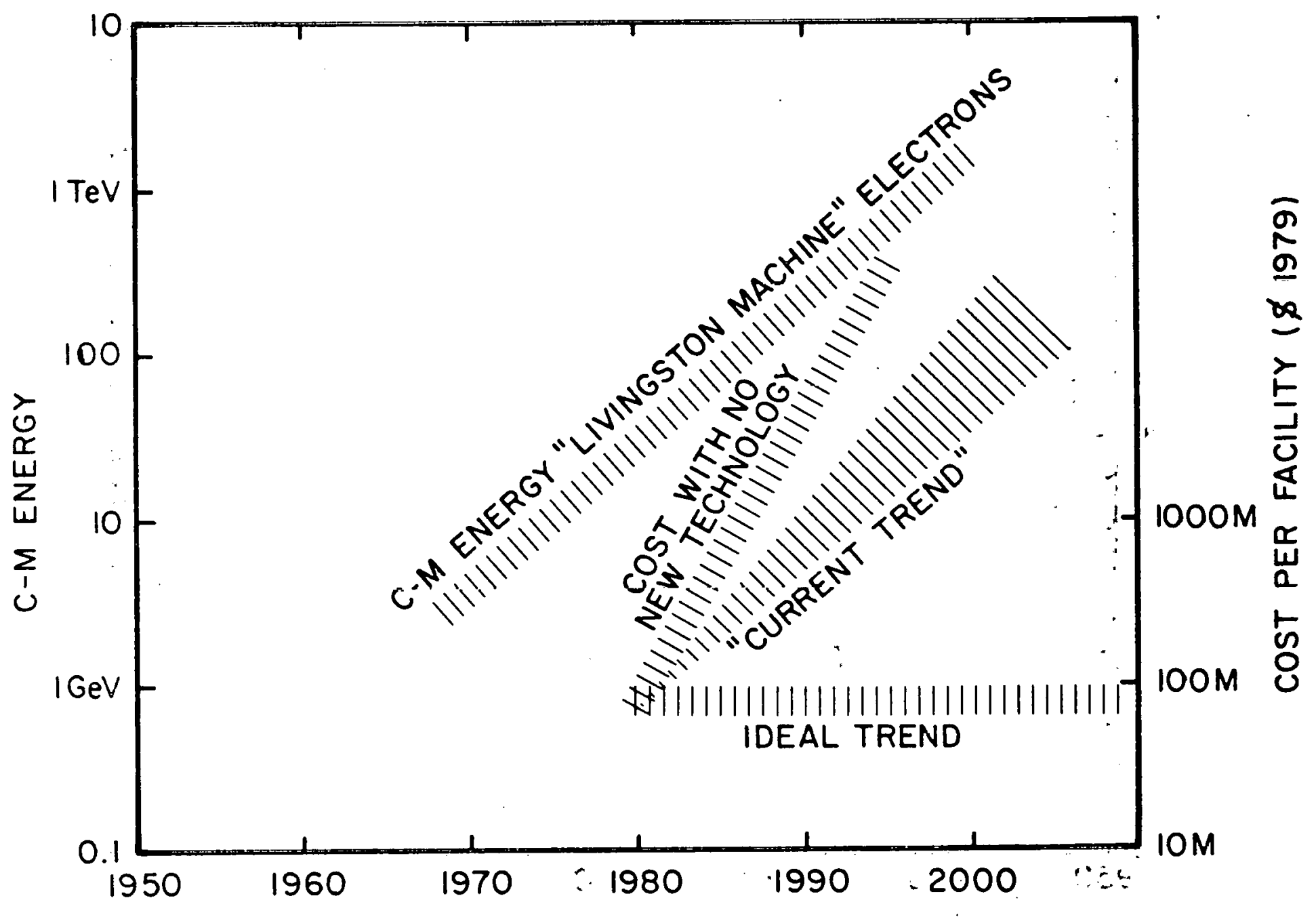

Fig. 11 
accomplishing needed energy increases. Most importantly, it will be necessary for us to apply the utmost ingenuity to the reduction of operating costs, particularly electric power costs. Currently some $10 \%$ of operating resources available to the field are used to pay for power.

These then are the challenges that we face. The prospects for further unit cost improvements are difficult to assess accurately, since one cannot anticipate invention but merely try to nurture it. As in the past, however, an important component of progress will doubtless be the further development of technologies now being used or just being brought into use. Here again assessment is unsure because one cannot anticipate invention but one can make estimates as to possible limits in unit cost improvements for current technologies. For orientation, we give two rather superficial examples.

As a first example, consider the superconducting magnets which are to provide the guide fields for the next generation of proton colliders. Superconducting magnets now in development will operate at 4 to 5 Tesla. Intensive development can perhaps lead to a 10 Tesla magnet of comparable weight and complexity, or alternatively, the cost per pound of magnets of current performance can be improved. The combination of such developments might yield unit cost improvement factors of perhaps 2-3. These figures indicate that such efforts can make important contributions to cost reduction. Yet such improvements are limited and this fact highlights again the need for qualitatively new ideas if really large increases in energy are to be achieved economically.

As a second example, one can address electron accelerator systems in a similar vein for rough assessment of potential. The original SLAC machine achieved a gradient of $7 \mathrm{MeV} /$ meter. By raising the peak power, this was increased to $11 \mathrm{MeV} /$ meter. Further conversfon will bring it to $17 \mathrm{MeV} /$ meter. Prel iminary experiments indicate that accelerator structures similar to those now in use may be able to sustain perhaps up to 100 $\mathrm{MeV} /$ meter. This high gradient can lead to reduced unit energy costs only if the costs of the required higher power RF systems become comparable with present power systems, an area where considerable work needs to be done. Superconducting RF structures also offer substantial potential. A niobium structure can support a surface magnetic field of $1.5 \mathrm{kilogauss,}$ which corresponds to an effective accelerating gradient of about 40 $\mathrm{MeV} / \mathrm{meter}$. In such a machine the refrigerator is a major component, filling a role comparable to the RF power sources for a normal pulsed linac. In a superconducting linac, the cavity costs are likely to be at least as large as those of a normal linac. Therefore, to compete with the normal linac in terms of unit engrgy cost, the losses must be very low. Operating $Q$ 's of several $\times 10^{1}$ are required to make refrigerator costs equal to or lower than those for the RF system of a corresponding normal linac. Such $Q ' s$ have been achieved in small assemblies. 
While this simplistic approach does not tell the whole story by any means, it is evident that maintaining the improvement factor of 6 per decade, implied by the ideal cost curves presented above, would require that the full potential of existing or barely existing technologies be reached on the time scale of a decade. Looking to the decades beyond, we may be quite sure that we shall need strikingly different technologies. We should be laying their foundations now.

B. Balance of Short-Range, Mid-Range, and Long-Range R\&D

These three categories form parts of a continuum. Their boundaries are somewhat arbitrary. We have defined R\&D applied to projects (RDAP) as being activities in support of operations, current construction, and proposals aimed at starts in five years or less. As noted earlier, we are currently spending about $13 \%$ of our operating resources on RDAP, the majority of this being devoted to support of the large projects, utilzing superconducting magnets, now underway. The key question about these near- and mid-tem activities is whether they are adequate to meet the construction commitments of the program. A useful answer to this question cannot be obtained without detailed review of each project in its entirety. This we have not done. We have reviewed the RDAP components of the projects in isolation from their other important components, and do not bel ieve that current RDAP expenditures are excessive.

In view of the future challenges, detailed in previous paragraphs, we do bel ieve that AARD (Advanced Accelerator R\&D) focuses primarily on the future, is receiving insufficient attention. It is to these longer range needs that the majority of our review and recommendations are addressed.

C. Technical Areas in Long-Range R\&D Now Receiving Insufficient Attention We list here four categories among long-range R\&D activities:

1. Further refinement or extension of established devices or technologies

By this we mean to include the exploration of the ultimate performance limits of the devices and technologies used in accelerators and, most importantly, thorough analysis of the devices themselves and of the accelerator systems into which they might be incorporated. Such studies are needed to reveal the important R\&D problems that need solving and to evaluate the potential unit cost improvements that may be realized through the R\&D. Likely candidates for such studies are superconducting magnets, low temperature hel ium refrigerator systems, linac structures and microwave power sources.

2. Promising methods, devices or technologies

In this category are all forms of beam-cooling methods, stochastic cooling, low energy electron beam cooling and relativistic electron beam cooling of protons. Also in this category are linear colliding 
beam methods, superconducting RF accelerators, and methods for obtaining very high energy polarized beams with selectable polarization direction both for electrons and protons.

3. Theoretical understanding

Each new accelerator that comes onl ine is plagued by unexpected effects, most often collective effects, which limit its performance. Great advances have been made. The fact that colliding beam methods have yielded outstanding physics result.s is due to the understanding and control or elimination of several important types of instability. Nevertheless, performance is stilli sharply limited by poorly understood phenomena. In the case of the non-linear bedil-beam effect, we are still forced to base our designs on semi-empirical formulae of unknown scaling'properties and upon only qualitative understanding of the phenomena. Control or amelioration of this effect would yield benefits commensurate with substantial investments in further experimental and theoretical studies of the effect using existing accelerators. Better diagnostic methods need to be developed, and considerable beam time on existing ar.selerators must be allocated if there is to be any real istic expectation of advance in this area. Similar remarks apply to intensity limits in single-beam machines and storage rings. Success in these endeavors would result in both lowering of accelerator capital costs and, through the more efficient use of accelerated beam particles, a decrease in operating costs.

4. New or unexplored accelerator principles and technologies

It is evident that there is a need for ideas and techniques which, without enormous increases in comnlexity of apparatus, will allow qual itative increases in performance. Success here is measured in GeV/meter or per kilogram of apparatus or, ultimately, in GeV/\$. One might call this parameter capital efficiency. Equally important, of course, are ideas leading to improvements in energy efficiency. obvious carndidates for attention are in short supply.

Certainly more attention and evaluation need to be focused on laser or ultra-high power $\mathrm{mm}$ wavelength-source-driven-linacs and linear collective ettects accelerators, as well as on the power sources themselves, e.y., specially designed uplical lasers, free electron lasers, cyclotron masers and the like. While it is not clear that any of the very high current, low energy collective electron beam devices now in development will have direct HEP accelerator application, they should be examined for potential in microwave generation or in beain focusing or manipulation devices.

Perhaps the most important thing at the moment is to heighten the awareness in the community that there is a real need to be met and to be open to new ideas that may emerge. 
D. Communications Within the Field and With 0ther Fields

Communications within the accelerator community are quite good but could benefit substantially from more systematic attention. This is particularly true with regard to laboratory internal reports, the most important single communications vehicle at the present time. There is a continuing need for up-to-date books and tutorial materials. The topical workshops that have come into use in recent years have shown that, if properly focused, they can serve very efficiently both as communications media and as generators of ideas.

The principal means of communication within the field are the refereed journals, laboratory internal reports, conferences, workshops and the published proceedings of these meetings as well as books and other tutorial documents. At this time the refereed journals play a relatively minor role in the overall communications network of the accelerator field. They are important for interfield communications and do help some in the dissemination of new ideas and of synthesis and codification of information about accelerator physics and technology.

The character of the field today has brought about the wide use of less formal means of recording and dissemination, primarily laboratory internal reports and preprints. Three aspects of this medium deserve comment. Errors in such internal reports and preprints remain uncorrected and therefore such media have limited reference value. Generally, specialists already in the field find it easy to get their names on distribution lists for reports from other labs. Other younger, less well-known or more peripherally connected workers do not receive the reports directly. At some laboratories, pains are taken to collect acceleratur materials sent to individuals and to display them centrally or to copy and distribute them to the appropriate intra-lab community. At other laboratories such efforts are weak or non-existent. With regard to production of such materials, some laboratories have strong traditions of reporting, judging, and disseminating their own internal accelerator work. Such activities are clearly of benefit to the 1 aboratory itself and to the comminity at large. Other laboratories do not have such a strong tradition.

The system of topical and general accelerator conferences, both national and international, works quite well and deserves the continuing support and participation of the HEP accelerator community.

Because of the relatively small size and specialized nature of the high energy accelerator field, the creation of suitable books covering introductory through advanced topics has been difficult. The few books that have been produced are widely used and have had an enormous impact on the field, both through codifying existing knowledge into a readily understood form and through the establishment of a common terminology. More books covering recent advanced are needed. 
Communication with other fields varies from strong to weak. The widespread and effective network of the IEEE as well as commercially oriented channels keep the field in rather good contact with electrical and electronic engineering. Similar, if somewhat weaker statements can be made with regard to other branches of engineering science, such as mechanical engineering, cryogenics, vacuum technology and materials, al though it is perhaps true that more effort in keeping abreast of materials technologies, particularly with regard to cryogenic behavior of metals and plastics and of advances in adhesives, may become necessary.

Of more direct relevance to accelerator physics itself is the field of plasma physics. llere lines of communication are established through persons who participate in both fields and through scientific journals. Despite such important links, most accelerator physicists are largely ignorant of the powerful methods and insights that have been developed to deal with collective phenomena by plasma specialists and vice versa. We need to find ways to educate ourselves in the language of plasma physics so that we can adapt relevant work in that field to our needs. Clearly, two-way communication would be of benefit to both fields.

E. Manpower Resources and Training

From the data available to us, presented in Section IV.E; it appears that the manpower supply is quite elastic but that the age structure of the participants in accelerator work tends to be somewhat. nlder than that of other healthy fields. The data indicate that many workers enter the field without special formal schooling in accelerator science. This means that our accelerator physicists and engineers are nearly all trained on the job. Currently, in the U.S., most of them come with backgrounds in high energy physics with a few from engineering and plasma physics. A qualitative change in this situation is not likely to come. about. What is pussible and desirable is a more systematic training effort within the institutions doing accelerator R\&D, both national laboratories and universities. This could be aided by cuilipilations of special tutorial materials, the supporting of seminar series, and the holding of summer schools aimed at persons with various levels of experience in accelerator work.

F. Decision-making Processes and Resource Allocation

As previously noted, our decision-making machinery for R\&D work is characterized by diversity. We firmly believe this to be healthy and necessary. Within the totality of present mechanisms we have the capability to foster and support an adequate long-range R\&D effort. What seems to be lacking is a slrong enough perception of need within the community. Such a perception of need is necessary for two major reasons. First, it furnishes the moral support necessary for the various actors in the decision-making chain to make the difficult decisions required in making resources available for the work. In this chain we include user groups, in-house HEP research staffs, laboratory advisory 
bodies and laboratory directorates, as well as the agencies and Congressional Committees. Second, and of equal importance, a heightened perception of need can result in a more active participation by both HEP theorists and experimentalists.

The recommendation implied by these observations, namely that we tax the short-range program for the sake of assuring the health of the field in the rather far future is, of course, very difficult to follow. For this reason, the community needs to be assured that work undertaken is purposeful and of the highest quality. 


\section{CONCLUSIONS AND RECOMMENDATIONS}

Accelerator Research and Development has always been an integral and important part of elementary particle physics research. The physics output has been paced, to a large extent, by the improvement in performance of our accelerators. In the past 50 years, equivalent beam energies available to experimenters have increased by a factor of ten million as a result of accelerator R\&D efforts. Not only performance increased exponentially but unit costs have also been reduced significantly. Even in times of decreased real spending power for the field, significant energy increases were achieved.

Despite our remarkable achievements in unit cost reductions, these reductions have not kept pace with the energy increases achieved. This has resulted in a limitation in the number and diversity of high energy accelerator facilities available to workers in the field. Further decreases in diversity would be necessary to continue frontier research were the pace of cost reduction produced by new accelerator technologies not to increase. This would have a serious negative impact on the scientific productivity of U.S. elementary particle physics. The challenge is clear: we must redouble our efforts to reduce unit costs sharply while maintaining performance improvements through full exploitation of the potential of our current accelerator technologies ${ }^{\prime} . . . \quad \therefore$ and through invention of entirely new technologies and methods.

Historically, we have spent approximately 10 percent of our operating resources on accelerator R\&D activities. This includes both R\&D applied to projects (RDAP) and long-range R\&D (AARD) which is not associated with a specific project. Today this fraction for all accelerator R\&D is $14 \%$, reflecting our heavy commitment to superconducting magnet development. The great majority of this RDAP is, as it needs to be. The Subpanel has determined that between $1 \%$ and $11 / 2 \%$ of operating resources are now devoted to AARD. If we are to meet the challenge of the tuture, more of our resources need to be devoted to this activity. We bel ieve that an appropriate level for long range R\&D would be about $4 \%$ of HEP operating resources. In arriving at this judgment we have considered both the desirable expansion of efforts now underway and the need . . for new efforts whose costs we have estimated from experience in related, accelerator research. The following table gives a reasonable example of a... program operating at the $4 \%$ level. It is meant as an illustrative example $\frac{1}{4}$ not as a specific recommendation of the Subpanel. Nevertheless, a variety of such exercises has convinced the Subpanel that a significant attack on the : long range problems requires an investment of about this level.

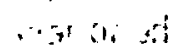


ILLUSTRATIVE FUNDING MODEL FOR ADVANCED ACCELERATOR

RESEARCH AND DEVELOPMENT (AARD)

Activity

1. R\&D on "next superconducting magnets at the approximately 10 T level generation"

\section{Current Level ( $M \$$ ) Proposed} in HEP Program Level (M\$)

$.8 \mathrm{LBL}$

1.0

$.2 \mathrm{BNL}$

2.5
Comments

These magnets will almost certainly be used in future facilities and cannot be built without substantial research into their basic physics and without development of engineering solutions to the difficult materials and structural problems invol ved.

2. R\&D on RF superconductivity
.4 SLAC

.4 Cornell
1.5

Superconducting RF cavities are a very promising approach to the problem of increasing the energies of both circular and linear el ectron accelerators. However, fundamental research remains to be done before their potential can be realized.

These studies are needed to optimize the design of any new accelerator and will be increasingly important. to future accelerator where the most economical and tightly optimized design will be a key factor in the energy and performance achieved. 
ILLUSTRATIVE FUNDING MODEL FOR ADVANCED ACCELERATOR

RESEARCH AND DEVELOPMENT (AARD)

(continued)

\section{Activity}

4. Theoretical and experimental studies of new accelerator concepts
Current Level (M\$) Proposed

in HEP Program Level (M\$)

Comments

All of our current understanding indicates that new approaches will be needed if we are to continue to move to higher energies beyond the limits we $c$ an foresee for the technologies we currently know about. Some possibilities exist and we believe several should involve a substantial experimental program at any given time. By analogy with the non-HEP accelerator projects noted in Appendix I we estimate a typical annual cost of $1 \mathrm{M \$}$ for each such undertaking.

5. Other, e.g.,

new power sources, beam man i pul at i on devices and beam diagnostic equi prient

1.0

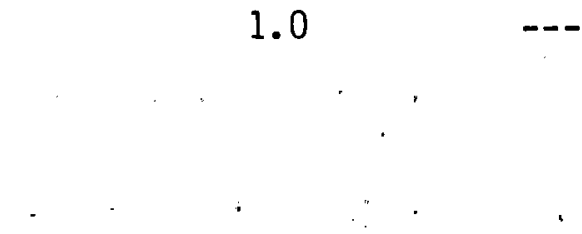$$
-2.9
$$ 
The creation of an actual augmented program should follow the established pattern of initiative and review.

It is obvious that no level of expenditure for AARD can guarantee that the historical trend in unit cost reduction can continue. Nevertheless, the Subpanel is convinced that without increased effort on AARD significant unit cost reduction is most unlikely. Thus, we conclude that increased effort on AARD is an essential investment for the community at this time.

Recommendations

1. The high energy physics community should significantly increase its support for AARD. The Subpanel estimates, from an examination of significant areas of this research, that the level for AARD in the HEP accelerator field should be increased from about $1 \%$ up to about $4 \%$ of current operating resources. This increase should be achieved within the existing administrative framework.

2. In reviewing accelerator $R \& D$, the Subpanel has identified certain specific technical areas that should be emphasized in AARD. This list is not presumed to be complete, exclusive, or to indicate relative priorities. Rather, it is intended to be an indication of the challenges of AARD and to underscore the importance of AARD to the field of high energy physics. This list of specific topics is:

(a) Development of very high field accelerator magnets and the evaluation of the practical limits of this technology. In view of the large scale of this enterprise and its uniqueness, the Subpanel recommends that this AARD effort be carried out as a collaborative effort among the laboratories having capability in this area. The Subpanel feels that this development of high-field magnets should be focused toward a specific accelerator goal.

(b) Development of liquid heliull refrigerator systems with goals of improving efficiency and reliability and providing operation at reduced temperatures.

(c) Theoretical and experimental exploration of the 1 imits to microwave linac gradients and to the peak powers that can be delivered in the $S$ - to $X$-band regions.

(d) Basic physics and device development in superconducting RF accelerators.

(e) Theoretical and experimental studies of basic accelerator phenomena, particularly the beam-beam interaction and other performance-limiting phenomena. 
(f) Search for and prel iminary development of new accel erator schemes with high performance potential such as laser accelerators or other devices using ultra-high peak power with or without collective effects.

(g) New techniques and devices for manipulating very high power and/or very high energy beams.

(h) The general problem of increasing the brightness of particle beams with emphasis on cooling high energy beams.

(i) Development of new heam diagnostic tcchniques and devices.

3. Laburdiory and University managements, aided by the agencies, should take specific measures to make participation in accelerator R\&D practical and more desirable for high energy physics theorists and experimentalists not now so engaged. This could include payments of salary for sabbatical leaves and leaves of absence; and on-the-job training through seminars, summer schools and the use of specially assembled tutorial and review materials. Al so expansion of university-labortory collaborations could be included.

4. Laboratory and University managements, aided by the agencies, should take specific measures to facilitate cross-fertilization from other fields such as Plasma Physics, Lasers and Materials Science, and other accelerator-related activities. This could include long-term visits by outstanding individuals in these fields for the purpose of working directly with laboratory staffs, giving tutorial scminars or leclures series, or participating in extended topical workshops. 
APPENDIX I

R\&D ON ACCELERATORS TO PRODUCE VERY HIGH BEAM CURRENT

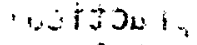

$\therefore$ in

I. INTRODUCTION

II. ... CATEGORY 1: NON-COLLECTIVE SYSTEMS

1. Ion Accelerators

2. Electron Induction Linacs

1II. CATEGORY 2: " COLLECTIVE DEVICES AND CONCEPTS

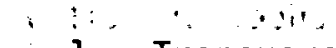

$\therefore$ 1. Transverse Focussing

2.: Longi tudinal Acceleration

$\therefore 3$. Wave Accelerators

IV. SUMMARY AND CONCLUSIONS

V. ACKNOWLEDGEMENTS

VI. SELECTED REFERENCES 


\section{INTRODUCTION}

Apart from traditional accelerator research in support of present and proposed accelerators for High Energy and Nuclear Physics, there is a considerable body of active research, largely supported by DOD funds, on accelerating devices and concepts of a novel nature. A selection of these will be reviewed below. Programmatically, these activities span a broad range of risk/benefit ratios ranging from extension of existing parameters for accelerators with well-established principles of operation to exploring speculative concepts that may in the future provide a new tool tor accelerator physicists to deploy fil constructing riuvel lypes of accelerators or expanding the capability of more traditional machines. Likewise, there is a broad spectrum of funding levels ranging from a wide variety of exploratory programs (at greater than $100 \mathrm{~K} \$ /$ Year level) through a small number of selected well-supported programs (at greater than $1 M \$(Y e a r$ level), to a single significantly large construction program (ATA at $10 \mathrm{M} \$ /$ Year level). In general, all the se programs are concerned with producing very high beam current in a pulsed mode and with the potential for later being scaled to large beam energy (joules not MeV).

It is important to note that attention in all these programs has been focussed on producing beams with kinetic energies less than about $1 \mathrm{GeV}$, and it will be seen that a rich variety of novel ideas and tools has been developed. Hitherto, there has been almost no stimulus to address, in a similar way, the applications of some of these concepts to ultra-relativistic particle beams for high energy physics applications. Given such a stimulus it could well be that the ingenuity which has been brought to bear on solving the medium energy high-current electron and proton beam problems could well in the future lead to novel advances in the art of High Energy Physics particle beams.

Broadly speaking, the programs to be discussed fall into two categories:

\section{Non-Collective Systems}

These include $r$ f linacs (for ions) and a variety of induction linacs (for electrons); here one relies on externally produced ("conventional") electric and magnetic fields for focussing and acceleration.

\section{Collective Devices and Concepts}

This category can be sub-divided into cases where the collective electric field is primarily being used:

(a) Radially $\left(E_{r}\right)$ to provide transverse focussing of intense ion beams (protbins or electrons); or

(b) Axially ( $\left.E_{2}\right)$ to provide very strong accelerating fields for ions (protons); in all such cases the radial component of the electric field, $E_{r}$, also acts to constrain the beam laterally. 
It is convenient to distinguish between these broad categories in the following way. If one ignores the charge on the particles in the accelerator beam itself then at the location of the beam we have for Category 1:

$$
\text { Div } E=0 \text {, and Curl } B=0 \quad \text { (Eq. 1) }
$$

and for Category 2:

$$
\text { Div } E=\text { rho, and, sometimes, Curl } B=j \quad \text { (Eq. 2) }
$$

In the one case the fields are produced by external conductors and have constraints set by the mechanical and surface properties of solid materials; in the other case, charged particles (electrons and/or ions) are present in free space at or near the beam location to provide fields that are not subject to the conventional boundary conditions either mechanical or electrical. It is this latter feature that has led to the very wide variety of collective effect devices that has been suggested. The other side of the coin is that of ten the electron beam used to manipulate the intense ion beams is of even higher intensity and must itself be amenable to outside control and regulation.

\section{CATEGORY 1: NON-COLLECTIVE SYSTEMS}

Devices that fall under this heading include a high-current $r f$ linac, a high current storage ring and a variety of induction linacs.

\section{Ion Accelerators}

(i) High brightness rf linac (AT-2, T. Hayward, I..ASI): The aim of this program is to produce a C.W. beam of $100 \mathrm{~mA}$ of $\mathrm{H}^{-}$ions of very small emittance ( $2 \mathrm{pi} \times 10^{-7}$ meter-radians) from an $r f$ linac accelerator. The goal of the first test-stand experiment is to accelerate the beam to 4.5 MeV. A high-quality $100 \mathrm{~mA}$ beam, i.e., of satisfactory emittance, of $\bar{H}$ ions has been successfully generated from a pulsed $(20 \mathrm{msec})$ DimovDudnikov source. A rotating version of this source which will allow for heat dissipation and permit d.c. operation is being developed.

This beam will be accelerated through a $200 \mathrm{kV}$ electrostatic column and it remains to be seen if the beam-emittance at the exit continues to be satisfactory. The beam will then be bunched and injected into an $r f$ linac operating at $400 \mathrm{MHz}$. In the first sections of the linac the emittance is expected to grow because of coupling of the transverse and longitudinal motion of the presence of collective self-fields of the intense beam. Extensive numerical simulation calculations of the emittance dilution are underway. An elecant laser diagnostic to study this coupling experimentally will sample different points within each rf bunch; the focussed laser light will convert a tiny volume of the negative ions to neutral atoms which can stream out of the machine where their angular divergence and energy spread will be measured. The transverse and 
longitudinal emittance changes can thus be mapped out cell by cell along the length of the first linac section. Enittance dilution in the front end of $r f$ linacs has been known to exist for many years, this experiment looks as if it should lead to an excellent understanding of the physica processes at work.

This program provides a good example of research in one area having spin-off into another -- in this case HEP. It was during the course of this research that the samarium-cobalt permanent magnet quadrupole, which can provide ultra-high grädients for focussing very narrow beams, was invented. It is now perceived that such quadrupoles can provide a crucial capability for handling the microscopic $e^{+}$and $e^{-}$beams at the intersection region in the proposed single-pass collider at SLAC.

(ii) High-current storage ring (AT-3, G. Lawrence, LASL): The design is underway for a storage ring to store multipie curns from the LAMPF 800 MeV proton linac. The goal is to produce an external bunched beam of 1000 amperes peak current. Numerical simulation codes have been used to understand the injection process and the beam stability for large circulating beam current. Destructive collective self-field effects must be carefully avoided at the final current level.

\section{Electron Induction Linacs}

An induction linac is made up of a succession of small pulse-power modules each time to give an energy increment to the particles at the moment of passage of the beam. In typical rf modulators the peak power capacity exceeds the C.W. power rating by perhaps a factof of ten; in pulse power devices the corresponding factor can be about $10^{5}$. Since, therefore, gigawatts of power can be supplied per module the induction linac is ideally suited (and very efficient) for accelerating very large peak beam currents (100-100,000 Amps.). A Marx generator, usually, is used to charge a pulse forming network or transmission line. The geometry of the line can be arranged so that voltage of only one polarity (accelerating) is seen by the beam. For short beam pulses a vacuum or dielectric line can be used provided the double-transit time is adequately long; for long pulses a high impedance termination (ferromagnetic toroid) is used to exclude the unwanted polarity from the beam for as long as it takes the magnetic material to saturate. (See Figure 1.)

(i) Advanced Technology Accelerator (ATA, R. Briggs, LLL): At present under construction this $50 \mathrm{MeV}$ ferrite-loaded linac is intended to deliver 10,000 amps of electrons in $50 \mathrm{nsec}$ pulses. The average repetition rate is $5 \mathrm{~Hz}$ with a burst mode capability of $1 \mathrm{kHz}$ for 5 pulses. 'Water-filled Blumleins are used for the pulse-forming lines. The 5 MeV 'gun (ETA) has been completed and has so far delivered $80 \%$ of the design current at the desired repetition rate. The development of reliable high-voltage' (250 kV) spark-gap switches to operate at 1,000 times per second was a significant technological advance. 
a)

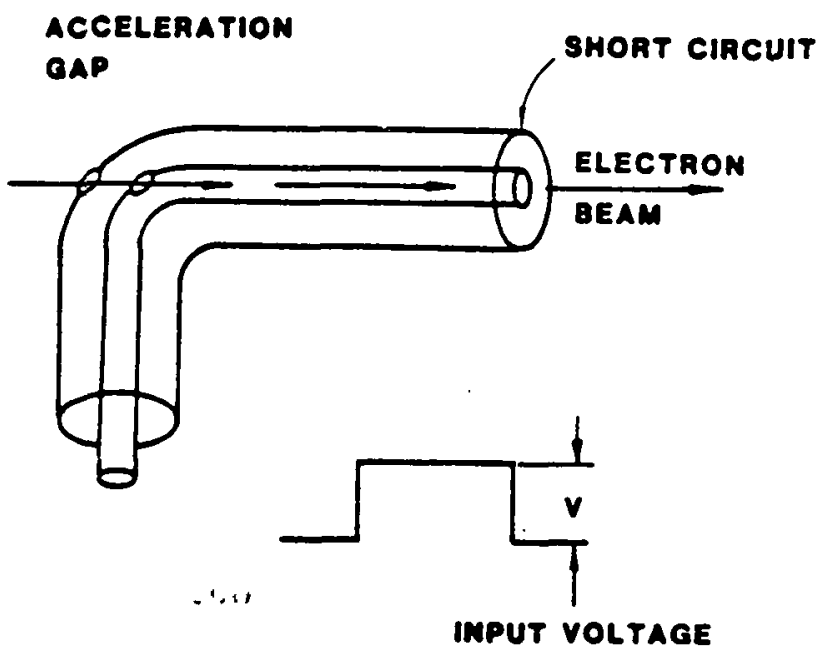

b)

\section{ACCELERATION}

OAP

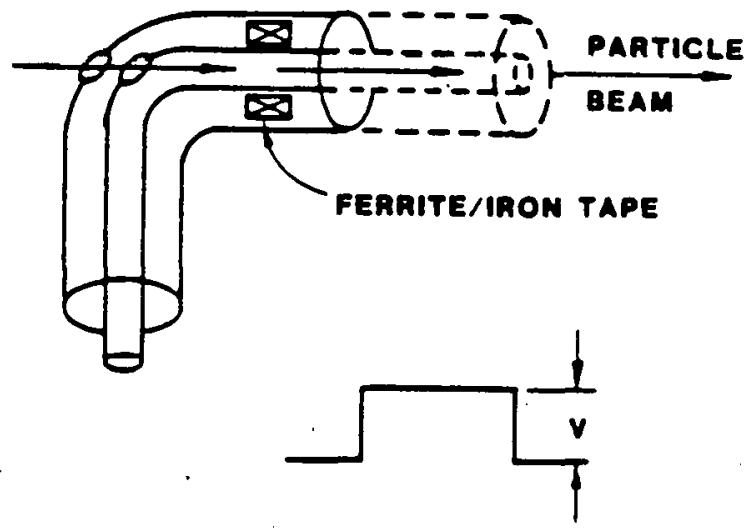

INPUT VOLTAQE c)

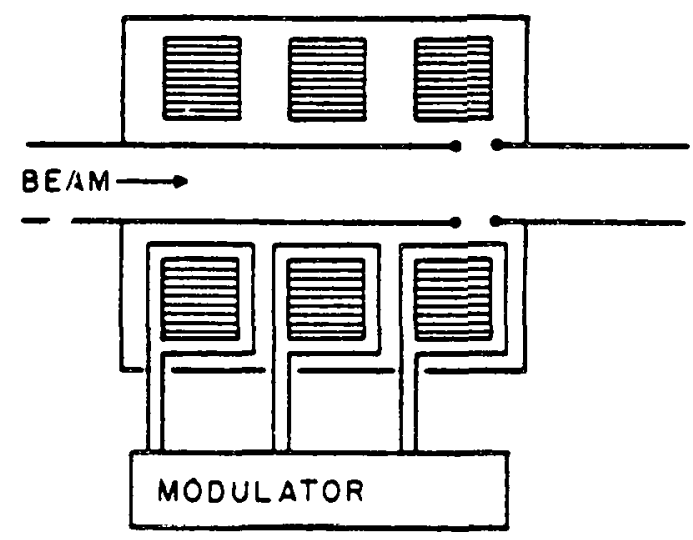

LONGITUDINAL STACKING OF ACCELERATOR CORES TO ACHIEVE VOLTAUE STEP - UP.

d) RADIAL STACKING OF ACCELERATOR CORES TO ACHIEVE VOLTAGE STEP - UP.

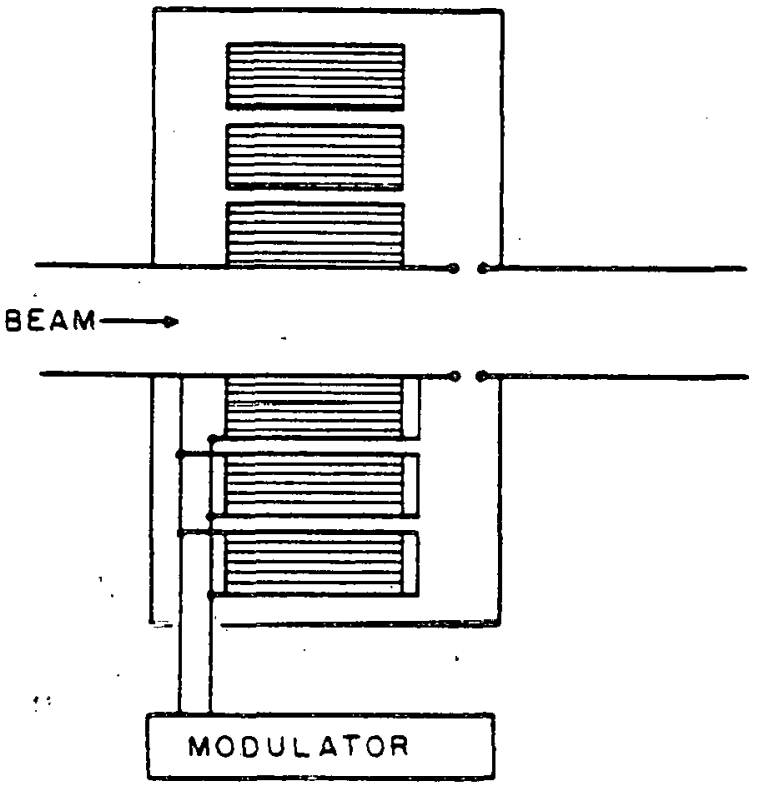

XBL $804-9241 A$

Fig. 1: Evolution of the induction linac geometry. "In (a) it can be seen that a shorted transmission 1 ine with a hollow center conductor can accelerate particles across the gap shown; the voltage disappears, however, when the inverted reflection returns, from the shorted end to the gao. For pulses much longer than. a few nanoseconds this would provide a very low accelerating gradient. In (b) ferromannetic material increases the electrical line-lenath and thus allows long pulse-jength without sacrificing oradient. Several cores driven in paralle? can provide increased aap voltage; they may be stacked axialiy (c) or radially (d). The latter was the choice for the NBS 2 -see induction lindu. 
(ii) Long pulse induction linac (S. Penner, NBS): For pulse durations much longer than 100 nsec ferrite becomes unduly expensive. The NBS program, which is now winding down, addressed the problem of using thin (one mil) inexpensive iron sheet, insulated layer to layer, as a core material suitable for a pulse duration of 2 microsec. In addition, this design included the novel feature of stacking several $(n)$ nested ferromagnetic toroids of successively larger radii. These can all be driven in parallel from a single pulse line (of voltage $V$ ) and so an accelerating voltage $\mathrm{nV}$ can be developed across a single gap. Such an arrangement leads to a reduction in the overall length of the accelerator at the expense of a more bulky transverse dimension. (See Figure $1(d)$. )

The NBS machine was operated at $018 \mathrm{MeV}$ and 1000 amps electron beam current. Experiments with this beam gave a striking demonstration that a gas-focussed beam can propagate for long distances in low pressure gas (1-30 Torr) and can even be bent through $360^{\circ}$ with dipole magnets only (no lenses, only gasfocussing) for recirculation through the accelerating cavity. This has been...". done to give a final beam energy of $1.2 \mathrm{MeV}$.

(iii) Radial line accelerator (RADLAC, B. Miller, AFWL/SA): For short pulses (about $20 \mathrm{nsec}$ ) the pulse - forming line can be a radial transmission line closed at the outer radius and, if one wishes to minimize the transverse size, it can be filled with dielectric. Such a design was first published in the USSR by Pavlovskij. The RADLAC consists of 4 such radial lines each supplying 2 MV across a two-inch gap. By use of a 2 MV pulse-power (PFN plus diode) injector the final performance is intended to be acceleration to $10 \mathrm{MeV}$ of a $50 \mathrm{kA}$ electron beam with a pulse length of $15 \mathrm{nsec}$.

If one analyzes a transmission line initially charged to voltage, $v$, which is suddenly shorted by a fast switch at one end, one finds the following voltage behavior at the open-circuit end. The voltage remains at the value $V$ for a single transit-time, tau, after switch closure; the propagating wave, $-V$ in amplitude, initiated by the short then arrives and reflects at the opencircuit with the voltage doubling to $-2 \mathrm{~V}$. The resultant voltage amplitude is $(V-2 V)=-V$ which persists for a double-transit time, 2 tau, by which time the pulse has returned from the shorted end, and is now inverted to $+V$. Thus it can be seen that, in the absence of losses, the output voltage will be a train of square pulses each 2 tau long and alternating in amplitude from $+V$ and $-V$. The only exceptional pulse is the first one which is only tau in length. For acceleration one can choose to use either the first pulse or the second if a longer pulse length is desired.

Figure 2 shows how the radial lines are arranged in the RADLAC. Each consists of a flat inner conductor flanked on either side by slightly conical outer conductors to form a tapered line of constant impedance (10 ohms). It is a "folded" geometry with both the switch and gap located at the inner radius. The oil-filled cavities are 3 meters in diameter and of fairly simple sheet-metal construction. A circular hole in the center is surrounded by a graded insulator (which provides the oil-vacuum envelope) and allows for passage of the beam. Arranged symmetrically around the cylindrical insulator are eight self-closing oil switches which fire in synchronism as the potential on the inner conductor is brought up rapidiy. During the passage of the beam no field is present on the (shorted) switch side of the line while the other side acts as an acceleratir. 


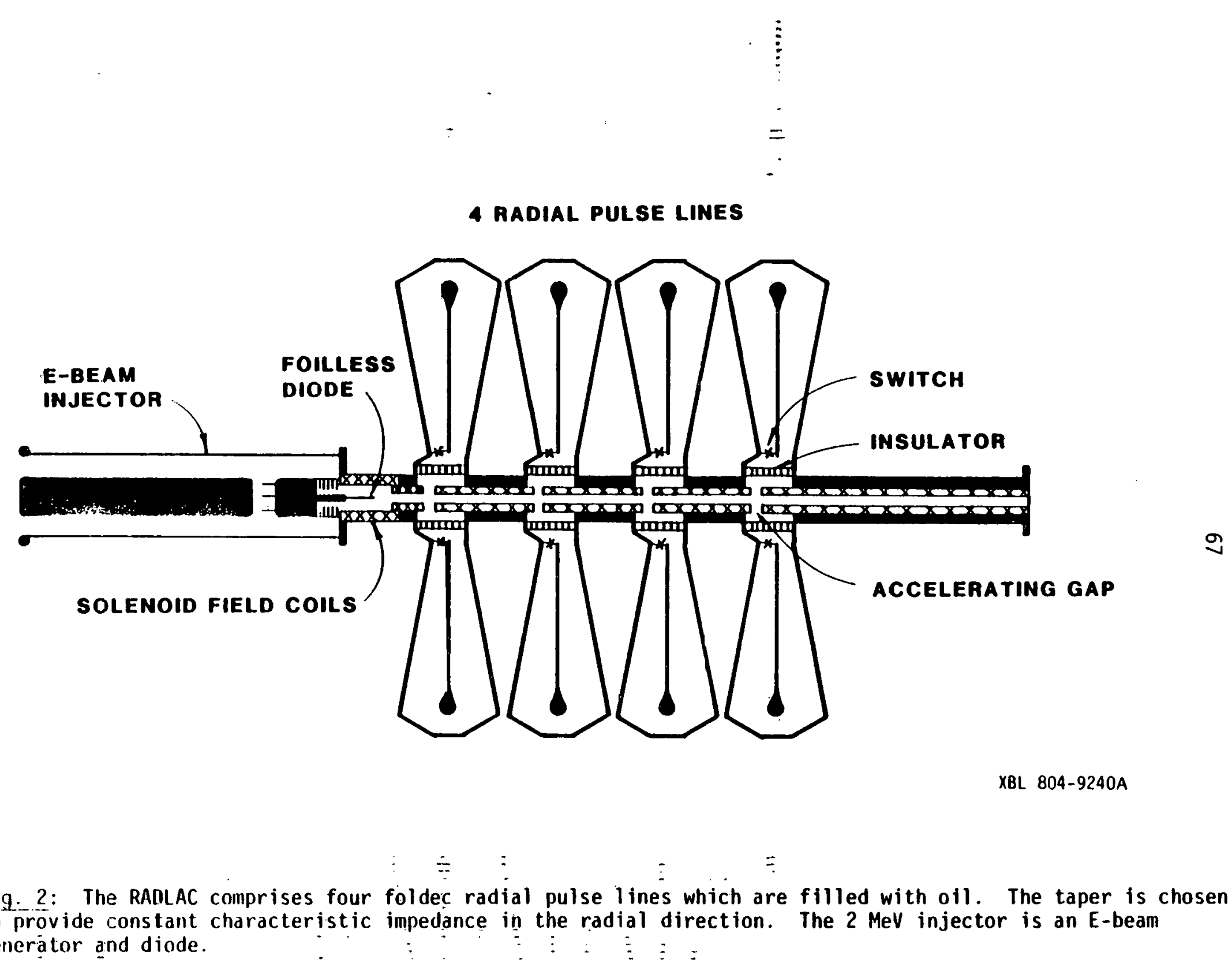
to provide constant characteristic impedance in the radial direction. The $2 \mathrm{MeV}$ injector is an E-beam generätor and diode. 
gap. Solenoid lenses provide magnetic focussing. As of early 1980, the injector and 4 cavities were operating to produce a $20 \mathrm{kA}$ beam at $9.5 \mathrm{MeV}$.

(iv) Auto-accelerator (M. Friedman, NRL): This program is an ingenious effort to exploit the high-current electron-beam technology that has been developed in the sub-10 HeV region to produce electron-beams at very much higher energies, perhaps $100 \mathrm{MeV}$. In contrast to the RADLAC geometry the cavities have their long dimension ( $\mathrm{m}$ ) in the axial, not the radial, direction (Figure 3 ). Each cavity acts as a transmission line with a double transit time, 2 tau $=6$ nsec. The mode of operation is highly novel; an intense electron beam passing through the pipe is arranged to charge the cavities with magnetic energy on a slow time scale and this energy is later extracted quickly, in a doubletransit time, to accelerate 6 -nsec pulse of electrons near the tail of the vediii pulse.

The relativistic electron "charging" beam rises linearly from zero to $I=30 \mathrm{kA}$ in a time of 800 nsec. . The beam currerl $i(t)$ acts as a eurrent source for the transmission line and instantaneously contributes a voltage, $z_{g} i(t)$, at the gap. If one follows how each such signal increment reflects back and forth along the line with inversion at the shorted end, a doubling at its first return to the open-circuit end and, in the absence of losses, repeated reflections thereafter of alternating sign, one can synthesize the voltage waveform developed across the gap. This turns out to be a linear rise to a value $Z_{0} i(2$ tau) followed by a linear fall to zero at $t=4$ tau and a repetition of this triangular form as long as the current rise continues. Thus the average value of the gap voltage is $1 / 2 \mathrm{Z}_{0} i(2 \mathrm{tau})$. We find that this is equal to $L c(t a u) d i / d t$ where $L c$ (tau) is the lumped-element (long time-scale) inductance of the coaxial cavity. The sign of this voltage is such as to provide a slight deceleration of the electron beam.

If the current rise is halted at $i(t)=I$ and the electron-beam current returned to zero the destructive reflections that keep the gap voltage at this low value are suddenly removed and it can quickly be verified that a large accelerating voltage, $Z_{0} I$, appears on the gap for a time, 2 tau. In the NRL auto-accelerator the electron-beam current is switched not to zero but to $0.2 \mathrm{I}$, so that the accelerating voltage per gap is $0.8 \mathrm{Z}_{0} \mathrm{I}$. (See Figure 3.)

What is distinctive about this device is that it circumvents two of the major problems of pulse-power accelerators -- the switches and the insulators. Since the magnetic energy release from a cavity begins just when the downward step in beam-current occurs at the yap location, the accelerator is dulomatically self-synchronized from gap to gap; jitter is eliminated since switches are not needed. Insulators at the acceleration gaps are also not required; for short pulses very high voltages ( $3 \mathrm{MV}$ ) can be achieved across just a few centimeters in vacuum. Finally, the accelerator can be designed to have relatively highefficiency from the wall-plug to the beam, perhaps in the range of $30 \%$.

In the experiments now in progress, the injector is an E-beam generator, with a transmission-line for pulse forming, which produces a $30 \mathrm{kA}, 1.5 \mathrm{MeV}$, hollow beam from a foiless diode. This beam is transported in a uniform-field solenoid 


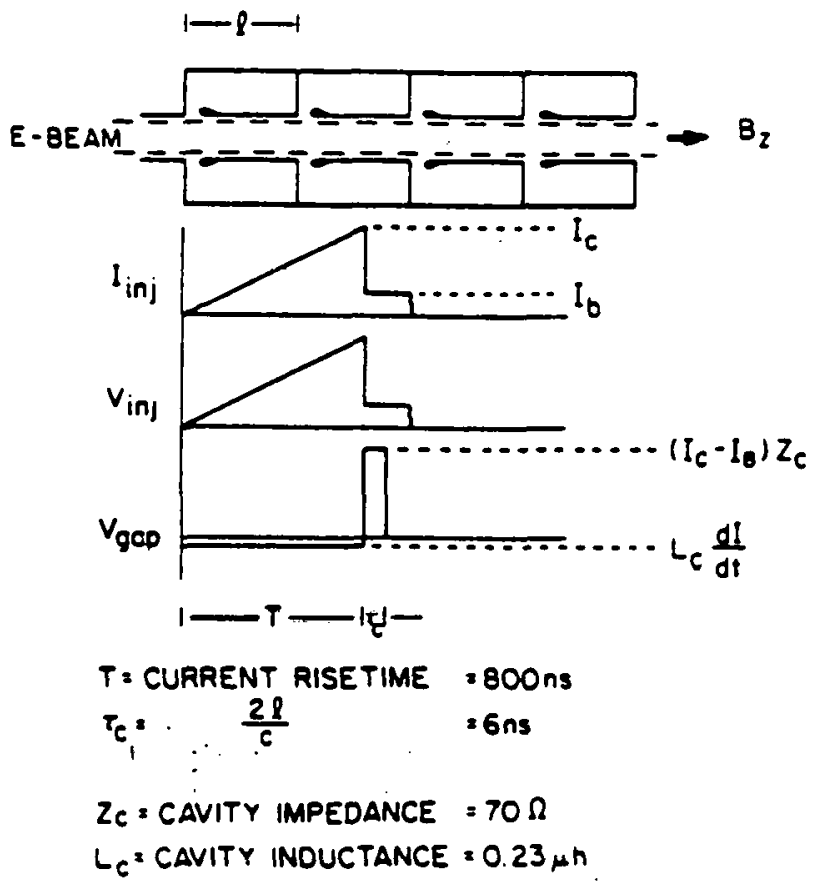

Fig. 3: The NRL autoaccelerator concept. From top to bottom the figures show: Cavity structure; injected current, $i(t)$; voltage developed across each gap showing the timeaveraged retarding voltage, $L_{c} \frac{d i}{d t}$, during the current rise, and the accelerating voltane durina the current drop.

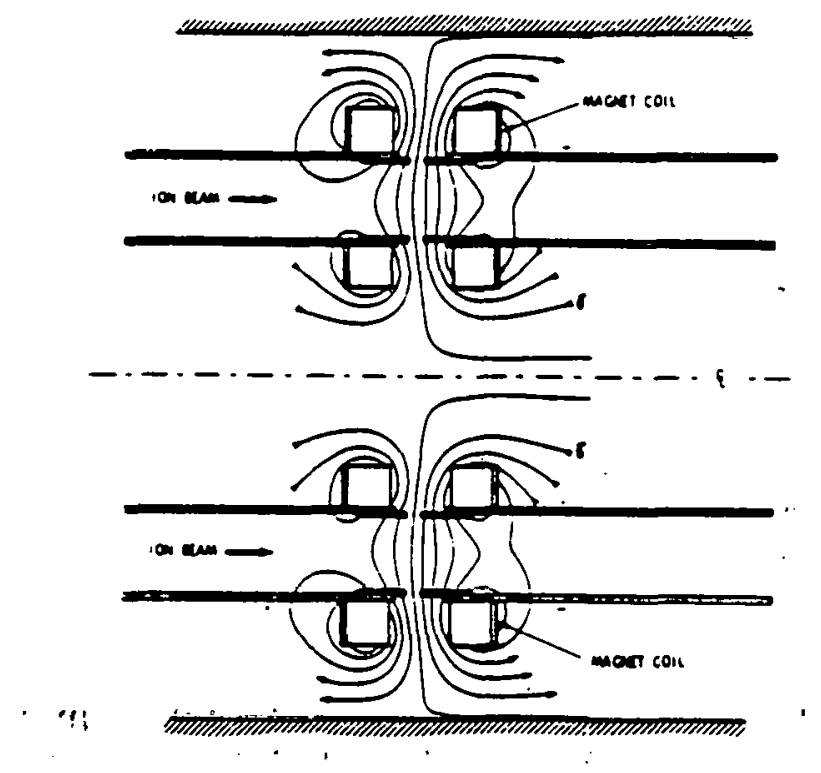

Fig. 4: The arrangement of the four field. coils. to produce the desired magnetic field in an accelerating gap of the Pulselac. Note that the ions form a hollow cylindrical beam situated in the space between the two coaxial conductors that make up a drift tube.

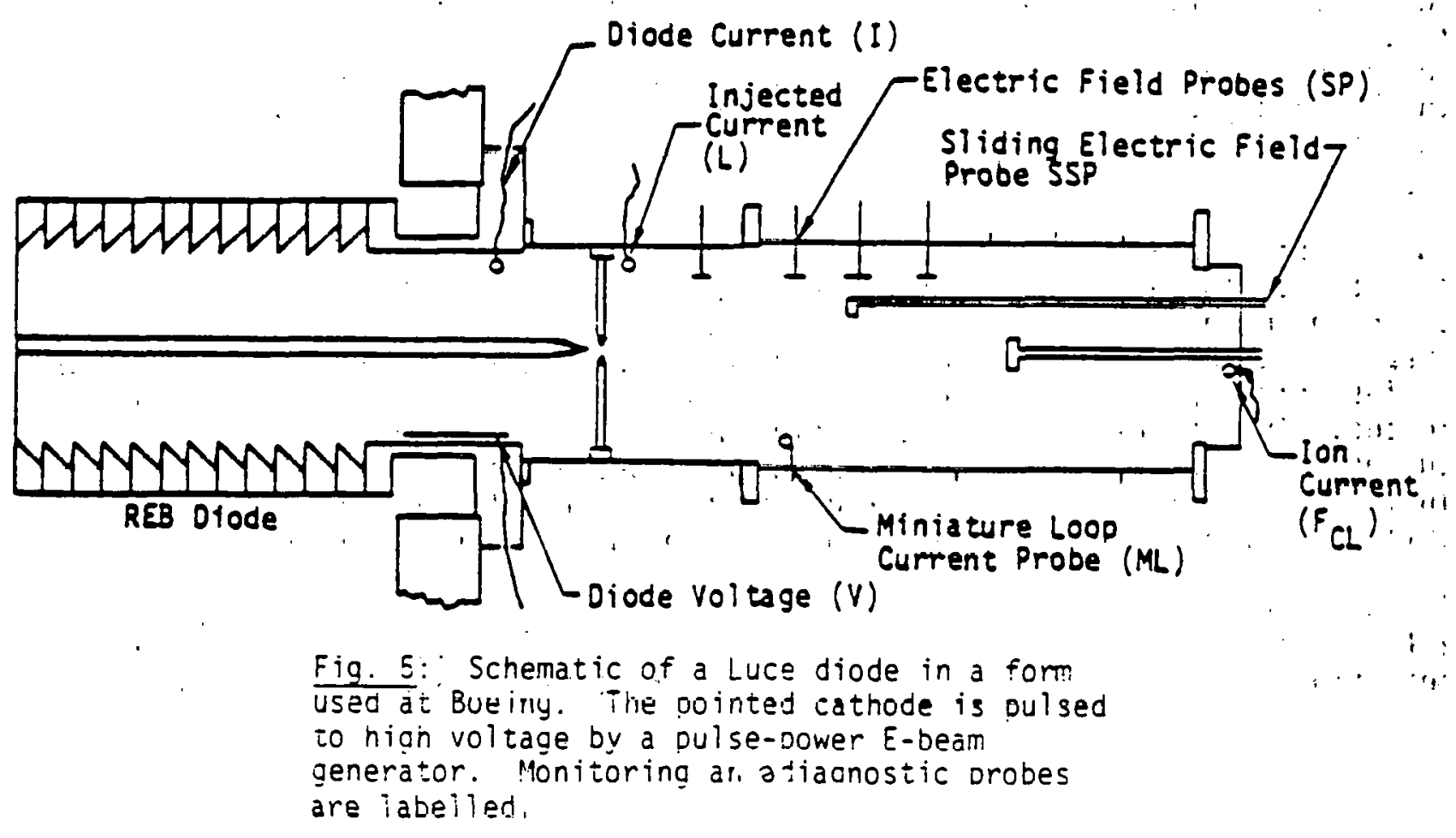


magnet (15 kG) through a sequence of coaxial cavities. Six cavities are planned for the proof-of-principle experiment; as of early 1980 two had been installed and successfully tested. Electrons were accelerated from $0.3 \mathrm{MV}$ to 3 MV with 4 kA beam current. Some "cross-talk" was encountered between the two cavities but was eliminated by reducing the Q-factor of the cavities.

It is tempting to call this device a collective accelerator in which electrons are used to accelerate other electrons but it does not comply with the spirit of the definitions in the Introduction in that the electromagnetic field occurs as an intermediary between the action of one set of electrons and the reaction of the other set. (Note, for comparison, that in a conventional rf accelerator electron-beam tubes create $r f$ fields that are coupled via wires or waveguides to cavities and thence to the beam.)

\section{CATEGORY 2: COII FC.TTVE ULVICES AND CONCEPTS}

\section{Collective Effects for Transverse Focussing}

(i) Some examples of proven utility: The transport of an ion beam from the ion-source to the Cockcroft-Walton column in a standard accelerator preinjector poses serious problems for very high intensities and for heavier ions; these arise from limitations on the strength of magnetic lenses. Several examples are now known (e.g., $60 \mathrm{~mA}$ Xe source at LBL, $150 \mathrm{~mA}$ Deuteron RTNS at LLL) where a suitably "gassy" condition of the transport line can be arranged so that ionization is caused by the head of the beam, the cold ions drift to the walls of the beam-pipe, and the electrons remain held in the space charge potential of the following parts of the beam. One can speak of "neutralization" of the self-field of the ion beam but in the spirit of the earlier definition in Eq. (2) the trapped electrons form a collective distributed lens. Instabilities can occur if there is noise in the plasma source with a period close to the ionization time or the cold-ion drift-time. Nonetheless, totally stable operation is easily achieved in which the electrons are relied upon to do as much as $97 \%$ of the focussing. For short pulses of ions, electron focussing has also been achieved at LBL by arranging for the injection of electrons from a grid; time dependent effects are still unexplored.

The universe situation has also been observed for electron beams when some gas is present; here the cold ions remain for focussing and the cold electrons are expelled. Stable operation under these conditions has, for example. uccurred in the collector tor the intense electron beam used for present cooling experiments at FNAL, and in the propagation of E-beams (LLL, NBS).

A more complicated collective mechanism enters into the stable propagation in the ballistic mode of high-current ion beams. Here the beam must be both charge-neutralized and current-neutralized, thus not alone does Div $E$ \# 0 hold but also Curl $B \# 0$ if we treat that part due to the plasma current and ignore the beam. Again instabilities (e.g., kink modes) can occur but there is a window of stability.

When an ion or electron lens created by neutralization is used as a major contributor to the focussing it is usual that the beam charge density and cold ion/electron density are close to being equal. An example of a very useful 
collective lens in which the electron density greatly exceeds the beam ion density is due to Gabor (1947). The electrons are trapped in a cylinder with an axial magnetic field and with electrostatic reflectors at the ends. In the steady state, the surface of the magnetic flux-tube concentric with the axis is an electrical equipotential and an important feature is the possibility of controlling the value of these potentials (and hence the aberrations) by properly biasing boundary rings through which the fluxsurfaces pass. Electrons can either be injected from a filament or else created by ionization of some injected gas. The lens behavior is found to be remarkably linear (BNL).

(ii) Magnetically-insulated electron-focussed ion linac (PULSELAC, S. Humphries, $S A)$ : Results to date from this program are very promising. The basic acceleration scheme is a conventional one using pulsed drift tubes to accelerate a long slug of ions. Ions are accelerated into a drift tube and when the head of the beam reaches the downstream end the voltage is removed from the drift-tube and the succeeding one switched on. Instead of using conventional focussing Humphries has arranged to inject electrons into the drift tubes to provide transverse focussing of the ion beam -- a convenient arrangement is an array of field emission points. The key feature of the scheme, however, is to prevent the electrons from crossing the accelerating gap between successive drift-tubes or they would constitute an inordinate drain on the power supply. This is accomplished by magnetic insulation whereby a magnetic field is applied in such a direction that the electrons perform magnetron orbits (with an $E \times B$ drift) but can never cross the gap and so drain the voltage generator. Obviously, fresh electrons must be injected into successive drift tubes.

Creating such a situation requires the drift tube to consist of two concentric tubes with an annular ion-beam contained between them (Figure 4). Conductors wound around the outer radius at the tips of the outer tube, and around the inner radius of the inner tube can provide a magnetic field to meet the requirement of magnetic insulation. A useful feature of this arrangement is that the $E \times B$ drift can carry the electrons around the axis again and again; thus charge-accumulation, which can be troublesome in other geometries, is avoided.

A set of plasma guns arranged in an annulus supplies about 3,000 to 4,000 amps of carbon ions for injection; a five-gap pulsed drift-tube system is now in operation that produces at its exit an impressive 3000 amps of carbon ions at an energy of $600 \mathrm{keV}$, with good emittance. These results seem to indicate that the mobile electron species can adjust its distribution in a benign way to provide focussing that is both strong and, as far as one can judge, linear.

(iii) Toroidal electron focussed ion accelerator (N. Rostoker, UCI/Maxwell): This concept is closely related to an early proposal (1956) by Budker to use an intense beam of electrons to provide a strong guide-field for protons in a circular accelerator. Several novel features are included, however, which make the idea seem attractive. The basic idea is to create a bumpy toroidal 
magnetic field, i.e., a string of mirrors that closes on itself, and to inject an intense cloud of electrons with predominantly transverse velocities (i.e., no toroidal component). The electrons form a deep potential well into which ions are injected; the ions are then accelerated by pulsing a transformer exactly as in a betatron. A design has been proposed by Maxwel! Labs that would operate with transverse collective focussing fields of $10 \mathrm{MV} / \mathrm{cm}$ and would accelerate an extremely intense proton beam to $2.6 \mathrm{GeV}$.

The key to the operation is the local trapping of the electrons in the multiplicity of mirrors. When the induced electric field is created essentially no electrons are accelerated because the loss-cone is unpopulated. This has been verified in a small experiment at UCI. Suppression of the toroidal electron current is essential to avoid taking all the energy from the generator. The design draws heavily on results from an early collective device (HIPAC) at Avco Everett in which the technique for injection of electrons with high transverse energy was developed. Also, work on HIPAC:(which was proposed by Bethe and others) succeeded in mapping out the regions of potential instabilities (diocotron, magnetron, ion-resonance) and, as a result of that work, the proposed design pays careful attention to avoiding these hazards.

A table-top experiment is underway to demonstrate proton acceleration to a few $\mathrm{MeV}$. The electron guiding field will be about $1 \mathrm{~cm}$ in minor, and $1 \mathrm{~m}$ in major diameter. So far, collective focussing fields of $500 \mathrm{kV} / \mathrm{cm}$ have been achieved. Also of interest in this program is a proposal to use a plasma gun as a collective effect extraction system.

2. Collective Effects for Longitudinal Acceleration

(a) Electron Cluster Containing lons (ERA)

This concept aims to accelerate an intense cluster of electrons which contains captured in its electrostatic well a relatively small number of ions. The lighter electrons respond rapidly to an external accelerating electric field and the protons going along for the ride thus gain energy at a rate which is enhanced by the very large factor of the mass ratio (1836 times). Since it is essential to maintain the stability of the electron cluster, Veksler proposed the creation of a relativistic electron ring which, indeed, can be stabilized. Relativistic effects for this configuration reduced the energy enhancement factor to about 40 ; even so, this would still be an exciting gain if achieved.

An extensive series of measurements was carried out at LBL, MPIPP (Garching) and Maryland, and a variety of instability limits (resistive-wal.l, negative mass, ion-resonance) identified. The potential peak accelerating rate was determined to be no more than $80 \mathrm{MeV} / \mathrm{m}$; in practice only a few MeV/m was ever demonstrated. This work is no longer pursued in the U.S. or: Germany although a very large group is continuing their program at Dubna. The Russian group has regularly accelerated protons to a few MeV and Xenon ions to over $300 \mathrm{MeV}$. 
(b) Moving Potential-Well

There is a long list of experiments in this category that have successfully produced large intensities of accelerated protons and other ions. The highest fluxes of protone usually are concentrated at a kinetic energy of about three times the eiectron beam energy although a few mechanisms have been found to give much higher energies (greater than $60 \mathrm{MeV}$ ). These experiments all employ E-beam technology in which a generator -- typically a Marx generator charging a coaxial or Blumlein line -- is connected to a pulsed E-beam diode. Fairly common parameters for the electron beams are:

$\begin{array}{ll}\text { Energy } & 1-10 \mathrm{MeV} \\ \text { Current } & 10-100 \mathrm{kA} \\ \text { Pulse Length } & \text { few } \times 100 \mathrm{nsec} .\end{array}$

The electron beem pesses through the anode foil and enters a drift-pipe which is usually (but not always) surrounded by a solenoid to provide a transverse focussing field.

Most of these experiments rely on creating a virtual cathode within the drift-pipe and arranging for it or the head of the beam to move relatively slowly down the pipe. Background ions from the walls or a gas are accelerated by this moving potential well typically to speeds of about $0.1 c$. Some experiments rely on controlling a potential well by external means.

A virtual cathode will form in a metal pipe just downstream of the anode foil if the injected beam current exceeds the so-called "limiting current" $I_{1}$. Another critical current of interest is the Alfven-Lawson current limit, which is usully significantly larger than $I_{L}$.

The trick that is used is to arrange the initial parameters such that the limiting current is below the injected E-beam current to guarantee formation of a virtual cathode by the entering portion of the beam. Next one arranges to vary one or more parameters to bring $I_{L}$ uo to the beam current and so attain propagation; if this can take place slowly enough at first background ions are trapped and accelerated.

The general features of this acceleration mechanism were elucidated in experiments at Physics International (Putman), AFWL (Miller), SA (01son). More recent work with gas gradients has been done at UCI (Mako). Experiments with dielectric walled drift pipes in which electrons escaping laterally from the virtualanode cause ions to be knocked out from the dielectric as it progresses down the pipe have been done by Pasour (NRL), Gullickson (AFOSR), Doggett (NCSU), and Greenwald (Spire). Generally these experiments led to intense fluxes of protons with energies about three times that of the electrons, although somewhat higher energies were achieved at PI with beams approaching the Alfven limit.

Much higher energy protons have been produced in a system developed by Luce (EGG). Here the anode is an insulator with an on-axis hole in it (Figure 5). While the mechanism is not well understood, it appears that a virtual cathode is formed downstream of the anode, electron bombardment 
causes flash-over of the insulator thus liberating ions that are accelerated to the virtual cathode and may pass through it to form a virtual anode. This in turn attracts the electrons and a bootstrap action persists for some distance. Proton energies up to $60 \mathrm{MeV}$ have been observed. These results have been widely confirmed at Maryland, Cornel1, AFWL, Doeing (Seattle). At the last institution Adamski has proposed a scheme in which the Luce diode can be extended to at first two and, later, several stages to obtain still higher energies.

Three schemes are under study in which a potential well is propagated at a programmed and increasing velocity by means of externally controlled elements. Rostoker (UCI) proposes to inject ions from a pulsed wall plasma to speed up the virtual cathode in a time-programmed manner. With the present equipment he hopes to achieve $10 \mathrm{MeV}$ protons and feels it can be extended to $100 \mathrm{MeV}$. In the Ionization Front Accelerator (IFA), under study by 01 son (SA), the electron beam is injected above the limiting : current into a tube containing a low-pressure working gas, which has been chosen to be cesium vapor (Figure 6). The pressure is low enough that for the duration of the beam pulse there is not enough ionization to allow the beam current to become less than the limiting current and so to propagate quickly. Arranged along the side of the tube are a series of light-pipes through which carefully timed pulses of laser light can enter to ionize the cesium. Enough ions are produced up-stream of the virtual cathode at the head of the beam to neutralize the beam space-charge in that region and reduce the potential to zero. Downstream of the beam head there is little beam present and the potential is essentially zero there, also. Thus the potential well in the beam head provides an accelerating bucket which, in fact, has quite a sharp gradient at the upstream side. By gradually advancing the region of ion creation by successive laser light pulses the will can be nudged forward at a predetermined rate to accelerate ions. In an initial set of experiments the controlled beam front motion has been demonstrated. By introducing some hydrogen gas and setting the sweep-ratc of the beam front. 01son has observed proton acceleration; the data suggest that controlled accelerating fields of $50 \mathrm{MV} / \mathrm{m}$ have been achieved.

Because it is unusual in having several features externally controllable the Collective Particle Accelerator (CPA), now under test by Friedman (NRL), is an especially interesting concept. In this, a hollow electron beam is injected below the limiting current, passed through a chopper to create a sequence of rings of charge which then enter a guide field made up of discrete short solenoids. As the train of rings passes down the rippled guide-field their radii throb alternately inward and outward. This produces an axial electric accelerating field which can be decomposed into two waves, a slow forward wave ( $v$ is less than $c$ ) and a backward wave which can be either slow or fasl, depending on the choice of parameters. The phase velocity of the accelerating wave can be controlled by varying either the inter-ring spacing or the inter-magnet spacing, and its amplitude can be varied by changing either the beam current or the magnetic field strength. Note that the mechanism proceeds by the action of discrete rings of charge 


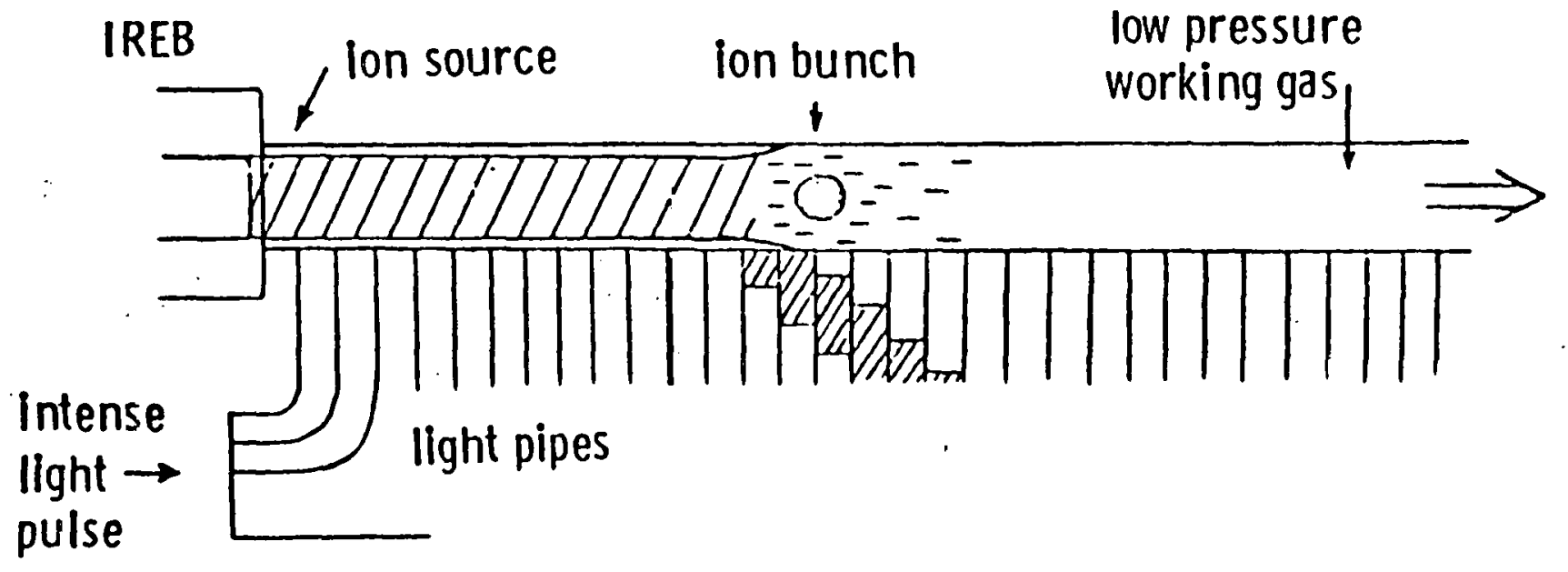

Fig. 6: Schematic of the Ionization Front Accelerator (IFA). The beam behind the bean-front has been chargeneutralized by earlier laser-ionization of the working gas; ahead of the front no significant amount of beam is present. Thus the beam-front forms a deef potential bucket which can be edged forward at a controlled rate by laser-ionization at its rear end.

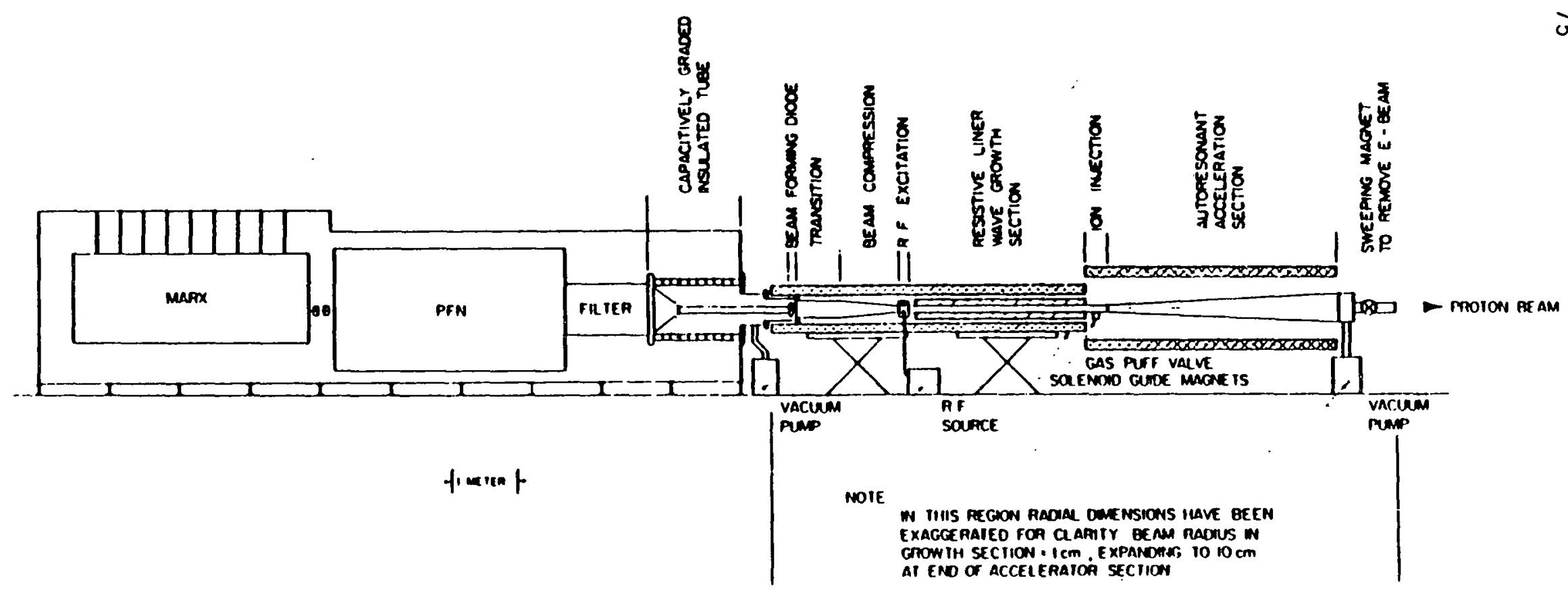

Fig. 7: Schematic of the auto-resonant accelerator (ARA). 
each of which retains its identity, and thus is quite different from the wave accelerators discussed below. It is clear that the electron rings act also to produce radial focussing of the ions. For experimental convenience, the present tests at NRL are aimed at using the backward wave to demonstrate ion acceleration. In practice, tr: forward would seem to be easier to control.

As of early 1980, experiments have proceeded to the point of generating a train of discrete rings and propagating them along the bumpy solenoid guide-field for a distance of several meters.

\section{Acceleration by Waves on Intense Relativistic Electron Beams}

Another technique for the contrulled collective acceleration of protons uses a negative-energy wave train grown on an electron beall prupagating in a vacuum and confined by an axial magnetic field. There are two experiments in progress for the last couple of years to study the pulential for application of variable phase-velocity wave-trains to collective acceleration. Both have recently succeeded in demonstrating the excitation and growth of the wanted waves and the suppression of other unwanted modes, but have not yet reached the point of injecting ions. Although each of these experiments utilizes waves of very different character -- one a cyclotron wave, the other a spacecharge wave -- both have the feature of exploiting a negative energy mode. Thus, the greater the number of ions accelerated the larger the amplitude of the accelerating field grows (until non-linear saturation occurs). Largeamplitude wave excitation creates a longitudinal modulation of the spacecharge potential, and hence a sequence of accelerating buckets, which propagate along the beam with the phase-velocity of the wave. Acceleration of the ions is achieved by arranging for the phase velocity to increase from some initial low value at injection.

(i) Auto resonant accelerator (W. Drummond, ARA, Inc.): This system utilizes a cyclotron wave (so-called lower-hybrid Doppler-shifted cyclotron mode) which has the attractive feature that the phase velocity can be made very small; thus ions can be picked up from rest by simply injecting a puff of gas at the appropriate place. Thereafter the field can be diminished in a tapered way, the phase velocity increased and the fons accelerated.

A proof-of-principle experiment is underway at Austin following extensive theoretical analysis (Figure 7). The procedure is to pass the beam (2.5 MV, $20 \mathrm{kA}$ ) through a double-helical resonant excitation section driven at $250 \mathrm{MHz}$ to excite the wave. Next comes a helical growth section which loads the wave and causes it thereby to increase in amplitude. This has now been accomplished (and incipient non-axisymmetric modes suppressed) and potential wells of about $200 \mathrm{kV}$ demonstrated, in modest magnetic fields $(2 \mathrm{kG})$. Since the phase velocity is not small the next. step will be to pass the beam into a tapered solenoid (from $2 \mathrm{kG}$ up to $20 \mathrm{kG}$ ) to slow down the wave to the point where ion pick-up is possible. Finally the flared-field accelerating section will be added. 
(ii) Space-charge wave accelerator (J. Nation, Cornell; P. Sprangle, NRL): The second system being studied for acceleration employs a negative-energy slow space-charge wave grown on the beam during its propagation through a slow-wave excitation structure. The behavior of slow space-charge waves has been long studied in the case of vacuum tubes but there are some differences for relativistic high-current beams.

It was pointed out by Sprangle et al. (NRL) that an accelerator could be built by injecting a beam of constant diameter into a pipe whose walls converged in tapered fashion towards the beam (Convergent Guide Accelerator, or CGA). Under such a circumstance the phase velocity, $v_{p h}$, can be made to increase gradually as needed. Sprangle et al. gave arguments showing that such an accelerator could give 0.5 amps of protons at $300 \mathrm{MeV}$ in a length of 15 meters.

Upon ànảiysis, however, it turns out that one cannot practically realize low values for $v_{p h}$, in contrast with the cyclotron wave case. As $v_{p h}$ goes to 0 , the beam current I goes to $I_{L}$, the limiting current. Values below $0.2 \mathrm{c}$ seem impracticable, thus such an accelerator will require an ion injector.

Experiments by Nation at Cornell in the last two years with a $250 \mathrm{kV}$ beam have shown successful growth in an iris-loaded structure of a slow wave at $1.1 \mathrm{GHz}$. The growth is rapid ( $5 \mathrm{db} /$ iris) and accelerating fields of $6 \mathrm{MV} / \mathrm{m}$ were generated. In order to study low phase velocities, he used a flared beam pipe to slow down the wave (the opposite of the CGA) and found that below $v_{\text {ph }}=0.2 \mathrm{c}$, operation was so close to the limiting current that the system was grossly erratic. "At this time he is ready to inject protons at about $20 \mathrm{MeV}$ into the wave from a Luce diode or an induction linac in order to study trapping and acceleration. Related studies are also being carried on by Sprangle et al. at NRL, who have considered a periodically loaded wall, to allow lower velocities to be achieved.

\section{SUMMARY AND CONCLUSIONS}

Although there is obviously much more work to be done in the areas of accelerator research and development discussed in this Appendix, there has, nonetheless, been very significant progress in the last few years in understanding and developing high-current accelerators and collective effect devices. Among the more important advances one can list the following:

- A new level of sophistication has been introduced into the diagnosis of emittance behavior in conventional proton $r f$ linacs. (AT-2)

- The frontiers of beam current and energy are being advanced for the electron induction linac. For years this has been a high repetition-rate pulse power device in the $1 \mathrm{kA}, 5 \mathrm{MeV}$ range: now beam currents are an order of magnitude greater and soon the energy will be increased by a like factor. (ATA)

- In the electron beam current range of $100 \mathrm{kA}$, the energy frontier is also being advanced by addition of multiply-staged pulse-power accelerating modules such as in the RADLAC and the autoaccelerator.

- Although still far from complete, understanding of neutralization techniques for providing strong transverse focussing for intense beams has advanced colisiderably (e.g., in the Pulselac and Toroidal Ion Accelerator experiments). Such techniques will undoubtedly provide a 
valuable future tool in accelerator technology.

- A new generation of experiments has begun in collective acceleration of ions. The generation of high fluxes of low energy ions by electron beams in gas-cells and related devices is now rather well understood. The accent now is on devices that can be scaled to higher ion energy ( $1 \mathrm{GeV}$ ). Tilese include multiply staged devices such as cascaded Luce diodes, wave accelerators (e.g., ARA, Cornell) and externally-controlled moving potential wells (e.g., IFA, CPA).

The broad goals of most of the accelerator R\&D addressed in this Appendix have been two-fold: the advancement of high-current relativistic electron-beam technology and the acceleration of ions from low energy. The latter is an especially difficult problem becalise of the necd to control collective fields that move very slowly initially and can be later spepded up in a programmed fashion; essentfally no thought has been given to the different question of collective acceleration of ultra-relativistic particles travelling with almost constant velocity. In fact, how this type of R\&D may in future bear on advancing the art of HEP accelerator technology is unclear, partly because it is an unexamined question but largely because by its very nature it is a long-range research field. It is complicated research still in an emergent stage; which effects and devices will pay off in the future, what their unique advantages may be, or what things are still undiscovered, are all unanswered questions.

One can perceive certain areas that could be of interest in the future of HEP accelerators as we today imagine they may be. Injectors that are compact or have some special feature (e.g., short pulse, high-intensity) are an obvious example. The scale of future $\mathrm{e}^{+} \mathrm{e}^{-}$storage rings is such that pulsepower systems might become of interest to replace the $r f$ systems. Beam extraction and manipulation will pose serious problems in TeV-class machines because of the material limitations of the septa; collective effects could be used to provide strong fields without such material problems. Direct coupling between waves on an electron beam and a relativistic proton beam is conceivable as a way to avoid losses. Pulse power technology could lead to short wavelength sources to test the laser-acceleration mechanisms. Speculation can only be vague and it is clear that much more research is lleeded before the good ideas and their applications can be put in perspective.

Because of the potential for impact on HEP technology in the future, it is clear that R\&D on novel principles of acceleration or related touls should be regarded as a valid candidate for AARD support from HEP. It is important for high-energy physicists to keep informed about developments in this field and that the HEP funding agencies be receptive to proposals for R\&D to advance our understanding in this fascinating and complicated branch of accelerator physics.

\section{ACKNOWLEDGEMENTS}

This appendix is a modified version of a report prepared with partial support from Physical Dynamics, Inc. 


\section{SELECTED REFERENCES}

1. C. 01 son and C. Schumacher, Collective Ion Acceleration, Berlin: Springer-Verlag (1979).

2. Collective Methods of Acceleration, ed. by N. Rostoker and M. Reiser. New York: Harwood (1979). 


\section{APPENDIX II}

\section{LASER DRIVEN ACCELERATORS}

I. INTRODUCTION

II. LINEAR MECHANISMS

1. Inverse Cerenkov Radiation

2. Inverse Transition Radiation

3. Inverse Synchrotron Radiation

4. Inverse Cyclotron Radiation

III. NON-LINEAR MECHANISMS

IV. THERMO-ELECTRIC MECHANISM

V. SUMMARY

VI. REFERENCES 
APPENDIX II

LASER DRIVEN ACCELERATORS

\section{INTRODUCTION}

Research with high energy elementary particles has been dominated by circular accelerators, with the one exception of the Stanford Linac. A proton synchrotron built with conventional magnets is limited to an effective gradient (top proton energy divided by circumference) of less than $100 \mathrm{MeV} / \mathrm{m}$. Because of difficulties with forces and materials, it is improbable that high field superconducting magnets will allow effective gradients much above $400 \mathrm{MeV} / \mathrm{m}$. In the case of electron synchrotrons, synchrotron radiation forces a decrease in effective gradient as the peak electron energy rises: for the largest electron synchrotron designed, the effective gradient is about $25 \mathrm{MeV} / \mathrm{m}$ or less, depending on the amount of accelerating power installed. The Stanford Linac with the SLED improvement yields about $20 \mathrm{MeV} / \mathrm{m}$. The accelerator structure could support about twice that gradient, if the $r f$ power were available.

Length of the accelerator structure is probably the most important single factor determining costs, though the nature of the equipment and the levels of peak and average power consumption are obviously very important. We note that at. LEP energies we have approached the point where the effective gradient crosses over that provided by a fairly conventional linac. Electron beams of still higher energy are almost surely provided at lower cost by linear accelerators. In the case of proton and heavier ion accelerators, it is also true that substantial increases in the effective gradient are needed if we are to maintain our pace of increase in maximum energy at roughly fixed costs. However, the goal for an attractive linear accelerator in this case must be of the order of $1 \mathrm{GeV} / \mathrm{m}$, far beyond current practice.

One parameter of such a high gradient linear accelerator is worth pointing out. If a bunch of 1017 particles is accelerated by $1 \mathrm{GeV} / \mathrm{m}$, energy is being put into the beam at a rate of $5 \times 10$ watts. The power fed into the accelerator will be greater because of finite efficiency. It is clear that the power sources must be different from those used in conventional linacs. One possibility is new types of power sources based on radiation by particle beams in material or magnetic structures: the evolution of conventional $r f$ tube development (or devices based on $r f$ storage) is mentioned in the text and described in Appendix III. Another power source which has a demonstrated capability to provide the power levels needed is lasers based on atomic systems. The power source for the acceleration mechanisms described below could be such a laser, or a free electron laser or other power source of the first class. The distinguishing element is that the wavelength must be short enough so that optical techniques may be used to concentrate the rf to high power densities. 


\section{I. LINEAR MECHANISMS}

Many schemes have been described for coupling the laser power to the particle beam. Unfortunately an electromagnetic plane wave packet acting on a free particle does not result in a net acceleration, and thus it is necessary to introduce some other bound or free charges near the particle beam. The best understood mechanisms are those that utilize the electric field in a slow wave structure excited by the laser. Their operation can be easily understood by referring to the inverse process, radiation generated by a particle passing through the structure.

\section{Inverse Cerenknov Radiation}

When a particle traverses a medium it will radiate at a given angle depending on its velocity and the index of refraction of the medium. Then if a light beam of the correct polarization were to propagate in the sense of motion of the particle and at the radiation angle, it will clearly have the correct phase relation to put energy into the motion of the particles. If the particle lies at the correct phase of the oscillating wave, it will receive a continuous acceleration. That phase relation must clearly remain correct over the length of the accelerator. This is a severe technical challenge for a high energy accelerator whose length must be of the order of a kilometer if the wavelength is in the visible or infra-red range. The medium could be a low atomic number gas, in which case the structure is relatively simple. This mechanism has been demonstrated in an experiment which achleved an accelerating gradient of $0.2 \mathrm{MeV} /$ meter. (1) This figure has since been somewhat increased. If the emittance growth due to passage through the gas is unacceptable, a solid material with a nole of diameter of the order of the wavelength of the light could be used. The effective gradient is limited by breakdown in the refraction medium, and is probably higher in the gas, perhaps approaching $1 \mathrm{GeV} / \mathrm{m}$. If longitudinal stability is necessary, adequate quadrupole focussing must be provided, to maintain an acceptance which is at best rather small.

\section{Inverse Transition Radiation}

A particle moving through a periodic structure generates transition radiation. Smith and Purcel1,(2) for example, observed light radiated by electrons passing near a plane grating. The inverse effect may be used to accelerate a particle passing near a grating illuminated by radiation at the right angle and polarization. (3) The structure must be fabricated with an accuracy of a fraction of a wavelength, but the particle beam need not pass through any material, as long as it is positioned in the near field of the grating. The advantage of this structure over the previous one is the absence of multiple scattering, perhaps more feasible methods of manufacture and alignment, and the probable higher effective gradients allowed by breakdown on reflecting surfaces rather than refracting media. Also, a damaged point on a grating can be replaced by a small motion along the grating lines. This device is a close relative of an ordinary $r f$ linac, with an unusual cavity structure and rf feed mechanism. 


\section{Inverse Synchrotron Radiation}

A static magnetic or electric field transverse to the particle direction can be used to couple a particle beam to the radiation field, as in the wigglers used in electron storage rings. They can be used to keep a particle in phase with a light beam incident along the particle beam axis and thus achieve acceleration. (4) Unfortunately, the magnetic field needed to obtain efficient coupling becomes very large as the particles become relativistic. The method might find useful application in some circumstances.

\section{Inverse Cyclotron Radiation}

In this case the magnetic field is directed along the particle direction and the phase matching is due to the cyclotron motion of the particles. (5) The magnetic field requirements for relativistic particles become still more difficult to satisfy.

\section{NON-LINEAR MECHANISMS}

There is another class of mechanisms which are driven by the power density in the rf field acting on a plasma. Since these involve the square of the electric field strength, they are known in plasma physics terminology as non-linear interactions. The attainable gradients are limited not by breakdown, but by the power available, and possible certain plasma instabilities. Another advantage is that the scale of dimensional accuracy required is set by the size of the focal spot rather than by the rf wavelength, and phase coherence is not necessary. On the other hand, very intense laser beams are needed to excite these interactions efficiently, and the analysis of an acceleration mechanism is not nearly as straightforward as for the linear mechanisms.

A simplified picture which gives a physical insight into the non-linear mechanism is shown in Figure 1. This shows a series of electrons uniformly spaced along a line, with a short wave packet of radiation incident from the left. The combined action of the electric and magnetic field on the electron produces a forward "hopping" motion of the electron, which returns to rest once during each cycle of the passing electromanetic wave. (6) After the wave packet is past, the electrons are once again at rest, but displaced along the axis. It is seen from the Figure that this phenomenon produces an enhanced concentration of electrons in the region of the wave packet. The collective field of this region of enhanced charge density can be used to accelerate and focus a bunch of beam particles. In a sufficiently dense plasma, it will excite waves of electron density and ion density.

Figure 1 depicts the situation in which the electron density is sufficiently low that the collective field does not appreciably affect the electron motion. As the density is raised, the field at the edges of the wave packet 
will oppose the hopping motion, so that the density enhancement is determined by the force which the electromagnetic wave can provide. This is called the ponderomotive force. (7)

The static field at the trailing edge of the wave packet will have the correct sign to accelerate positive particles, and over a certain region will have the correct shape to provide longitudinal stability. (8) Since the charge density is concentrated on the axis of a focal spot, there will also be strong radial focussing. The free electrons can be provided by a normal plasma of low atomic number, since the hopping motion is negligible for the positive ions, in the inverse ratio of their masses. For a relativistic particle beam, it is easy to adjust the group velocity of the wave packet to the velocity of the particles. The density must be high enough to provide sufficient charge to reach the longitudinal field available for a given laser power, but is limited by the necessary group velocity. Higher ultimate effective gradients are available at shorter wavelength. In the infra-red region, computer simulatigns have showr that gradieints of hundreds of $\mathrm{GeV} / \mathrm{m}$ are in principle possible. (9) Such linear accelerators could indeed be competitive with proton synchrotrons.

The simple picture of Figure 1 does not take into account plasma oscillations. The most prominent effect under the relevant conditions is inverse Raman scattering (9), generating waves of electron energy in the wake of the leading edge of a long wave packet. The static field due to these waves can be used for accelerating particles in the same manner as the single bunch due to a very short wave packet. The sharp leading edge, at least, is a necessary requirement for an efficient acceleration with this mechanism. The laser must have not only very high peak power, but a large bandwidth, to allow a sharp rise and preferably a very short pulse. Efficient lasers with these properties would have to be developed for this purpose, to make it possible to build a useful accelerator.

The general characteristics of the ponderomotive force have been verified by a number of experiments and energetic particles accelerated by the static collective fields generated in this way have been observed emerging from laser bombarded plasmas. (10) An experiment involving acceleration in a controlled, moving electron bunch has not yet been performed.

\section{THERMO-ELECTRIC MECHANISM}

If an intense light beam is absorbed at a solid-vacuum interface, at intensities below those where the non-linear interactions dominate, the principal mechanism is inverse bremsstrahlung. Unlike all the other mechanisms which have been described above, the energy goes into a thermal motion, primarily of the electrons. These electrons stream out of the dense plasma at the surface and into the vacuum, followed by the slowly moving plasma front. A transient, strong electrical field is thereby 
generated. It is easy to see how this could be used for generating very strong focussing fields, and it may be of interest for accelerator configurations as well.

\section{SUMMARY}

This brief description of accelerators driven by lasers or using optical focussing techniques illustrates the fact that the proposed devices span a wide range of physical effects, and the kinds of power source and necessary research are very different. Better theoretical understanding of the problems involved and the systems applications would seem the logicalifirst steps'. toward reaching the goals stated in the introduction. a

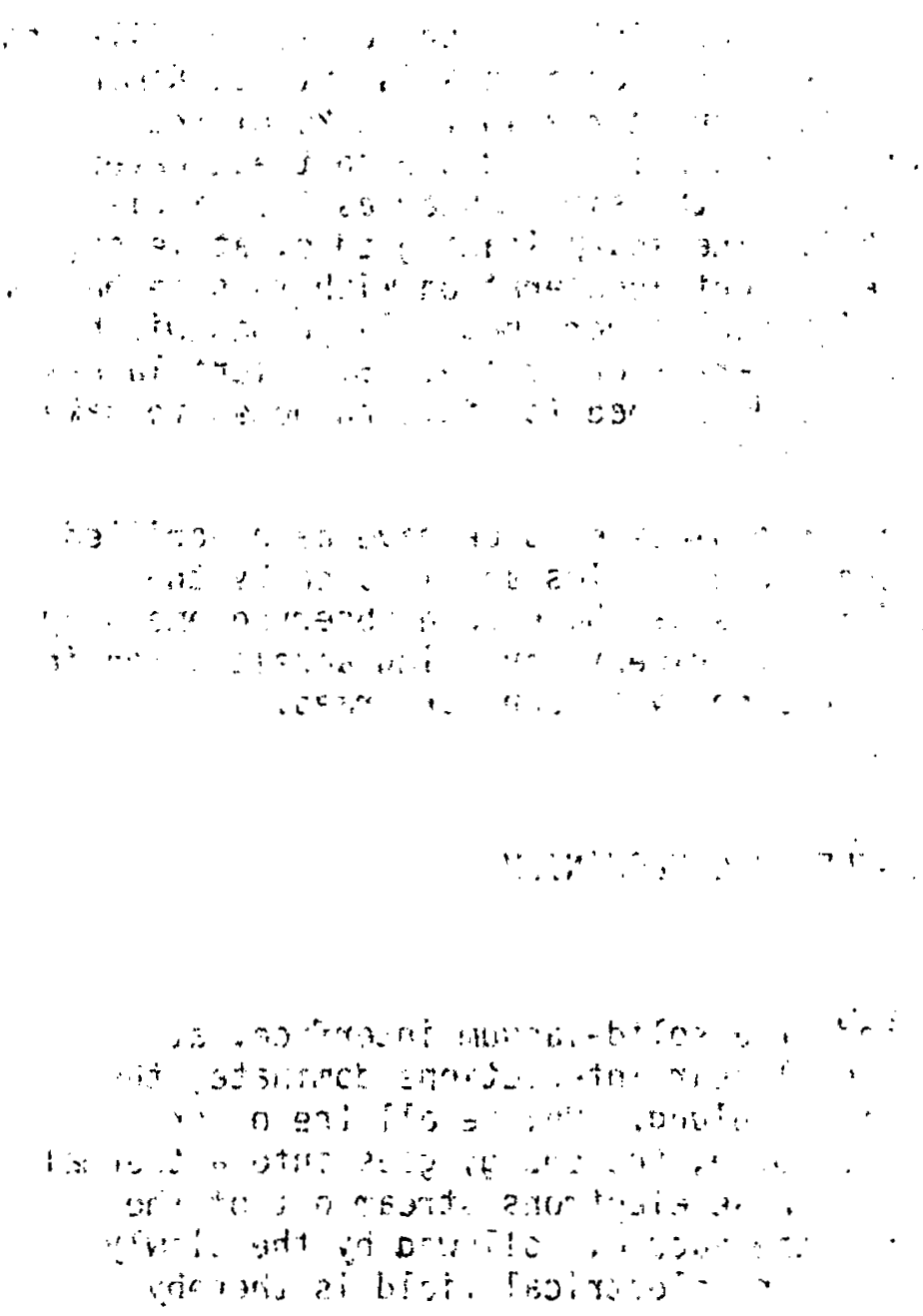




\section{REFERENCES}

1. M. Piestrup, G. Rothbart, R. Fleming and R. Pantell, J. Appl. Phys. 46, 132 (1975); J. Lawson, IEEE Trans. on Nucl. Sci., NS-26, 4217 (1979) and Report RL-75-043 (1975).

2. S. Smith and E. Purcel1, Phys. Rev. 92, 1069 (1953).

3. R. Palmer, "A Laser Driven Linac," BNL Report OG-519, March 1980, to be submitted to Particle Accelerators.

4. R. Paimer, J. Appl. Hhyś. 43, 3014 (19/2).

5. A. Kolomenskii and A. Lebedev, Dok1. Adak. Nauk USSR 145, 1259 (1962); [Sov. Phys. Doklady 7, 745 (1963), JETP 17, 179 (1963)].

6. E. McMillan, Phys. Rev. 79, 498 (1950):

7. H. Hora, "Laser Interactions and Related Plasma Phenomena," Proc. of 3rd Workshop on Laser Interactions and Related Plasma Phenomena, Rensselaer Polytechnic Institute, Troy, N.Y., 1973 (eds. H. Schwarz and H. Hora, Plenum Press, N.Y., (1974), p. 383.)

8. W. Willis, "Laser Accelerators," Proc. of 4th Workshop on Laser Interactions and Related Plasma Phenomena, 1976, p. 991; and CERN Report 75-9 (1975).

9. T. Tajima and J. Dawsun, Phys. Rev. Lett. 43, 267 (1979).

10. R. Haas, J. Holzorichter, H. Ablstrom, E. Storm and K. Manes, Opt. Commun. 18, 105 (1976). 
APPENDIX II I

NEW RF POWER SOURCES

The radiofrequency power sources now used in linear accelerators and synchrotrons, triodes and klystrons, have been brought to a high state of development after many years of effort. In the last few years new devices have begun to attract attention by virtue of their capabilities for providing higher efficiency, higher peak power, or by extending the frequency range in which very high power is available. There are still newer devices which have not been demonstrated in operation, but which appear promising on the basis of calculations.

\section{The Gyrocon}

A new microwave power source using deflection of an electron beam in a magnetic field, has attracted attention. It is mentioned in the report on work in the Soviet Union. The claim of high efficiency has beep) verified, and devices have been built to deliver very high power. Work on related devices is going on in this country.

\section{The Gyrotron}

The gyrefron was also first developed as a high power device ing the Soviet Union, although the basic principle was demonstrated here. (3) After a long and serious effort gave strikingly successful results, the development was taken up also in the U.S. This device is based on cyclotron radiation by an electron beam in a magnetic field. It is particularly effective in the millimeter region, where it has provided average powers many orders of magnitude higher than previous devices. The recent developments are intended for application in the fusion program, and are concentrated on long pulse devices. Development aimed at short pulses and very high peak powers might be interesting for accelerator applications.

\section{The Megatron}

A klystron based on multiple arrays of electrostatic quadrupole focused electron beams has recently been suggested. It has not yet been developed in practice, but it may offer another possibility of high peak powers a $t$ short wavelengths, by virtue of its small and bright electron beams, whiff maintaining large total currents through a great multiplicity of beams.

\section{The Free Electron Laser or FEL}

For which studies exist with great variations of parameters, is interesting for accelerator applications as a very high power, short pulse device with reasonably high efficiency. The version using a very high current, intense 
relativistic electron beam or IREB, to generate millimeter waves has already been demonstrated. (6) The FEL for shorter wavelengths has also been demonstrated at low power. depends on high current linear accelerator beams, and is still at the stage of design studies. Again, none of these studies has been aimed at accelerator applications.

\section{Other IREB Devices}

Other IREB devices have been demonstrated, including a version of a cyclotron radiation device, ${ }^{2}$ and the Dopplertron in which an $r f$ pulse if backscattered from an IREB in a region of rapidly falling charge density, both gaining energy and being compressed in time by a Doppler shift. (g) This shifts the frequency upward, and greatly increases the peak power. 


\section{REFEREACES}

1. G. Rutker, M. Karliner, I. Makarov, S. Morosov, 0. Nezhevenko, G. Ostreiko and I. Shekhtman, Part. Accel. 10, 41 (1979).

2. M. Zaitser, T. Pankutova, M. Petel in and Y. Flyagin, Radio Eng. Electron. Phys. 19, 103 (1974).

3. J. Hirshfie1d and J. Wachte1, Phys. Rev. Lett. 12, 533,(1964).

4. H. Jory, S. Negi i, J. Shively and R. Symons, Microwave J. 21,30 (1978).

5. A. Maschke, Internal Report (1980).

6. D. B. McDermott, T. Marshall, S. Schlesinger, R. Parker and V. Grantstein, Phys. Rev. Lett. 41, 1368 (1978).

7. D. A. Deacon, L. R. Elias, J. M. J. Madey, G. J. Ramian, H. A. Schwettman and T. I. Smith, Phys. Rev. Lett. 38, 892 (1977).

8. V. Granatstein, M. Hermdon, P. Sprangle, Y. Carmel and J. Nation, Plasma Phys. 17, 23 (1975).

9. J. Pasour, R. Parker, V. Granatstein, M. Herndon and S. Schlesinger Bul1. An. PAy. Soc. 21, 1112 (1976). 
APPENDIX IV

ACCELERATOR R\&D ON EXISTING MACHINES

Questionnaries were sent to laboratories with major HEP machines. For each machine, information was requested separately for accelerator R\&D to improve the present machine performance and for R\&D aimed at mid- and long-range goals. The responses are summarized in the following tables. 
Machine

FNAL Linac and Booster

FNAL Main Ring and Switchyard

AGS

SLAC Linac

SPEAR

CESR
Fraction of Operating

Time Devoted

to This R\&D

$3 \%$

$7 \%$

2-turn injection

Power supply chokes

Abort

Magnet improvement

Extraction instrumentation

Meson 3-way split

Septa

$5 \%$

New slow extraction

$\mathrm{H}^{-}$injection

New fast extraction

Vt jump

$3 \%$

Electron gun optics

Increase positron yield

nsec pulses from guns

SLED

Beam position monitors

Beam breakup

Polarized electron sources

$15 \%$

Beam-beam limit

Flip=flop instability

Synchrobetatron resonances

Bunch-lengthening cavity

Diagnostics

Beam gymnastics

Single beam instabilities

Intensity from linac and synchrotron
Future R\&D Topics

New "rfq" to replace first $2 \mathrm{MeV}$

\section{New extraction \\ improvements \\ Better Septa}

Better rf for linac Polarized protons

Better control system

Instrumentation and control for SPC
Wiggler magnets to increase luminosity

Beam instabilities, both single and beam-beam 
ACCELERATOR R\&D TO IMAPRONE PRESENT MACHINE PERFORAMCE - U.S. (cOAt.)

Machine

ZGS
Fraction of

Operating

Time Devoted

to This R\&D

$5 \%$
RED Topics in Past

2 or 3 Years

Future R\&D Topics

Polarized deuteron acceleration

Crossing of imperfection resonances

Polarized proton ion source developwent 
ACCELERATOR R\&D ON PRESENT MACHINES FOR LONG-RANGE GOALS - U.S.

Fraction of

Operating

Time Devoted

Machine

FNAL Main Ring and Switchyard

AGS

$1 \%$

$5 \%$ in 1980

(little until recently)

$5 \%$

$1-2 \%$
R\&D Topics in Past

2 or 3 Years

Beam loss on SC magnets

Saver injection

DO extraction

(new scheme)

Power supply ripple

Feasibility of polarized protons in AGS

Single bunch emittance measurements

Beam breakup measurements

Accelerator rf steering mea surements

Single-bunch position monitor tests

Beam-beam limit with little damping Bunch lengthening over wide range of conditions

$\mathrm{H}^{-}$polarized ion source Polarized proton storage studies
Future R\&D Topics

Simultaneous operation of Main Ring and Saver

Quenching with beam

Beam storage

Injection, abort, extraction

Better linac $r f$ technology

Non-destructive phase space monitor

Laser gun emittance mea surements

Positron target yields

Single-bunch diagnostics and control

New accelerator structure tests

Continuation of current work 
ACCELERATOR R\&D TO IMPROVE PRESENT MACHINE PERFORMANCE - NON-U.S.

Machine

CERN PS

(Linac, Booster)

CERN SPS

CERN ISR

DESY Synchrotron

DORIS

PETRA

(inc.ludes "long term" R\&D)
Fraction of

Operating

Time Devoted

to This R\&D

$6 \%$

$5 \%$

$15 \%$

$3 \%$

$5 \%$

$30 \%$
R\&D Topics in Past
2 or 3 Years

Working points (Qh, Qv), stop bands

Instabilities

Diagnostics

Resonant extraction Multi-batch injection

synchrotron 1 ight from protons

Increase of intensity

Stochastic cooling

$\bar{p}^{\prime} s$ in ISR

Beam characterislics

via Schottky noise

Overlap knockout

Microwave instabilities

Injection orbit

Feedback for head-tail instability

Beam-beall int.

Instabilities

Satellite resonances

[nergy $>5 \mathrm{GeV}$

Space charge limit on luminosity

Satellite resonance

Automatic tune regulation

Vertical instabilities
Future R\&D Topics

Beam behavior with modified densities (longitudinal and transverse)

Fast accelerating cycles

Increase of intensity

pp collider tune up

Five balches from injector

Extraction at higher intensity

Superconducting low beta $\bar{p}$ operation

Ream diagnostics Orbit correction Electronics

Mini-heta sections Wiggler

Second harmonic for bunch lengthening Cavity with higher shunt impedance Mini-beta insertions 
ACCELERATOR R\&D TO IMPROVE PRESENT MACHINE PERFORMANCE - NON-U.S. (cont.)

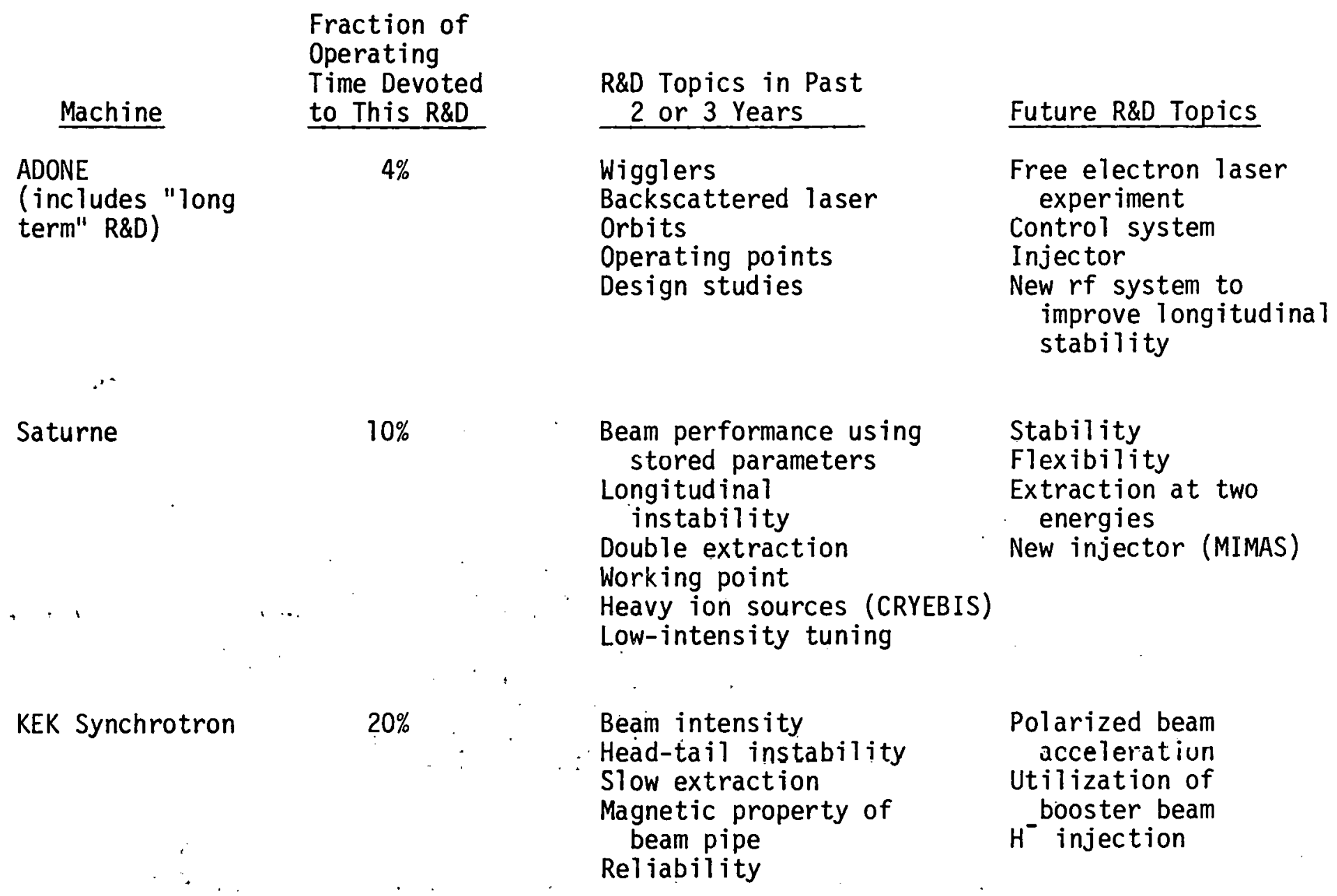


ACCELERATOR R\&D ON PRESENT MACHINES FOR LONG-RANGE GOALS - NON-U.S.

Machine

CERN PS

CERN SPS

CERN ISR

DESY Synchrotron

DORIS

KEK
Fraction of Operating Time Devoted to This R\&D

$4 \%$

$: 10 \%$

$5 \%$

$3 \%$

$3 \%$

$3 \%$
R\&D Topics in Past 2 or 3 Years

Injection and acceleration of vertically stacked booster beams

Longitudinal combination at high energy of intense bunches

Deceleration of protons for electron and stochastic cooling

Bunch lifetime

Emittance growth

New beam monitors

SC quads for low beta insertions

Cold bore

Beam-beam interaction

Bunched beam lifetime
Future R\&D Topics

$\bar{p}$ acceleration for SPS $\bar{p} p$

$\bar{p}$ deceleration for LEAR

Stochastic extraction and stochastic debunching for LEAR pp tune-up

$\mathrm{pp}$ improvement of performance

Beam dynamics

Beam-beam effects

$\overline{\mathrm{pp}}$ tune-up
High-density single bunch ácceleration

Change in optics for horizontal damping Increase injection energy Modify ejection channel to improve optics

SC rf cavity

SC magnet

SC rf cavity

U1 tra-high vacuum
SC magnet

SC rf cavity

Colliding beam machine physics 